REPRESENTATION THEORY

An Electronic Journal of the American Mathematical Society

Volume 10, Pages 254-298 (April 17, 2006)

S $1088-4165(06) 00309-8$

\title{
CHEREDNIK ALGEBRAS AND HILBERT SCHEMES IN CHARACTERISTIC $p$
}

\author{
ROMAN BEZRUKAVNIKOV, MICHAEL FINKELBERG, AND VICTOR GINZBURG, \\ WITH AN APPENDIX BY PAVEL ETINGOF
}

To David Kazhdan with admiration

\begin{abstract}
We prove a localization theorem for the type $\mathbf{A}_{n-1}$ rational Cherednik algebra $\mathrm{H}_{c}=\mathrm{H}_{1, c}\left(\mathbf{A}_{n-1}\right)$ over $\overline{\mathbb{F}}_{p}$, an algebraic closure of the finite field. In the most interesting special case where $c \in \mathbb{F}_{p}$, we construct an Azumaya algebra $\mathcal{H}_{c}$ on $\mathrm{Hilb}^{n} \mathbb{A}^{2}$, the Hilbert scheme of $n$ points in the plane, such that $\Gamma\left(\mathrm{Hilb}^{n} \mathbb{A}^{2}, \mathcal{H}_{c}\right)=\mathrm{H}_{c}$. Our localization theorem provides an equivalence between the bounded derived categories of $\mathrm{H}_{c}$-modules and sheaves of coherent $\mathcal{H}_{c}$-modules on $\mathrm{Hilb}^{n} \mathbb{A}^{2}$, respectively. Furthermore, we show that the Azumaya algebra splits on the formal neighborhood of each fiber of the Hilbert-Chow morphism. This provides a link between our results and those of Bridgeland, King and Reid, and Haiman.
\end{abstract}

\section{INTRODUCTION}

1.1. Let $c \in \mathbb{Q}$ be a rational number, and $\mathrm{H}_{1, c}\left(\mathbf{A}_{n-1}\right)$ the rational Cherednik algebra of type $\mathbf{A}_{n-1}$ with parameters $t=1$ and $c$ which has been considered in EG] (over the ground field of complex numbers).

For all primes $p \gg n$, we can reduce $c$ modulo $p$. Thus, $c$ becomes an element of the finite field $\mathbb{F}_{p}$. We let $\mathbb{k}=\mathbb{k}_{p}$ be an algebraic closure of $\mathbb{F}_{p}$, and let $\mathrm{H}_{c}:=$ $\mathrm{H}_{1, c}\left(\mathbf{A}_{n-1}, \mathbb{k}_{p}\right)$ be the Cherednik algebra, viewed as an algebra over $\mathbb{k}_{p}$. Unlike the case of characteristic zero, the algebra $\mathrm{H}_{c}$ has a large center, called the $p$-center. The spectrum of the $p$-center is isomorphic to $\left[\left(\mathbb{A}^{2}\right)^{n} / S_{n}\right]^{(1)}$, the Frobenius twist of the $n$-th symmetric power of the plane $\mathbb{A}^{2}$.

1.2. We consider Hilb $\mathbb{A}^{2}$, the Hilbert scheme (over $\mathbb{k}_{p}$ ) of $n$ points in the plane, see e.g. [Na1. There is a canonical Hilbert-Chow map $\Upsilon: \operatorname{Hilb}^{n} \mathbb{A}^{2} \rightarrow\left(\mathbb{A}^{2}\right)^{n} / S_{n}$ that induces an algebra isomorphism

$$
\Gamma\left(\operatorname{Hilb}^{n} \mathbb{A}^{2}, \mathcal{O}_{\mathrm{Hibb}^{n} \mathbb{A}^{2}}\right) \cong \mathbb{k}\left[\left(\mathbb{A}^{2}\right)^{n} / S_{n}\right] .
$$

Let Hilb ${ }^{(1)}$ denote the Frobenius twist of $\operatorname{Hilb}^{n} \mathbb{A}^{2}$, a scheme isomorphic to Hilb $^{n} \mathbb{A}^{2}$ and equipped with a canonical Frobenius morphism Fr: Hilb ${ }^{n} \mathbb{A}^{2} \rightarrow$ Hilb $^{(1)}$. We introduce an Azumaya algebra $\mathcal{H}_{c}$ on Hilb ${ }^{(1)}$ of degree $n ! \cdot p^{n}$ (recall that an Azumaya algebra has degree $r$ if each of its geometric fibers is isomorphic to the

Received by the editors May 4, 2005 and, in revised form, February 19, 2006.

2000 Mathematics Subject Classification. Primary.

(C)2006 American Mathematical Society 
algebra of $r \times r$-matrices). For all sufficiently large primes $p$, we construct a natural algebra isomorphism (a version of the Harish-Chandra isomorphism from [EG]),

$$
\Gamma\left(\operatorname{Hilb}^{(1)}, \mathcal{H}_{c}\right) \stackrel{\sim}{\longrightarrow} \mathrm{H}_{c} .
$$

The restriction of this isomorphism to the subalgebra $\Gamma\left(\mathrm{Hilb}^{(1)}, \mathcal{O}_{\text {Hilb (1) }}\right)$ yields, via (1.2.1), the above mentioned isomorphism between the algebra $\mathbb{k}\left[\left(\left(\mathbb{A}^{2}\right)^{n} / S_{n}\right)^{(1)}\right]$ and the $p$-center.

Remark 1.2.3. More generally, for any $c \in \mathbb{k}$, not necessarily an element of $\mathbb{F}_{p}$, there is an Azumaya algebra on the Calogero-Moser space with parameter $c^{p}-c$ such that an analogue of isomorphism (1.2.2) holds for the Calogero-Moser space instead of the Hilbert scheme. This case is somewhat less interesting since the Calogero-Moser space is affine while the Hilbert scheme is not.

The main idea used in the construction of isomorphism (1.2.2) is to compare Nakajima's description of $\mathrm{Hilb}^{n} \mathbb{A}^{2}$ by means of Hamiltonian reduction, see [Na1, with (a refined version, see \$5]) of the construction introduced in EG] describing the spherical subalgebra of $\mathrm{H}_{c}$ as a quantum Hamiltonian reduction of an algebra of differential operators.

1.3. We introduce the following set of rational numbers

$$
\mathbb{Q}^{\text {good }}=\left\{c \in \mathbb{Q} \mid c \geq 0 \& c \notin \frac{1}{2}+\mathbb{Z}\right\} .
$$

One of our main results (Theorem 7.3 .2 ) reads

Theorem 1.3.2. Fix $c \in \mathbb{Q}^{\text {good }}$. Then, there exists a constant $d=d(c)$ such that for all primes $p>d(c)$, the functor $\mathrm{R} \Gamma: D^{b}\left(\mathcal{H}_{c}-\mathrm{Mod}\right) \rightarrow D^{b}\left(\mathrm{H}_{c}\right.$-Mod $)$ is a triangulated equivalence between the bounded derived categories of sheaves of coherent $\mathcal{H}_{c}$-modules and finitely generated $\mathrm{H}_{c}$-modules, respectively, whose inverse is the localisation functor $M \mapsto \mathcal{H}_{c} \stackrel{L}{\otimes} \mathrm{H}_{c} M$.

Moreover, we have $H^{i}\left(\operatorname{Hilb}^{(1)}, \mathcal{H}_{c}\right)=0, \forall i>0$.

1.4. Now, fix $\xi \in\left[\left(\mathbb{A}^{2}\right)^{n} / S_{n}\right]^{(1)}$, a point in the Frobenius twist of $\left(\mathbb{A}^{2}\right)^{n} / S_{n}$. We write $\operatorname{Hilb}_{\xi}^{(1)}=\Upsilon^{-1}(\xi)$ for the corresponding fiber of the Frobenius twist of the Hilbert-Chow map, and let $\widehat{\operatorname{Hilb}}_{\xi}^{(1)}=\widehat{\Upsilon-1(\xi)}$ denote its formal neighborhood, the completion of $\mathrm{Hilb}^{(1)}$ along the subscheme $\mathrm{Hilb}_{\xi}^{(1)}$.

The theorem below, based on a similar result in BK, says that the Azumaya algebra $\mathcal{H}_{c}$ splits on the formal neighborhood of each fiber of the Hilbert-Chow map, that is, we have the following result (see Theorem 7.4.1)

Theorem 1.4.1. For each $\xi \in\left[\left(\mathbb{A}^{2}\right)^{n} / S_{n}\right]^{(1)}$, there exists a vector bundle $\mathcal{V}_{c, \xi}$ on $\widehat{\mathrm{Hilb}}_{\xi}^{(1)}$ such that one has

$$
\left.\mathcal{H}_{c}\right|_{\widehat{\operatorname{Hilb}}_{\xi}^{(1)}} \cong \mathcal{E} n d \mathcal{V}_{c, \xi}
$$

Remark 1.4.2. The splitting bundle is not unique; it is only determined up to twisting by an invertible sheaf.

Given $\xi$ as above, let $\mathfrak{m}_{\xi}$ be the corresponding maximal ideal in the $p$-center of $\mathrm{H}_{c}$. Let $\widehat{\mathrm{H}}_{c, \xi}$, resp. $\widehat{\mathcal{H}}_{c, \xi}=\left.\mathcal{H}_{c}\right|_{\widehat{\operatorname{Hilb}}_{\xi}^{(1)}}$, be the $\mathfrak{m}_{\xi}$-adic completion of $\mathrm{H}_{c}$, resp. of $\mathcal{H}_{c}$. 
We write $D^{b}\left(\widehat{\mathrm{H}}_{c, \xi^{-}}\right.$Mod $)$, resp. $D^{b}\left(\widehat{\mathcal{H}}_{c, \xi^{-}}\right.$Mod $)$, for the bounded derived category of finitely-generated complete topological $\widehat{\mathrm{H}}_{c, \xi}$-modules, resp. $\widehat{\mathcal{H}}_{c, \xi}$-modules. On the other hand, let $D^{b}\left(\operatorname{Coh}\left(\widehat{\operatorname{Hilb}}_{\xi}^{(1)}\right)\right)$ be the bounded derived category of coherent sheaves on the formal scheme $\widehat{\operatorname{Hilb}}_{\xi}^{(1)}$.

Fix $c \in \mathbb{Q}^{\text {good }}$. Then, for all primes $p>d(c)$, Theorems 1.3 .2 and 1.4 .1 imply the following.

Corollary 1.4.3. The category $D^{b}\left(\widehat{\mathrm{H}}_{c, \xi}\right.$-Mod $)$ is equivalent to $D^{b}\left(\operatorname{Coh}\left(\widehat{\operatorname{Hilb}}_{\xi}^{(1)}\right)\right)$.

1.5. Let $\mathbb{k}\left[S_{n}\right]$ denote the group algebra of the symmetric group on $n$ letters. Write $\operatorname{Irr}\left(S_{n}\right)$ for the set of isomorphism classes of simple $\mathbb{k}\left[S_{n}\right]$-modules. This set is labelled by partitions of $n$, since by our assumptions char $\mathbb{k}>n$. In particular, we have the trivial 1-dimensional representation triv, and the sign representation sign.

Let $\overline{\mathrm{H}}:=\mathbb{k}\left[\left[\mathbb{A}^{2 n}\right]\right] \# S_{n}$ be the cross-product of $S_{n}$ with $\mathbb{k}\left[\left[\mathbb{A}^{2 n}\right]\right]$, the algebra of formal power series in $2 n$ variables acted on by $S_{n}$ in a natural way. We consider $D^{b}(\overline{\mathrm{H}}-\mathrm{Mod})$, the bounded derived category of (finitely-generated) complete topological $\overline{\mathrm{H}}$-modules.

Given a simple $\mathbb{k}\left[S_{n}\right]$-module $\tau$, write $\tau_{\overline{\mathrm{H}}}$ for the corresponding $\overline{\mathrm{H}}$-module obtained by pullback via the natural projection $\overline{\mathrm{H}}=\mathbb{k}\left[\left[\mathbb{A}^{2 n}\right]\right] \# S_{n} \rightarrow \mathbb{k}\left[S_{n}\right], f \rtimes w \mapsto f(0) \cdot w$. Similarly, let $L_{\tau}$ denote the corresponding simple highest weight $\mathrm{H}_{c}$-module, the unique simple quotient of the standard $\mathrm{H}_{c}$-module associated with $\tau$; see [DO], BEG.

The results of Bridgeland, King and Reid [BKR] and Haiman $[\mathrm{H}]$ provide an equivalence of categories

BKR : $D^{b}\left(\operatorname{Coh}\left(\operatorname{Hilb}^{n} \mathbb{A}^{2}\right)\right) \stackrel{\sim}{\longrightarrow} D^{b}\left(\mathbb{k}\left[\mathbb{A}^{2 n}\right] \# S_{n^{-}} \operatorname{Mod}\right), \quad \mathcal{F} \mapsto \mathrm{R} \Gamma\left(\operatorname{Hilb}^{n} \mathbb{A}^{2}, \stackrel{\mathcal{P}}{\otimes} \mathscr{\otimes}\right)$, where $\mathcal{P}$ denotes the Procesi bundle, the "unusual" tautological rank $n$ ! vector bundle on $\mathrm{Hilb}^{n} \mathbb{A}^{2}$ considered in $[\mathrm{H}]$. Restricting this equivalence to the completion of the zero fiber of the Hilbert-Chow map, and using Corollary 1.4.3 one obtains the following composite equivalence:

$$
D^{b}\left(\widehat{\mathrm{H}}_{c, 0^{-}} \mathrm{Mod}\right) \underset{\sim}{\stackrel{\text { Corollary } 1.4 .3}{\sim}} D^{b}\left(\operatorname{Coh}\left(\widehat{\operatorname{Hilb}}_{0}^{(1)}\right)\right) \underset{\sim}{\stackrel{\text { BKR }}{\sim}} D^{b}(\overline{\mathrm{H}}-\mathrm{Mod}) .
$$

We recall that the equivalence of Corollary 1.4.3 involves a choice of splitting bundle $\mathcal{V}_{c, 0}$; cf. Remark 1.4.2. This choice may be specified by the following.

Conjecture 1.5.2. Fix a nonnegative $c \in \mathbb{Q}^{\text {good }}$. Then, for all $p \gg 0$, we have:

(i) One can choose the splitting bundle $\mathcal{V}_{c, 0}$ in such a way that $\Gamma\left(\operatorname{Hilb}_{0}^{(1)}, \mathcal{V}_{c, 0}(-1)\right)=L_{\text {sign }}$.

(ii) With this choice of $\mathcal{V}_{c, 0}$, the composite equivalence in (1.5.1) preserves the natural $t$-structures, in particular, induces an equivalence $\widehat{\mathrm{H}}_{c, 0^{-}} \operatorname{Mod} \stackrel{\sim}{\longrightarrow}$ $\overline{\mathrm{H}}$-Mod, of abelian categories, such that $L_{\tau}$ goes to $\tau_{\overline{\mathrm{H}}}$, for any simple $S_{n^{-}}$ module $\tau$.

For $c=\frac{1}{n}$, we expect that $\Gamma\left(\operatorname{Hilb}_{0}^{(1)}, \mathcal{V}_{c, 0} \otimes \mathrm{BKR}^{-1}\left(\right.\right.$ triv $\left.\left._{\mathrm{H}}\right)\right)$ is a 1-dimensional vector space that supports the trivial representation of the group $S_{n} \subset \mathrm{H}_{c}$.

Observe also that our conjecture would imply, in particular, that the "exotic" $t$-structure on the triangulated category $D^{b}\left(\mathrm{Coh}\left(\widehat{\operatorname{Hilb}}_{0}^{(1)}\right)\right)$ obtained by transporting 
the natural $t$-structure on $D^{b}\left(\widehat{\mathrm{H}}_{c, 0}\right.$ - Mod $)$ via the equivalence of Corollary 1.4 .3 is independent of the value of $c$; cf. subsection 7.5 .

1.6. In the special case of rank one, i.e., for $n=2$, a complete classification and explicit construction of simple $\mathrm{H}_{c}$-modules (for char $\mathbb{k}>0$ ) has been obtained by Latour [La].

It seems certain that our results in characteristic $p$ have their characteristic zero counterparts for the double-affine Hecke algebra of type $\mathbf{A}_{n-1}$, specialized at a root of unity (cf. [Ch]); in that case one has to replace Hilb ${ }^{n} \mathbb{A}^{2}$ by Hilb ${ }^{n}\left(\mathbb{C}^{*} \times \mathbb{C}^{*}\right)$; cf. $\mathrm{Ob}$.

Also, it is likely that the results of the present paper can be generalized to the case of symplectic reflection algebras associated with wreath products $\boldsymbol{\Gamma}_{n}=S_{n} \ltimes \Gamma^{n}$, where $\Gamma$ is a finite subgroup in $S L_{2}(\mathbb{k})$; see [EG, $\left.\S 11\right]$. More generally, we are going to study Azumaya algebras arising via quantum Hamiltonian reduction from the general Nakajima quiver varieties (cf. [Na2]), (of which wreath-products are special cases). Our technique is ideally suited for such a generalization, that has been, in effect, suggested earlier by Nakajima and the first author.

In another direction, the general results of $\$ 4$ below apply verbatim to quantizations of Slodowy slices considered in $\mathrm{Pr}$; see also [GG].

We are going to explore these topics elsewhere.

Acknowledgments. The authors are grateful to I. Gordon for informing one of us about his unpublished results included in [GS. Also, we would like to thank A. Premet for pointing out several inaccuracies involving restricted Lie algebras that have occurred in the original version of the paper. The research of the first and third author was partially supported by the NSF. The research of the second author was conducted for the Clay Mathematical Institute and partially supported by the CRDF award RM1-2545-MO-03 and RM1-2694, and the ANR program "GIMP", contract number ANR-05-BLAN-0029-01. He is grateful to V. Vologodsky and V. Dotsenko for patient explanations, and to the University of Chicago and Northwestern University for hospitality and support.

\section{Crystalline Differential operators.}

2.1. Unless specified otherwise, we will be working over the ground field $\mathbb{k}$, an algebraically closed field of characteristic char $\mathbb{k}=p>0$. We write $\mathrm{Fr}: \mathbb{k} \rightarrow \mathbb{k}, k \mapsto$ $k^{p}$ for the Frobenius automorphism. Given a $\mathbb{k}$-vector space $E$, it is convenient to introduce $E^{(1)}$, a vector space with the same underlying additive group as $E$, but with a "twisted" $\mathbb{k}$-linear structure given by $k \circ e:=\mathrm{Fr}^{-1}(k) \cdot e, \forall k \in \mathbb{k}, e \in E$. Note that if $A$ is a $\mathbb{k}$-algebra, then the map $A \rightarrow A, a \mapsto a^{p}$ is an additive but not $a$ $\mathbb{k}$-linear map, that becomes $\mathbb{k}$-linear if considered as a map $A^{(1)} \rightarrow A$.

Given an additive map $f: E \rightarrow F$ between two $\mathbb{k}$-vector spaces, we say that $f$ is

- $p$-linear, if $f(k \cdot e)=k^{p} \cdot f(e)$ for any $k \in k, e \in E$, i.e., if the corresponding map $E^{(1)} \rightarrow F$ is $\mathbb{k}$-linear;

- $p$-graded, if both vector spaces are equipped with $\mathbb{Z}$-gradings $E=\bigoplus E(i)$, $F=\bigoplus F(i)$, and we have $f(E(i)) \subset F(p \cdot i)$ for all $i$.

2.2. Let $X$ be a smooth algebraic variety over $\mathbb{k}$ with structure sheaf $\mathcal{O}_{X}$. Write $\mathbb{k}[X]$ for the corresponding algebra of global sections. We let $X^{(1)}$ denote an 
algebraic variety with the same structure sheaf as $X$ but with the "twisted" $\mathbb{k}$ linear structure. Thus, $\mathcal{O}_{X^{(1)}}:=\left(\mathcal{O}_{X}\right)^{(1)}$, and there is a canonical morphism Fr : $X \rightarrow X^{(1)}$ called Frobenius morphism, such that the map $f \mapsto f^{p}$ on regular functions becomes identified with the natural sheaf imbedding $\operatorname{Fr}^{\bullet} \mathcal{O}_{X^{(1)}} \hookrightarrow \mathcal{O}_{X}$.

We write $\mathcal{T}_{X}$ for the tangent sheaf on $X$, and let $T^{*} X$ denote the total space of the cotangent bundle. There is a canonical isomorphism $T^{*}\left[X^{(1)}\right] \cong\left[T^{*} X\right]^{(1)}$, and we will use the notation $T^{*} X^{(1)}$ for these two isomorphic varieties, and $\pi$ : $T^{*} X^{(1)} \rightarrow X^{(1)}$ for the natural projection. The space $T^{*} X$, resp. $T^{*} X^{(1)}$, has a canonical symplectic structure, which makes $\mathbb{k}\left[T^{*} X\right]$ a Poisson algebra.

Let $\mathcal{D}_{X}$ denote the sheaf of crystalline differential operators on $X$, that is, a sheaf of algebras generated by $\mathcal{O}_{X}$ and $\mathcal{T}_{X}$. Let $\mathcal{D}(X):=\Gamma\left(X, \mathcal{D}_{X}\right)$ denote the corresponding algebra of global sections. More generally, given a locally-free coherent sheaf (= vector bundle) $\mathcal{L}$ on $X$, let $\mathcal{D}_{X}(\mathcal{L}):=\mathcal{L} \bigotimes_{\mathcal{O}_{X}} \mathcal{D}_{X} \bigotimes_{\mathcal{O}_{X}} \mathcal{L}^{\vee}$ be the sheaf of differential operators on $\mathcal{L}$, and $\mathcal{D}(X, \mathcal{L}):=\Gamma\left(X, \mathcal{D}_{X}(\mathcal{L})\right)$ the algebra of its global sections.

2.3. The sheaf $\mathcal{D}_{X}$ is known to have a large center. Specifically, for any vector field $\xi \in \mathcal{T}_{X}$, the $p$-th power of $\xi$ acts as a derivation, hence, gives rise to another vector field, $\xi^{[p]} \in \mathcal{T}_{X}$. The assignment $\xi \mapsto \xi^{p}-\xi^{[p]}$ extends to a canonical algebra imbedding

$$
\mathbf{z}_{\mathcal{D}}: \operatorname{Sym}_{X^{(1)}} \longrightarrow \operatorname{Fr} . \mathcal{D}_{X}, \quad \xi \longmapsto \xi^{p}-\xi^{[p]}
$$

(of sheaves on $X^{(1)}$ ) whose image, to be denoted $\mathfrak{Z}_{X^{(1)}} \subset \mathrm{Fr} . \mathcal{D}_{X}$, equals the center of Fr. $\mathcal{D}_{X}$. Therefore, the isomorphism $\pi . \mathcal{O}_{T^{*} X^{(1)}} \simeq$ Sym $\mathcal{T}_{X^{(1)}}$ makes Fr. $\mathcal{D}_{X}$ a sheaf of $\pi \cdot \mathcal{O}_{T^{*} X^{(1)}}$-algebras. This way, we may (and will) view $\operatorname{Fr} . \mathcal{D}_{X}$ as a coherent sheaf on $T^{*} X^{(1)}$, to be denoted $\mathcal{D}^{(1)}$.

The sheaf $\mathcal{D}_{X}$ comes equipped with a standard increasing filtration $\mathcal{D}_{X}^{\leq k}, k=$ $0,1, \ldots$, by the order of differential operator. For the associated graded sheaf, one has a graded algebra isomorphism gr $\mathcal{D}_{X} \cong \operatorname{Sym} \mathcal{T}_{X}=\pi_{.} \mathcal{O}_{T^{*} X}$. The filtration on $\mathcal{D}_{X}$ induces a filtration on $\operatorname{Fr} . \mathcal{D}_{X}$ and also the filtration $\mathfrak{Z}_{X^{(1)}}^{\leq i}:=\operatorname{Fr} . \mathcal{D}_{X}^{\leq i} \cap$ $\mathfrak{Z}_{X^{(1)}}$ on the central subalgebra $\mathfrak{Z}_{X^{(1)}}$. Observe that the latter algebra already has a natural grading obtained, via the isomorphism $\mathfrak{Z}_{X^{(1)}}=\operatorname{Sym} \mathcal{T}_{X^{(1)}}$, from the standard grading on the Symmetric algebra. With this grading, one has a $p$-graded algebra isomorphism $\operatorname{Sym} \mathcal{T}_{X^{(1)}} \stackrel{\sim}{\longrightarrow} \operatorname{gr} \mathfrak{Z}_{X^{(1)}}$ (cf. subsection 2.1), i.e., we have $\operatorname{Sym}^{i} \mathcal{T}_{X^{(1)}} \stackrel{\sim}{\longrightarrow} \operatorname{gr}^{p \cdot i} \mathfrak{Z}_{X^{(1)}}, \forall i$.

Now, view Fr. $\mathcal{D}_{X}$ as a $\mathfrak{Z}_{X^{(1)}}$-algebra. Thus, $\operatorname{gr}\left(\operatorname{Fr} . \mathcal{D}_{X}\right)$ becomes a gr $\mathfrak{Z}_{X^{(1)}}$ algebra that may be viewed, by the isomorphism $\operatorname{Spec}\left(\operatorname{gr} \mathfrak{Z}_{X^{(1)}}\right)=T^{*} X^{(1)}$, as a $\mathbb{G}_{\mathbf{m}^{-}}$ equivariant coherent sheaf on $T^{*} X^{(1)}$. On the other hand, consider the Frobenius morphism $\mathrm{Fr}^{T^{*} X}: T^{*} X \rightarrow\left[T^{*} X\right]^{(1)}$ and view $\mathrm{Fr}^{T^{*} X} \mathcal{O}_{T^{*} X}$ as a $\mathbb{G}_{\mathbf{m}}$-equivariant coherent sheaf of algebras on $T^{*} X^{(1)}$, a $\mathbb{G}_{\mathbf{m}}$-variety. With this understood, there is a natural $\mathbb{G}_{\mathbf{m}}$-equivariant algebra isomorphism

$$
\operatorname{gr}\left(\operatorname{Fr} . \mathcal{D}_{X}\right) \simeq \pi \cdot \mathrm{Fr}^{T^{*} X} \mathcal{O}_{T^{*} X}
$$

2.4. The Rees algebra. Let $\mathrm{D}$ be an associative algebra, and write $\mathrm{D}[t]:=\mathbb{k}[t] \otimes \mathrm{D}$, where $t$ is an indeterminate. We put a grading on $\mathrm{D}[t]$ by assigning $\mathrm{D}$ grade degree zero, and setting $\operatorname{deg} t=1$. Recall that, given an increasing filtration $0=\mathrm{D}_{-1} \subset$ $\mathrm{D}_{0} \subset \mathrm{D}_{1} \subset \ldots$, on $\mathrm{D}$, such that $\mathrm{D}_{i} \cdot \mathrm{D}_{j} \subset \mathrm{D}_{i+j}$ and $\bigcup_{i \geq 0} \mathrm{D}_{i}=\mathrm{D}$, one defines the 
Rees algebra of $\mathrm{D}$ as the following graded subalgebra:

$$
\text { Rees } \mathrm{D}:=\sum_{i \geq 0} t^{i} \cdot \mathrm{D}_{i} \subset \mathrm{D}[t] .
$$

There are standard isomorphisms

$$
\left.(\mathcal{R} \text { ees } \mathrm{D})\right|_{\{0\}} \cong \operatorname{grD} \text { and }(\mathcal{R} \text { ees } \mathrm{D})_{(t)} \cong \mathbb{k}\left[t, t^{-1}\right] \otimes \mathrm{D},
$$

where, for any $\mathbb{k}[t]$-algebra $R$ we let $R_{(t)}:=\mathbb{k}\left[t, t^{-1}\right] \bigotimes_{\mathbb{k}[t]} R$ denote the localization of $R$, and for any $s \in \mathbb{k}$ we use the notation $\left.R\right|_{\{s\}}:=R /(t-s) R$.

Conversely, given a flat $\mathbb{Z}_{\geq 0}$-graded $\mathbb{k}[t]$-algebra $\mathcal{R}=\bigoplus_{i \geq 0} \mathcal{R}(i)$, set $\mathrm{D}:=$ $\left.\mathcal{R}\right|_{\{1\}}=\mathcal{R} /(t-1) \mathcal{R}$. This is a $\mathbb{k}$-algebra equipped with a canonical increasing filtration $\mathrm{D}_{i}, i=0,1, \ldots$, and with a canonical graded algebra isomorphism gr $\mathrm{D} \cong$ $\left.\mathcal{R}\right|_{\{0\}}=\mathcal{R} / t \mathcal{R}$.

The filtration on $\mathrm{D}$ is defined in the following way. Put $\mathrm{D}\left[t, t^{-1}\right]:=\mathbb{k}\left[t, t^{-1}\right] \bigotimes_{\mathbb{k}} \mathrm{D}$, and view it as a $\mathbb{Z}$-graded $\mathbb{k}\left[t, t^{-1}\right]$-algebra. Further, inverting $t$, we get from $\mathcal{R}$ a $\mathbb{Z}$-graded $\mathbb{k}\left[t, t^{-1}\right]$-algebra $\mathcal{R}_{(t)}$ that contains $\mathcal{R}$ as a $\mathbb{k}[t]$-subalgebra. The definition of $\mathrm{D}$ provides an isomorphism $\phi: \boldsymbol{\mathcal { R }}_{(t)} /(t-1) \boldsymbol{\mathcal { R }}_{(t)} \stackrel{\sim}{\longrightarrow} \mathrm{D}$ that admits a unique lift to the following graded $\mathbb{k}\left[t, t^{-1}\right]$-algebra isomorphism

$\phi_{(t)}: \mathcal{R}_{(t)} \stackrel{\sim}{\longrightarrow} \mathrm{D}\left[t, t^{-1}\right]=\mathbb{k}\left[t, t^{-1}\right] \otimes \frac{\mathcal{R}}{(t-1) \mathcal{R}}, \quad \mathcal{R}(i) \ni u \mapsto t^{i} \otimes(u \bmod (t-1) \mathcal{R})$.

The above mentioned increasing filtration on $\mathrm{D}$ is defined by

$$
\mathrm{D}_{i}:=\mathrm{D} \cap \phi_{(t)}\left(t^{-i} \cdot \mathcal{R}\right), \quad i=0,1, \ldots .
$$

Assume next that $\mathrm{D}=\bigoplus_{i \geq 0} \mathrm{D}(i)$ is a graded algebra, and view it as a filtered algebra with filtration being induced by the grading, that is, defined by $D_{i}:=$ $\bigoplus_{j \leq i} \mathrm{D}(j)$. Then, we have

$$
\text { Rees } \mathrm{D}=\sum_{i \geq 0} t^{i} \cdot\left(\bigoplus_{j \leq i} \mathrm{D}(j)\right)=\left(\sum_{i \geq 0} t^{i} \cdot \mathrm{D}(i)\right)[t] .
$$

We see that, for a graded algebra $\mathrm{D}$, one has the following graded algebra isomorphism:

$$
\mathbb{k}[t] \otimes \mathrm{D} \stackrel{\sim}{\longrightarrow} \text { Rees } \mathrm{D}, \quad \mathbb{k}[t] \bigotimes\left(\oplus_{i} \mathrm{D}(i)\right) \ni f \otimes\left(\sum_{i} u_{i}\right) \longmapsto f \cdot \sum_{i} t^{i} \cdot u_{i} .
$$

2.5. The sheaf $\mathcal{R} \mathcal{D}^{(1)}$. We apply the Rees algebra construction to $\mathrm{Fr} . \mathcal{D}_{X}$, viewed as a sheaf of filtered algebras. Thus, we get a sheaf $\mathcal{R} e e s$ Fr. $\mathcal{D}_{X}$ of graded $\mathcal{O}_{X^{(1)}}$-algebras. In $\mathcal{R}$ ees $\operatorname{Fr} . \mathcal{D}_{X}$, we also have a central subalgebra $\mathcal{R}$ ees $\mathfrak{Z}_{X^{(1)}} \subset$ Rees $\operatorname{Fr} . \mathcal{D}_{X}$.

By (2.4.3), the canonical grading on $\mathfrak{Z}_{X^{(1)}}$ provides a $p$-graded algebra isomorphism $\mathcal{R}$ ees $\mathfrak{Z}_{X^{(1)}} \simeq \mathbb{k}[t] \otimes \mathfrak{Z}_{X^{(1)}}$. Thus, we obtain the following canonical algebra maps

$\operatorname{Sym} \mathcal{T}_{X^{(1)}} \stackrel{(2.3 .1)}{\sim} \mathfrak{Z}_{X^{(1)}} \stackrel{z \mapsto 1 \otimes z}{\longrightarrow} \mathbb{k}[t] \otimes \mathfrak{Z}_{X^{(1)}} \stackrel{(2.4 .3)}{=} \mathcal{R}$ ees $\mathfrak{Z}_{X^{(1)}} \longrightarrow$ Rees $\mathrm{Fr} . \mathcal{D}_{X}$.

The composite map in (2.5.1) is a p-graded map to be denoted $\mathbf{z}_{\mathcal{R}_{\mathcal{D}}}$. This map specializes at $t=0$ to the map $\xi \mapsto \xi^{p}$, and at $t=1$ to (2.3.1).

Furthermore, the algebra isomorphism $\mathcal{R}$ ees $\mathfrak{Z}_{X^{(1)}} \simeq \mathbb{k}[t] \otimes \mathfrak{Z}_{X^{(1)}}$ yields a direct product decomposition

$$
\operatorname{Spec}\left(\mathcal{R} \text { ees } \mathfrak{Z}_{X^{(1)}}\right) \cong \mathbb{A}^{1} \times \operatorname{Spec} \mathfrak{Z}_{X^{(1)}}=\mathbb{A}^{1} \times T^{*} X^{(1)} .
$$


We will often identify $\mathcal{R}$ ees $\operatorname{Fr} . \mathcal{D}_{X}$, a graded $\mathcal{R}$ ees $\mathfrak{Z}_{X^{(1)}}$-algebra, with a coherent sheaf of algebras on $\operatorname{Spec}\left(\mathcal{R}\right.$ ees $\left.\mathfrak{Z}_{X^{(1)}}\right)$, that is, on $\mathbb{A}^{1} \times T^{*} X^{(1)}$. The resulting sheaf on $\mathbb{A}^{1} \times T^{*} X^{(1)}$, to be denoted $\mathcal{R} \mathcal{D}^{(1)}$, is easily seen to be flat with respect to the first factor $\mathbb{A}^{1}$. Moreover, (2.4.1) yields, in view of (2.3.2), the following isomorphisms of sheaves of algebras on $T^{*} X^{(1)}$, resp., on $\left(\mathbb{A}^{1} \backslash\{0\}\right) \times T^{*} X^{(1)}$ :

$$
\left.\left(\mathcal{R} \mathcal{D}^{(1)}\right)\right|_{\{0\} \times T^{*} X^{(1)}} \cong \operatorname{Fr}^{T^{*} X} \mathcal{O}_{T^{*} X},\left.\quad\left(\mathcal{R} \mathcal{D}^{(1)}\right)\right|_{\left(\mathbb{A}^{1} \backslash\{0\}\right) \times T^{*} X^{(1)}} \cong \operatorname{pr}_{2}^{*}\left(\mathcal{D}^{(1)}\right),
$$

where $\operatorname{pr}_{2}:\left(\mathbb{A}^{1} \backslash\{0\}\right) \times T^{*} X^{(1)} \rightarrow T^{*} X^{(1)}$ is the second projection.

Moreover, the grading on the Rees algebra $\mathcal{R}$ ees $\mathfrak{Z}_{X^{(1)}}$ makes $\operatorname{Spec}\left(\mathcal{R}\right.$ ees $\left.\mathfrak{Z}_{X^{(1)}}\right)$ a $\mathbb{G}_{\mathbf{m}}$-variety. It follows from formula (2.4.3) that the natural $\mathbb{G}_{\mathbf{m}}$-action on $\operatorname{Spec}\left(\mathcal{R}\right.$ ees $\left.\mathfrak{Z}_{X^{(1)}}\right)$ corresponds, via (2.5.2), to the $\mathbb{G}_{\mathbf{m}^{-}}$diagonal action on $\mathbb{A}^{1} \times$ $T^{*} X^{(1)}$. The sheaf $\mathcal{R} \mathcal{D}^{(1)}$ on $\mathbb{A}^{1} \times T^{*} X^{(1)}$ comes equipped with a canonical $\mathbb{G}_{\mathbf{m}^{-}}$ equivariant structure.

\section{HAMILTONIAN REDUCTION IN CHARACTERISTIC $p$}

3.1. Lie algebras in characteristic $p$. Let $A$ be a connected linear algebraic group over $\mathbb{k}$. Write $A^{(1)}$ for the Frobenius twist of $A$ (cf. \$2), an algebraic group isomorphic to $A$ and equipped with an algebraic group morphism $\mathrm{Fr}: A \rightarrow A^{(1)}$, called the Frobenius morphism. The kernel of this morphism is an infinitesimal group scheme $A_{1} \subset A$, called Frobenius kernel. By definition, one has an exact sequence:

$$
1 \longrightarrow A_{1} \longrightarrow A \stackrel{\mathrm{Fr}}{\longrightarrow} A^{(1)} \longrightarrow 1 .
$$

The Lie algebra $\mathfrak{a}:=$ Lie $A$ may be viewed as the vector space of left invariant vector fields on $A$. This vector space comes equipped with a natural structure of $p$-Lie algebra, i.e., we have a $p$-power map $\mathfrak{a} \rightarrow \mathfrak{a}, x \mapsto x^{[p]}$; see [J] or [Ja].

Let $S y m \mathfrak{a}$, resp. $\mathcal{U} \mathfrak{a}$, be the symmetric, resp. enveloping, algebra of $\mathfrak{a}$. The group $A$ acts on Sym $\mathfrak{a}$ and $\mathcal{U} \mathfrak{a}$ by algebra automorphisms via the adjoint action.

The standard increasing filtration $\mathcal{U}_{\mathbf{0}} \mathfrak{a}$ on the enveloping algebra gives rise to a graded algebra $\mathcal{R}$ ees $\mathcal{U} \mathfrak{a}=\sum_{i \geq 0} t^{i} \cdot \mathcal{U}_{i} \mathfrak{a}$. Jacobson's argument [J], ch. V,, 7 , (60)-(64)] shows that the following assignment

$$
\mathbf{z}_{\mathcal{R U}}: \quad \operatorname{Syma} \mathfrak{a}^{(1)} \longrightarrow \mathcal{R} \text { ees } \mathcal{U} \mathfrak{a}, \quad \mathfrak{a}^{(1)} \ni x \longmapsto x^{p}-t^{p-1} \cdot x^{[p]}
$$

gives a well-defined Ad $A$-equivariant injective $p$-graded algebra homomorphism; cf. also [PS]. The image of the map $\mathbf{z}_{\mathcal{R} \mathcal{U}}$ is an $\mathrm{Ad} A$-stable subalgebra contained in the center of $\mathcal{R e e s} \mathcal{U}$ a.

Specialization of the map $\mathbf{z}_{\mathcal{R} \mathcal{U}}$ at $t=0$ reduces to the p-graded algebra map Sym $\mathfrak{a}^{(1)} \hookrightarrow \operatorname{Sym} \mathfrak{a}, x \mapsto x^{p}$. On the other hand, specializing the map $\mathbf{z}_{\mathcal{R} u}$ at $t=1$, one obtains an algebra imbedding $\mathbf{z}_{\mathcal{U}}:=\left.\mathbf{z}_{\mathcal{R} \mathcal{U}}\right|_{t=1}:$ Sym $\mathfrak{a}^{(1)} \hookrightarrow \mathcal{U} \mathfrak{a}$. The image $\mathfrak{Z}(\mathfrak{a}):=\mathbf{z}_{\mathcal{U}}\left(\right.$ Sym $\left.\mathfrak{a}^{(1)}\right)$ of this imbedding is a central subalgebra in $\mathcal{U} \mathfrak{a}$ generated by the elements $\left\{x^{p}-x^{[p]}\right\}_{x \in \mathfrak{a}}$, usually referred to as the $p$-center of $\mathcal{U} \mathfrak{a}$; cf. e.g. Ja]. This way, the map in (3.1.1) may be identified with the composite of the following chain of algebra homomorphisms, completely analogous to those in (2.5.1):

$$
\operatorname{Sym} \mathfrak{a}^{(1)} \stackrel{\mathbf{z}_{\mathcal{U}}}{\longrightarrow} \mathfrak{Z}(\mathfrak{a}) \stackrel{z \mapsto 1 \otimes z}{\longrightarrow} \mathbb{k}[t] \otimes \mathfrak{Z}(\mathfrak{a}) \stackrel{(2.4 .3)}{=} \mathcal{R e e s} \mathfrak{Z}(\mathfrak{a}) \longleftrightarrow \text { Rees } \mathcal{U} \mathfrak{a} .
$$

The adjoint action on $\mathfrak{Z}(\mathfrak{a})$ of the Frobenius kernel $A_{1} \subset A$ is trivial, hence, the $A$-action on $\mathfrak{Z}(\mathfrak{a})$ factors through $A^{(1)}$. 
3.2. The Artin-Schreier map. Let $\mathfrak{a}^{*}$ denote the $\mathbb{k}$-linear dual of $\mathfrak{a}$, and write $\mathfrak{a}^{1, *}:=\left(\mathfrak{a}^{(1)}\right)^{*}$ for the $\mathbb{k}$-linear dual of $\mathfrak{a}^{(1)}$. For any linear function $\lambda \in \mathfrak{a}^{*}$, the assignment $x \mapsto \lambda(x)^{p}$ gives a $p$-linear map $\mathfrak{a} \rightarrow \mathbb{k}$, that is, $\mathbb{k}$-linear function on $\mathfrak{a}^{(1)}$, to be denoted $\lambda^{(1)}$. This way, one obtains a $p$-linear map $\mathfrak{a}^{*} \rightarrow\left(\mathfrak{a}^{(1)}\right)^{*}, \lambda \mapsto \lambda^{(1)}$. The latter map gives a canonical $\mathbb{k}$-vector space isomorphism $\left(\mathfrak{a}^{*}\right)^{(1)} \stackrel{\sim}{\longrightarrow}\left(\mathfrak{a}^{(1)}\right)^{*}, \lambda \mapsto$ $\lambda^{(1)}$.

Let $\mathbb{X}^{*}(\mathfrak{a}) \subset \mathfrak{a}^{*}$ denote the subspace of fixed points of the coadjoint action of $A$ on $\mathfrak{a}^{*}$. Such a fixed point may be viewed as an $\operatorname{Ad} A$-invariant Lie algebra homomorphism $\mathfrak{a} \rightarrow \mathbb{k}$ (note that a Lie algebra homomorphism $\mathfrak{a} \rightarrow \mathbb{k}$ need not necessarily be $\operatorname{Ad} A$-invariant).

Given $\chi \in \mathbb{X}^{*}(\mathfrak{a})$, we write $\chi^{[1]}$ for the function $\mathfrak{a} \rightarrow \mathbb{k}, x \mapsto \chi\left(x^{[p]}\right)$.

Lemma 3.2.1. For any $\chi \in \mathbb{X}^{*}(\mathfrak{a})$, the function $\chi^{[1]}: \mathfrak{a} \rightarrow \mathbb{k}$ is a p-linear map, that is, $\chi^{[1]} \in \mathfrak{a}^{1, *}$.

Proof. The above mentioned Jacobson's formula implies that, in $\mathcal{U} \mathfrak{a}$, one has an equality (cf. also [Ja, Lemma 2.1]):

$$
(x+y)^{[p]}-x^{[p]}-y^{[p]}=(x+y)^{p}-x^{p}-y^{p} .
$$

Now, extend $\chi$ to an algebra homomorphism $\mathcal{U} \mathfrak{a} \rightarrow \mathbb{k}$, and apply the resulting map to the equation above. We find

$$
\begin{aligned}
\chi\left((x+y)^{[p]}-x^{[p]}-y^{[p]}\right) & =\chi\left((x+y)^{p}-x^{p}-y^{p}\right)=\chi(x+y)^{p}-\chi(x)^{p}-\chi(y)^{p} \\
& =(\chi(x)+\chi(y))^{p}-\chi(x)^{p}-\chi(y)^{p}=0 .
\end{aligned}
$$

The lemma follows.

Lemma 3.2.1 shows that the assignment $\chi \mapsto \chi^{[1]}$, as well as the assignment

$$
\varkappa: \mathbb{X}^{*}(\mathfrak{a}) \longrightarrow \mathfrak{a}^{1, *}, \quad \varkappa(\chi)=\chi^{(1)}-\chi^{[1]}: x \longmapsto \chi(x)^{p}-\chi\left(x^{[p]}\right),
$$

gives a well-defined $p$-linear map $\mathbb{X}^{*}(\mathfrak{a}) \longrightarrow \mathfrak{a}^{1, *}$. The map (3.2.2) will play an important role later in this paper, it may be thought of as a Lie algebra analogue of the Artin-Schreier map.

Let $\mathcal{I}_{\varphi} \subset \operatorname{Syman}^{(1)}=\mathbb{k}\left[\mathfrak{a}^{1, *}\right]$ denote the maximal ideal corresponding to a point $\varphi \in \mathfrak{a}^{1, *}$.

Now, given $\chi \in \mathbb{X}^{*}(\mathfrak{a})$, extend it to an algebra map $\chi: \mathcal{U} \mathfrak{a} \rightarrow \mathbb{k}$, as in the proof of Lemma 3.2.1.

Corollary 3.2.3. Let $\chi \in \mathbb{X}^{*}(\mathfrak{a})$. For the composite homomorphism below we have

$$
\operatorname{Ker}\left[\operatorname{Sym}_{\mathfrak{a}}^{(1)} \underset{(3.1 .1)}{\mathbf{z}_{\mathcal{U}}} \mathcal{U} \mathfrak{a} \stackrel{\chi}{\longrightarrow} \mathbb{k}\right]=\mathcal{I}_{\varkappa(\chi)} .
$$

Proof. For $x \in \mathfrak{a}$, we compute $\chi\left(\mathbf{z}_{\mathcal{U}}(x)\right)=\chi\left(x^{p}-x^{[p]}\right)=\chi(x)^{p}-\chi\left(x^{[p]}\right)=\varkappa(\chi)(x)$.

Let $\mathbb{X}^{*}(A):=\operatorname{Hom}\left(A, \mathbb{G}_{\mathbf{m}}\right)$ be the character lattice of the algebraic group $A$. The differential of a character $f: A \rightarrow \mathbb{G}_{\mathbf{m}}$ at $1 \in A$ is a linear function $x \mapsto x(f)(1)$ on the Lie algebra $\mathfrak{a}$, which is clearly an element of $\mathbb{X}^{*}(\mathfrak{a})$. We denote this linear function by $\operatorname{dlog} f$, so that the assignment $f \mapsto \operatorname{dlog} f$ yields an additive group homomorphism dlog: $\mathbb{X}^{*}(A) \rightarrow \mathbb{X}^{*}(\mathfrak{a})$.

Fix $f \in \mathbb{X}^{*}(A)$ and put $\phi:=\operatorname{dlog} f \in \mathbb{X}^{*}(\mathfrak{a})$. Observe that, for any $x \in \mathfrak{a}$ viewed as a left invariant vector field on $A$, we have $x(f)=\phi(x) \cdot f$. It follows that the 
$p$-th power of $x$, viewed as a left invariant differential operator on $A$ of order $p$, acts on a character $f \in \mathbb{X}^{*}(A)$ as multiplication by the constant $\phi(x)^{p} \in \mathbb{k}$. On the other hand, this differential operator is a derivation, which corresponds to the left invariant vector field $x^{[p]}$. Thus, we also have $x^{[p]}(f)=\phi\left(x^{[p]}\right) \cdot f$. Combining together the equations above, we deduce $\phi\left(x^{[p]}\right)=\phi(x)^{p}, \forall x \in \mathfrak{a}$, that is, $\varkappa(\phi)=0$. Thus, we have proved

$$
\operatorname{d} \log \left(\mathbb{X}^{*}(A)\right) \subset \operatorname{Ker}\left[\varkappa: \mathbb{X}^{*}(\mathfrak{a}) \longrightarrow \mathfrak{a}^{1, *}\right]
$$

3.3. Restricted enveloping algebras. Fix $\chi \in \mathbb{X}^{*}(\mathfrak{a})$, and let $\chi: \mathcal{U} \mathfrak{a} \rightarrow \mathbb{k}$ be the corresponding algebra homomorphism.

Definition 3.3.1. Let $I_{\chi}:=\operatorname{Ker}(\mathcal{U} \mathfrak{a} \rightarrow \mathbb{k})$ denote the kernel of $\chi$, the two-sided ideal in $\mathcal{U} \mathfrak{a}$ generated by the elements $\{x-\chi(x)\}_{x \in \mathfrak{a}}$. Also, in $\mathfrak{Z}(\mathfrak{a})$, consider the following ideal:

$$
I_{\chi}^{(1)}:=I_{\chi} \cap \mathfrak{Z}(\mathfrak{a}) \subset \mathfrak{Z}(\mathfrak{a}) \subset \mathcal{U} \mathfrak{a}, \quad \text { and set } \mathfrak{u}_{\chi}(\mathfrak{a}):=\mathcal{U} \mathfrak{a} / \mathcal{U} \mathfrak{a} \cdot I_{\chi}^{(1)} .
$$

Here, $\mathcal{U} \mathfrak{a} \cdot I_{\chi}^{(1)} \subset I_{\chi}$, is an Ad $A$-stable two-sided ideal in $\mathcal{U} \mathfrak{a}$, and the quotient $\mathfrak{u}_{\chi}(\mathfrak{a})$ is an associative algebra of dimension $\operatorname{dim} \mathfrak{u}_{\chi}(\mathfrak{a})=p^{\operatorname{dim} \mathfrak{a}}$, called $\chi$-restricted enveloping algebra. By definition there is an exact sequence

$$
0 \longrightarrow \mathcal{U} \mathfrak{a} \cdot I_{\chi}^{(1)} \longrightarrow \mathcal{U} \mathfrak{a} \stackrel{r}{\longrightarrow} \mathfrak{u}_{\chi}(\mathfrak{a}) \longrightarrow 0
$$

Corollary 3.2 .3 shows that $I_{\chi}^{(1)}$ is a maximal ideal in $\mathfrak{Z}(\mathfrak{a})$ that goes, under the isomorphisms $\mathfrak{Z}(\mathfrak{a}) \cong \operatorname{Sym} \mathfrak{a}^{(1)} \cong \mathbb{k}\left[\mathfrak{a}^{1, *}\right]$, to the maximal ideal in $\mathbb{k}\left[\mathfrak{a}^{1, *}\right]$ corresponding to the point $\varkappa(\chi) \in \mathfrak{a}^{1, *}$, that is, to the ideal $\mathcal{I}_{\varkappa(\chi)}$. Thus, we have

$$
I_{\chi}^{(1)}=\mathbf{z}_{\mathcal{U}}\left(\mathcal{I}_{\varkappa(\chi)}\right), \quad \text { hence } \quad \mathfrak{u}_{\chi}(\mathfrak{a})=\mathcal{U} \mathfrak{a} / \mathcal{U} \mathfrak{a} \cdot \mathbf{z}_{\mathcal{U}}\left(\mathcal{I}_{\varkappa(\chi)}\right) \text {. }
$$

Observe further that, the $A_{1}$-action on $\mathfrak{Z}(\mathfrak{a})$ being trivial, it preserves the ideal $\mathcal{U} \mathfrak{a} \cdot I_{\chi}^{(1)}$, hence induces a well-defined $A_{1}$-action on $\mathfrak{u}_{\chi}(\mathfrak{a})$ by algebra automorphisms. We set

$$
\mathfrak{i}_{\chi}:=r\left(I_{\chi}\right)=I_{\chi} / \mathcal{U} \mathfrak{a} \cdot I_{\chi}^{(1)} \subset \mathfrak{u}_{\chi}(\mathfrak{a})
$$

Thus, $\mathfrak{i}_{\chi}$ is an $A_{1}$-stable two-sided ideal in $\mathfrak{u}_{\chi}(\mathfrak{a})$ generated by the elements $\{x-\chi(x)\}_{x \in \mathfrak{a}}$.

In the special case $\chi=0$, the restricted enveloping algebra $\mathfrak{u}_{0}(\mathfrak{a}):=\mathcal{U} \mathfrak{a} / \mathcal{U} \mathfrak{a} \cdot I_{0}^{(1)}$ inherits from $\mathcal{U} \mathfrak{a}$ the structure of a Hopf algebra. This Hopf algebra is dual to $\mathbb{k}\left[A_{1}\right]$, the coordinate ring of the Frobenius kernel $A_{1}$.

For any $\chi$, the map ad $x: u \mapsto x \cdot u-u \cdot x, x \in \mathfrak{a}, u \in \mathfrak{u}_{\chi}(\mathfrak{a})$, extends to a well-defined $\mathfrak{u}_{0}(\mathfrak{a})$-action on $\mathfrak{u}_{\chi}(\mathfrak{a})$, that is, to an algebra map ad $: \mathfrak{u}_{0}(\mathfrak{a}) \longrightarrow$ $\operatorname{End}_{\mathbb{k}}\left(\mathfrak{u}_{\chi}(\mathfrak{a})\right)$. This $\mathfrak{u}_{0}(\mathfrak{a})$-action corresponds to the adjoint action on $\mathfrak{u}_{\chi}(\mathfrak{a})$ of the Frobenius kernel $A_{1}$.

3.4. Quantum Hamiltonian reduction. Let D be any associative, not necessarily commutative, $\mathbb{k}$-algebra equipped with an algebraic action of the group $A$ by algebra automorphisms and with an $A$-equivariant algebra map $\rho: \mathcal{U} \mathfrak{a} \rightarrow \mathrm{D}$ such that the adjoint $\mathfrak{a}$-action on $\mathrm{D}$, given by ad $x: u \mapsto \rho(x) \cdot u-u \cdot \rho(x), x \in \mathfrak{a}, u \in \mathrm{D}$, is equal to the differential of the $A$-action.

Let $I \subset \mathcal{U} \mathfrak{a}$ be an Ad $A$-stable two-sided ideal. Then, D. $\rho(I)$ is an $A$-stable left ideal in $\mathrm{D}$. It is easy to verify that multiplication in $\mathrm{D}$ descends to a well-defined associative algebra structure on $(\mathrm{D} / \mathrm{D} \cdot \rho(I))^{A}$, the space of $A$-invariants in $\mathrm{D} / \mathrm{D} \cdot \rho(I)$.

Abusing notation, from now on we will write $\mathrm{D} \cdot I$ instead of $\mathrm{D} \cdot \rho(I)$. 
Remark 3.4.1. The algebra (D/D $I)^{A}$ may be thought of as a "Hamiltonian reduction" of D with respect to $I$.

Observe also that, if $u \in \mathrm{D}$ is such that $u \bmod (\mathrm{D} \cdot I) \in(\mathrm{D} / \mathrm{D} \cdot I)^{A}$, then the operator of right multiplication by $u$ descends to a well-defined map $R_{u}: \mathrm{D} / \mathrm{D} \cdot I$ $\rightarrow \mathrm{D} / \mathrm{D} \cdot I$. Moreover, the assignment $u \mapsto R_{u}$ induces an algebra isomorphism $(\mathrm{D} / \mathrm{D} \cdot I)^{A} \stackrel{\sim}{\longrightarrow}\left(\operatorname{End}_{\mathrm{D}}(\mathrm{D} / \mathrm{D} \cdot I)\right)^{\mathrm{opp}}$.

More generally, let $M$ be a right $\mathrm{D}$-module equipped with an $A$-equivariant structure (i.e., such that the action map $M \otimes \mathrm{D} \rightarrow M$ is $A$-equivariant). The algebra map $\mathcal{U} \mathfrak{a} \rightarrow \mathrm{D}$ makes $M$ an $\mathcal{U} \mathfrak{a}$-module. The space $(M / M \cdot I)^{A}$ acquires a natural right $(\mathrm{D} / \mathrm{D} \cdot I)^{A}$-module structure, to be called a Hamiltonian reduction of $M$.

Next, fix $\chi \in \mathbb{X}^{*}(\mathfrak{a})$, and let $\mathfrak{u}_{\chi}(\mathfrak{a})$ be the corresponding $\chi$-restricted enveloping algebra. Recall that this algebra comes equipped with the adjoint action of $A_{1}$, the Frobenius kernel. Let $\mathrm{D}$ be an associative algebra equipped with $A_{1}$-action, and with $\rho: \mathfrak{u}_{\chi}(\mathfrak{a}) \rightarrow \mathrm{D}$, an $A_{1}$-equivariant algebra morphism. One shows similarly that, given an $A_{1}$-stable two-sided ideal $\mathfrak{i} \subset \mathfrak{u}_{\chi}(\mathfrak{a})$, there is a natural associative algebra structure on $(\mathrm{D} / \mathrm{D} \cdot \mathfrak{i})^{A_{1}}$. In the special case $\mathfrak{i}=\mathfrak{i}_{\chi}$ (see (3.3.4) ), the algebra $\left(\mathrm{D} / \mathrm{D} \cdot \mathfrak{i}_{\chi}\right)^{A_{1}}$ may be thought of as a quantum Hamiltonian reduction of $\mathrm{D}$ with respect to the action of $A_{1}$, an "infinitesimal" group-scheme.

Later on, we will be interested in the following special case of this construction. Let $E$ be a finite dimensional $A$-module such that the induced action map $\rho: \mathcal{U} \mathfrak{a} \rightarrow$ End $E$ descends to the algebra $\mathfrak{u}_{\chi}(\mathfrak{a})$, i.e., vanishes on the ideal $I_{\chi}^{(1)} \subset \mathfrak{Z}(\mathfrak{a})$. Write $E_{\chi}:=\{e \in E \mid x(e)=\chi(x) \cdot e, \forall x \in \mathfrak{a}\}$ for the $\chi$-weight space of $\mathcal{U} \mathfrak{a}$.

We put $\mathrm{D}:=$ End $E\left(=\operatorname{End}_{\mathbb{k}} E\right)$. We claim that the quantum Hamiltonian reduction of the algebra $D$ with respect to the $\mathfrak{u}_{\chi}(\mathfrak{a})$-action is canonically isomorphic to $\operatorname{End}_{\mathbb{k}} E_{\chi}$.

In more detail, we form an associative algebra $D_{\chi}:=\left(D / D \cdot i_{\chi}\right)^{A_{1}}$. The natural action of $\mathrm{D}$ on $E$ descends to a well-defined $\mathrm{D}_{\chi}$-action on the weight space $E_{\chi}$. On the other hand, we have a right $D_{\chi}$-action on $D / D \cdot i_{\chi}$.

We are going to establish canonical algebra isomorphisms

$$
\operatorname{End}_{\mathrm{D}}\left(\mathrm{D} / \mathrm{D} \cdot \mathrm{i}_{\chi}\right)^{\text {opp }} \stackrel{\mathrm{l}}{\cong} \mathrm{D}_{\chi} \stackrel{\mathrm{r}}{\cong} \operatorname{End}_{\mathrm{k}} E_{\chi} \text {. }
$$

The isomorphisms above follow from a more general result below that involves two characters $\chi, \psi \in \mathbb{X}^{*}(\mathfrak{a})$, such that $\varkappa(\chi)=\varkappa(\psi)$. In this case, in $\mathcal{U} \mathfrak{a}$ we have $I_{\chi}^{(1)}=I_{\psi}^{(1)}$. Hence there is a canonical identification $\mathfrak{u}_{\chi}(\mathfrak{a})=\mathfrak{u}_{\psi}(\mathfrak{a})$, and we may view the $A$-representation $E$ either as an $\mathfrak{u}_{\chi}(\mathfrak{a})$ - or as an $\mathfrak{u}_{\psi}(\mathfrak{a})$-module. We set $\mathrm{D}:=\operatorname{End}_{\mathrm{k}} E$, as above, and consider the Hamiltonian reductions $\mathrm{D}_{\chi}$ and $\mathrm{D}_{\psi}$. We have a $\mathrm{D}_{\chi}$-action on the weight space $E_{\chi} \subset E$, and a similar $\mathrm{D}_{\psi}$-action on the weight space $E_{\psi}$.

Observe that the natural D-bimodule structure on the algebra D, via left and right multiplication, descends to a $\mathrm{D}-\mathrm{D}_{\chi}$-bimodule structure on the vector space $\operatorname{Hom}_{\mathbb{k}}\left(E_{\chi}, E\right)$. Furthermore, the right $\mathrm{D}_{\chi}$-action on $\mathrm{D} / \mathrm{D} \cdot \mathfrak{i}_{\chi}$, resp., $\mathrm{D}_{\psi}$-action on $\mathrm{D} / \mathrm{D} \cdot \mathfrak{i}_{\psi}$, gives the following space a natural $\mathrm{D}_{\chi}-\mathrm{D}_{\psi}$-bimodule structure:

$$
{ }_{\psi} \mathrm{D}_{\chi}:=\operatorname{Hom}_{\mathrm{D}}\left(\mathrm{D} / \mathrm{D} \cdot \mathfrak{i}_{\chi}, \mathrm{D} / \mathrm{D} \cdot \mathfrak{i}_{\psi}\right)^{\psi-\chi},
$$

where the superscript " $\psi-\chi$ " denotes the $(\psi-\chi)$-weight component of the natural (adjoint) $A$-action. 
Lemma 3.4.4. (i) The restriction map End $E \rightarrow \operatorname{Hom}\left(E_{\chi}, E\right)$, resp. End $E \rightarrow$ $\operatorname{Hom}\left(E_{\psi}, E\right)$, induces a $\mathrm{D}_{-} \mathrm{D}_{\chi}$-bimodule, resp. $\mathrm{D}_{-} \mathrm{D}_{\psi}$-bimodule, isomorphism

$$
\mathrm{D} / \mathrm{D} \cdot \mathfrak{i}_{\chi} \stackrel{\text { res }}{\longrightarrow} \operatorname{Hom}_{\mathbb{k}}\left(E_{\chi}, E\right), \quad \text { resp., } \quad \mathrm{D} / \mathrm{D} \cdot \mathfrak{i}_{\psi} \stackrel{\stackrel{\text { res }}{\longrightarrow}}{\longrightarrow} \operatorname{Hom}_{\mathbb{k}}\left(E_{\psi}, E\right) .
$$

(ii) We have the following $\mathrm{D}_{\chi}-\mathrm{D}_{\psi}$-bimodule isomorphisms:

$$
{ }_{\psi} \mathrm{D}_{\chi} \stackrel{\text { res }}{\longrightarrow} \operatorname{Hom}_{\mathrm{D}}\left(\operatorname{Hom}_{\mathbb{k}}\left(E_{\chi}, E\right), \operatorname{Hom}_{\mathbb{k}}\left(E_{\psi}, E\right)\right)^{\psi-\chi} \stackrel{\sim}{\sim} \operatorname{Hom}_{\mathbb{k}}\left(E_{\chi}, E_{\psi}\right) .
$$

For $\chi=\psi$, it follows that the map $\mathbf{l}$ in (3.4.2) is an algebra isomorphism, furthermore, the composite map in (3.4.5) induces an algebra isomorphism $\mathrm{D}_{\chi}=$ ${ }_{\chi} \mathrm{D}_{\chi} \stackrel{\sim}{\longrightarrow} \operatorname{End}_{\mathbb{k}}\left(E_{\chi}\right)$, which gives the isomorphism $\mathbf{r}$ in (3.4.2).

Sketch of Proof. Associated to a vector subspace $F \subset E$, one has a left ideal $J_{F} \subset$ $\mathrm{D}$, defined by $J_{F}:=\left\{f \in \mathrm{D}=\operatorname{End}_{\mathbb{k}} E|f|_{F}=0\right\}$. Moreover, any left ideal $J \subset \mathrm{D}$ has the form $J=J_{F}$ where the corresponding subspace $F \subset E$ can be recovered from $J$ by the formula $F=\bigcap_{f \in J} \operatorname{Ker} f$. Applying this to the left ideal $J=\mathrm{D} \cdot \mathfrak{i}_{\chi}$ we get $\mathbf{D} \cdot \mathfrak{i}_{\chi}=\left\{f \in \operatorname{End}_{\mathbb{k}} E|f|_{F}=0\right\}$, where $F=\bigcap_{x \in \mathfrak{i}_{\chi}} \operatorname{Ker} \rho(x)$. The latter space equals $E_{\chi}$, by definition. Thus, we deduce $\mathrm{D} \cdot \mathfrak{i}_{\chi}=\left\{f \in \operatorname{End}_{\mathbb{k}} E|f|_{E_{\chi}}=0\right\}$ $=\operatorname{Hom}_{\mathbb{k}}\left(E / E_{\chi}, E\right)$, hence $\mathrm{D} / \mathrm{D} \cdot \mathfrak{i}_{\chi} \cong \operatorname{Hom}_{\mathbb{k}}\left(E_{\chi}, E\right)$.

The rest of the proof is an elementary exercise which we leave for the reader.

3.5. Moment maps. Let $A$ be a linear algebraic group as in subsection 3.1, and let $A \times X \rightarrow X$ be an algebraic action on $X$, a smooth $\mathbb{k}$-variety. Any element $x \in \mathfrak{a}$ gives rise to an algebraic vector field $\xi_{x}$ on $X$. We may view $\xi_{x}$ as a regular function on $T^{*} X$. This way, the assignment $x \mapsto \xi_{x}$ extends uniquely to an $A$-equivariant Poisson algebra map $\mu_{\text {alg }}: \operatorname{Sym} \mathfrak{a} \rightarrow \mathbb{k}\left[T^{*} X\right]$. Since Sym $\mathfrak{a} \cong \mathbb{k}\left[\mathfrak{a}^{*}\right]$, this algebra map induces an $A$-equivariant morphism $\mu: T^{*} X \rightarrow \mathfrak{a}^{*}$, called moment map, such that the algebra map $\mu_{\text {alg }}$ becomes the pull-back via $\mu$.

There is also a noncommutative analogue of the Poisson algebra map $\mu_{\text {alg }}$. Specifically, the Lie algebra morphism $x \mapsto \xi_{x}$ extends uniquely to an $A$-equivariant associative algebra homomorphism $\mu_{\mathfrak{U}}: \mathcal{U} \mathfrak{a} \rightarrow \mathcal{D}_{X}$ (more generally, given an $A$ equivariant vector bundle $\mathcal{L}$, one defines similarly an associative algebra homomorphism $\mu_{\mathcal{U}}: \mathcal{U} \mathfrak{a} \rightarrow \mathcal{D}(X, \mathcal{L})$ ). The morphism $\mu_{\mathcal{U}}$ is compatible with natural filtrations, hence, induces a canonical graded algebra homomorphism $\mu_{\mathcal{R}}: \mathcal{R e e s} \mathcal{U} \mathfrak{a} \longrightarrow$ $\boldsymbol{R}$ ees $\mathcal{D}_{X}$. We may view the later homomorphism as a map $\mathcal{R}$ ees $\mathcal{U} \mathfrak{a} \longrightarrow \pi \cdot \mathcal{R}^{(1)}$; cf. subsection 2.5.

We have the following commutative diagram:

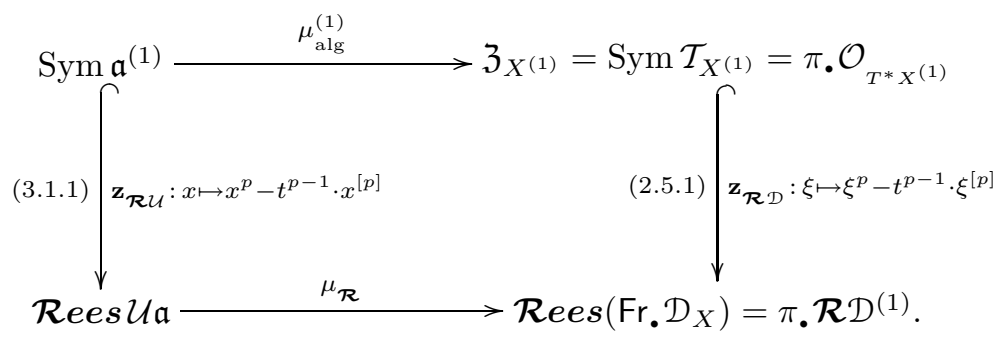

The map $\mu_{\mathcal{R}}$ in the bottom row specializes at $t=1$ to the map $\left.\mu_{\mathcal{R}}\right|_{t=1}=\mu_{\mathcal{U}}$ : $\mathcal{U} \mathfrak{a} \rightarrow \mathcal{D}_{X}$ considered earlier, and specializes at $t=0$ to the map $\left.\mu_{\mathcal{R}}\right|_{t=0}=\mu_{\text {alg }}$. The vertical maps in diagram (3.5.1) are the $p$-graded algebra morphisms considered earlier. 
Furthermore, fix $\chi \in \mathbb{X}^{*}(\mathfrak{a})$, let $I_{\chi}^{(1)} \subset \mathfrak{Z}(\mathfrak{a})$ be the corresponding ideal (cf. Definition 3.3.1), and $\mu_{\mathcal{U}}\left(I_{\chi}^{(1)}\right) \subset \mathfrak{Z}_{X^{(1)}}$ its image in $\operatorname{Fr} . \mathcal{D}_{X}=\pi \cdot \mathcal{D}^{(1)}$. From commutativity of diagram (3.5.1) for $t=1$ and formula (3.3.3), we deduce that the canonical isomorphism $\mathfrak{Z}_{X^{(1)}} \stackrel{\sim}{\longrightarrow} \pi_{\bullet} \mathcal{O}_{T^{*} X^{(1)}}$ takes the ideal $\mu_{\mathcal{U}}\left(I_{\chi}^{(1)}\right)$ to the ideal $\mu_{\text {alg }}\left(\mathcal{I}_{\varkappa(\chi)}\right) \subset \pi_{\bullet} \mathcal{O}_{T^{*} X^{(1)}}$. On the other hand, it follows from the Poincaré-BirkhoffWitt theorem that the associated graded ideal gr $I_{\chi}^{(1)}$ equals the the augmentation ideal in gr $\mathfrak{Z}(\mathfrak{a})=\operatorname{Sym} \mathfrak{a}^{(1)}$. Thus, specializing diagram (3.5.1) at $t=0$ and $t=1$, respectively, we find

$$
\mu_{\mathcal{U}}\left(I_{\chi}^{(1)}\right)=\mu_{\text {alg }}\left(\mathcal{I}_{\varkappa(\chi)}\right), \quad \text { resp., } \quad \operatorname{gr}\left(\mu_{\mathcal{U}}\left(I_{\chi}^{(1)}\right)\right)=\mu_{\text {alg }}\left(\mathcal{I}_{0}\right) .
$$

We introduce the following subscheme in $T^{*} X^{(1)}$ :

$$
\begin{aligned}
T_{\varkappa(\chi)}^{1, *} & :=\text { zero scheme of } \mu_{\mathcal{U}}\left(I_{\chi}^{(1)}\right)=\text { zero scheme of } \mu_{\text {alg }}\left(\mathcal{I}_{\varkappa(\chi)}\right) \\
& =\left[\mu^{(1)}\right]^{-1}(\varkappa(\chi)),
\end{aligned}
$$

the scheme-theoretic fiber of the moment map $\mu^{(1)}: T^{*} X^{(1)} \rightarrow \mathfrak{a}^{1, *}$ over the point $\varkappa(\chi)$; cf. (3.2.2). For example, if $f: A \rightarrow \mathbb{G}_{\mathbf{m}}$ is an algebraic group character and $\chi:=\operatorname{dlog} f$, then we have $\varkappa(\chi)=0$; see (3.2.4). Hence, $T_{\varkappa(\chi)}^{1, *}=\left[\mu^{-1}(0)\right]^{(1)}$.

3.6. Hamiltonian reduction of differential operators. We keep the setup of subsection 3.5. Thus, we have an Ad $A$-invariant homomorphism $\chi: \mathfrak{a} \rightarrow \mathbb{k}$, the corresponding ideal $I_{\chi}^{(1)} \subset \mathfrak{Z}(\mathfrak{a})$, and its image $\mu_{u}\left(I_{\chi}^{(1)}\right) \subset \pi \cdot \mathcal{D}^{(1)}$ under the map $\mu_{\mathfrak{U}}: \mathcal{U} \mathfrak{a} \rightarrow \pi \cdot \mathcal{D}^{(1)}$, induced by the $A$-action. This image is a central subalgebra in the Azumaya algebra $\mathcal{D}^{(1)}$ on $T^{*} X^{(1)}$, hence, $\mathcal{D}^{(1)} \cdot \pi^{\bullet} \mu_{\mathcal{U}}\left(I_{\chi}^{(1)}\right) \subset \mathcal{D}^{(1)}$ is an $A$-stable two-sided ideal.

We put $\mathcal{D}_{\chi}^{(1)}:=\mathcal{D}^{(1)} / \mathcal{D}^{(1)} \cdot \pi^{\bullet} \mu_{\mathcal{U}}\left(I_{\chi}^{(1)}\right)$. Since $\pi^{\bullet} \mu_{\mathcal{U}}\left(I_{\chi}^{(1)}\right)=\pi^{\bullet} \mu_{\mathrm{alg}}\left(\mathcal{I}_{\varkappa(\chi)}\right)$, by formula (3.5.2), we have

$$
\mathcal{D}_{\chi}^{(1)}=\mathcal{D}^{(1)} / \mathcal{D}^{(1)} \cdot \pi^{\bullet} \mu_{\mathcal{U}}\left(I_{\chi}^{(1)}\right)=\mathcal{D}^{(1)} / \mathcal{D}^{(1)} \cdot \pi^{\bullet} \mu_{\mathrm{alg}}\left(\mathcal{I}_{\varkappa(\chi)}\right)=\left.\mathcal{D}^{(1)}\right|_{T_{\varkappa(\chi)}^{1, *}} .
$$

We see that $\mathcal{D}_{\chi}^{(1)}$ is a restriction of the sheaf $\mathcal{D}^{(1)}$ to $T_{\varkappa(\chi)}^{1, *}$, the scheme-theoretic fiber of the moment map; see (3.5.3). Thus, $\mathcal{D}_{\chi}^{(1)}$ is a coherent sheaf of associative algebras on the subscheme $T_{\varkappa(\chi)}^{1, *}$. By construction, the map $\mathcal{U} \mathfrak{a} \rightarrow \mathcal{D}(X)=$ $\Gamma\left(T^{*} X^{(1)}, \mathcal{D}^{(1)}\right)$ descends, in view of exact sequence (3.3.2), to an $A_{1}$-equivariant algebra homomorphism

$$
\rho_{\chi}: \mathfrak{u}_{\chi}(\mathfrak{a}) \longrightarrow \Gamma\left(T^{*} X^{(1)}, \mathcal{D}_{\chi}^{(1)}\right) .
$$

Recall the two-sided ideal $\mathfrak{i}_{\chi} \subset \mathfrak{u}_{\chi}(\mathfrak{a})$ (see (3.3.4)) and put

$$
\mathcal{E}_{\chi}:=\left(\mathcal{D}_{\chi}^{(1)} / \mathcal{D}_{\chi}^{(1)} \cdot \mathfrak{i}_{\chi}\right)^{A_{1}} \text {. }
$$

This is a sheaf on $T_{\varkappa(\chi)}^{1, *}$ that may be thought of as a Hamiltonian reduction of the algebra $\mathcal{D}_{\chi}^{(1)}$ with respect to the action of $A_{1}$. The construction of subsection 3.4 applied to $\mathrm{D}:=\mathcal{D}_{\chi}^{(1)}$ and to the homomorphism (3.6.2), gives $\varepsilon_{\chi}$ the natural structure of a coherent sheaf of associative algebras on the scheme $T_{\varkappa(\chi)}^{1, *} \subset T^{*} X^{(1)}$. Observe further that the $A$-action on $\mathcal{D}^{(1)}$ factors, when restricted to $A_{1}$-invariants, through the quotient $A^{(1)}=A / A_{1}$. Thus, the sheaf $\mathcal{E}_{\chi}$ acquires an $A^{(1)}$-equivariant structure. 
On the other hand, rather than performing the Hamiltonian reduction of $\mathcal{D}_{\chi}^{(1)}$ with respect to the $A_{1}$-action, one may perform the Hamiltonian reduction of $\mathcal{D}^{(1)}$, a larger object, with respect to the action of $A$, a larger group, that is, to consider $A$-invariants in $\mathcal{D}^{(1)} / \mathcal{D}^{(1)} \cdot I_{\chi}$ (abusing notation, we will write $\mathcal{D}^{(1)} \cdot I_{\chi}$ instead of $\mathcal{D}^{(1)} \cdot \pi^{\bullet} \mu_{\mathcal{U}}\left(I_{\chi}\right)$, and use similar notation in other cases).

The elementary result below is a manifestation of the general principle saying that Hamiltonian reduction can be performed in stages: to make a Hamiltonian reduction with respect to $A$, one can first perform Hamiltonian reduction with respect to $A_{1}$, and then make reduction with respect to $A^{(1)}=A / A_{1}$.

Lemma 3.6.4. There is a canonical algebra isomorphism

$$
\Gamma\left(X, \mathcal{D}_{X} / \mathcal{D}_{X} \cdot I_{\chi}\right)^{A} \cong \Gamma\left(T_{\varkappa(\chi)}^{1, *}, \mathcal{E}_{\chi}\right)^{A^{(1)}} .
$$

Proof. It is clear that, in $\mathcal{D}^{(1)} / \mathcal{D}^{(1)} \cdot I_{\chi}^{(1)}$, one has an equality $\mathcal{D}^{(1)} \cdot I_{\chi} / \mathcal{D}^{(1)} \cdot I_{\chi}^{(1)}=$ $\mathcal{D}_{\chi}^{(1)} \cdot \mathfrak{i}_{\chi}$. Thus, we obtain $\mathcal{D}^{(1)} / \mathcal{D}^{(1)} \cdot I_{\chi} \cong \mathcal{D}_{\chi}^{(1)} / \mathcal{D}_{\chi}^{(1)} \cdot \mathfrak{i}_{\chi}$. Taking $A_{1}$-invariants on both sides, we deduce an isomorphism (of sheaves of associative algebras on $T_{\varkappa(\chi)}^{1, *}$ ):

$$
\left(\mathcal{D}^{(1)} / \mathcal{D}^{(1)} \cdot I_{\chi}\right)^{A_{1}} \cong\left(\mathcal{D}_{\chi}^{(1)} / \mathcal{D}_{\chi}^{(1)} \cdot \mathfrak{i}_{\chi}\right)^{A_{1}}=\varepsilon_{\chi} .
$$

Applying the functor $\Gamma\left(T^{*} X^{(1)},-\right)^{A^{(1)}}$ to $A^{(1)}$-equivariant sheaves in (3.6.5), we obtain a chain of canonical algebra isomorphisms

$$
\begin{aligned}
& \Gamma\left(X, \mathcal{D}_{X} / \mathcal{D}_{X} \cdot I_{\chi}\right)^{A} \cong \Gamma\left(T^{*} X^{(1)}, \mathcal{D}^{(1)} / \mathcal{D}^{(1)} \cdot I_{\chi}\right)^{A} \\
& \cong \Gamma\left(T^{*} X^{(1)},\left(\mathcal{D}^{(1)} / \mathcal{D}^{(1)} \cdot I_{\chi}\right)^{A_{1}}\right)^{A^{(1)}} \quad(\text { by }(3.6 .5)) \\
& \cong \Gamma\left(T^{*} X^{(1)}, \mathcal{E}_{\chi}\right)^{A^{(1)}} \cong \Gamma\left(T_{\varkappa(\chi)}^{1, *}, \mathcal{E}_{\chi}\right)^{A^{(1)}} \text {. }
\end{aligned}
$$

The lemma follows.

3.7. The case of free $A$-action. Keep the notation of 83.6 and let $\chi=0$, hence $I_{\chi}=I_{+} \subset \mathcal{U} \mathfrak{a}$ is the augmentation ideal. Write $\mathcal{D}_{X} \cdot I_{+}=\mathcal{D}_{X} \cdot \mathfrak{a}$ for the left ideal generated by the image of $I_{+}$under the homomorphism $\mu_{\mathfrak{U}}: \mathcal{U} \mathfrak{a} \rightarrow \mathcal{D}(X)$. Applying the construction of subsection 3.4 to the algebra $D:=\mathcal{D}_{X}$ and to the two-sided ideal $I_{+} \subset \mathcal{U} \mathfrak{a}$ one gets an associative algebra $\left(\mathcal{D}_{X} / \mathcal{D}_{X} \cdot I_{+}\right)^{A}$.

Assume now that the $A$-action on $X$ is free and, moreover, there is a smooth variety $Y$, and a smooth universal geometric quotient morphism $\operatorname{pr}_{Y}: X \rightarrow Y$ (whose fibers are exactly the $A$-orbits); see [GIT, Definition 0.7]. It is well known that the algebra of differential operators on $Y$ can be expressed in terms of differential operators on $X$ as follows:

$$
\mathcal{D}_{Y} \cong\left(\left(\operatorname{pr}_{Y}\right) \cdot\left(\mathcal{D}_{X} / \mathcal{D}_{X} \cdot I_{+}\right)\right)^{A}=\left(\left(\operatorname{pr}_{Y}\right) \cdot\left(\mathcal{D}_{X} / \mathcal{D}_{X} \cdot \mu_{\mathcal{U}}(\mathfrak{a})\right)\right)^{A} .
$$

More generally, fix an algebraic homomorphism $\chi: A \rightarrow \mathbb{G}_{\mathbf{m}}$. Given a free $A$-action on $X$, let $\mathcal{O}_{Y}(\chi)$ be an invertible sheaf on $Y$ defined as the subsheaf of $\left(\operatorname{pr}_{Y}\right) \cdot \mathcal{O}_{X}$ formed by the functions $f$ such that $a^{*}(f)=\chi(a) \cdot f, \forall a \in A$. Let $\mathcal{D}_{Y}\left(\mathcal{O}_{Y}(\chi)\right)$ be the corresponding sheaf of twisted differential operators, and $\mathcal{D}(Y, \chi):=\Gamma\left(Y, \mathcal{D}_{Y}\left(\mathcal{O}_{Y}(\chi)\right)\right.$ the algebra of its global sections.

There is a $\chi$-twisted version of formula (3.7.1) that provides a canonical isomorphism $\left(\left(\operatorname{pr}_{Y}\right) .\left(\mathcal{D}_{X} / \mathcal{D}_{X} \cdot I_{\chi}\right)\right)^{A} \stackrel{\sim}{\longrightarrow} \mathcal{D}_{Y}(\chi)$ (isomorphism of sheaves of algebras 
on $Y$ ). Taking global sections on each side of the isomorphism, we get algebra isomorphisms:

$$
\Gamma\left(T_{\varkappa(\chi)}^{1, *}, \mathcal{E}_{\chi}\right)^{A^{(1)}} \underset{\text { Lemma } \sqrt{3.6 .4}}{\sim} \Gamma\left(X, \mathcal{D}_{X} / \mathcal{D}_{X} \cdot I_{\chi}\right)^{A} \cong \mathcal{D}(Y, \chi) .
$$

The isomorphism above makes sense, in effect, not only for $\chi \in \mathbb{X}^{*}(A)$, but also in a slightly more general setting where $\chi \in \mathbb{X}^{*}(\mathfrak{a})$ is an $\operatorname{Ad} A$-invariant Lie algebra character that does not necessarily exponentiate to an algebraic group homomorphism $A \rightarrow \mathbb{G}_{\mathbf{m}}$. Although, generally, the sheaf $\mathcal{O}_{Y}(\chi)$ is not defined in such a case, the corresponding sheaf $\mathcal{D}_{Y}(\chi)$ of twisted differential operators is always well defined (cf. [BB]), and the isomorphism in (3.7.2) still holds.

Remark 3.7.3. The algebra $\mathcal{D}(Y, \chi)$ may be thought of as a quantization of the commutative algebra $\mathbb{k}\left[\mu^{-1}(\chi)\right]^{A}$, the coordinate ring of the Hamiltonian reduction of $T^{*} X$ with respect to the 1 -point orbit $\{\chi\} \subset \mathfrak{a}^{*}$ and the moment map $\mu: T^{*} X \rightarrow$ $\mathfrak{a}^{*}$.

\section{Azumaya algebras via Hamiltonian Reduction}

4.1. The main result. Let $X$ be a smooth $A$-variety. Below, we are going to extend considerations of subsection 3.7 to a more general case where the $A$-action on $X$ is not necessarily free, but the corresponding Hamiltonian $A$-action on $T^{*} X$ is free on an open subset of $T^{*} X$.

There is a natural action of the multiplicative group $\mathbb{G}_{\mathbf{m}}$ on the vector space $\mathfrak{a}^{*}$ and also on the vector bundle $T^{*} X$, by dilations. The moment map $\mu: T^{*} X \rightarrow \mathfrak{a}^{*}$ is clearly compatible with these two actions. It is also compatible with the $A$-actions, and the latter commute with the $\mathbb{G}_{\mathbf{m}}$-actions. Thus, $\mu$ is an equivariant morphism between $\mathbb{G}_{\mathbf{m}} \times A$-varieties.

Let $\mu^{-1}(0) \subset T^{*} X$ be the scheme-theoretic zero fiber of the moment map. This is clearly a $\mathbb{G}_{\mathbf{m}} \times A$-stable subscheme in $T^{*} X$.

From now on, we make the following:

Basic Assumptions 4.1.1. There is a Zariski open $\mathbb{G}_{\mathbf{m}} \times A$-stable subscheme $\mathrm{M} \subset \mu^{-1}(0)$ which is a reduced smooth locally-closed connected subvariety in $T^{*} X$ such that:

- The differential of the moment map $\mu: T^{*} X \rightarrow \mathfrak{a}^{*}$ is surjective at any point of $\mathrm{M}$.

- The $A$-action on $\mathrm{M}$ is free, moreover, there is a smooth variety $\mathcal{M}$ and a smooth universal geometric quotient morphism $\mathrm{M} \rightarrow \mathcal{M}$ (in particular, it is a principal $A$-bundle whose fibers are precisely the $A$-orbits in M) (see GIT]).

- The natural $\mathbb{G}_{\mathbf{m}}$-action on the algebra $\mathbb{k}[\mathcal{M}]$ (arising from the $\mathbb{G}_{\mathbf{m}}$-action on $\mathrm{M}$ ) has no negative weights, more geometrically, the induced $\mathbb{G}_{\mathbf{m}}$-action on the scheme $\mathcal{M}_{\mathrm{aff}}:=\operatorname{Spec} \mathbb{k}[\mathcal{M}]$, the affinization of $\mathcal{M}$, is an attraction.

- The canonical projection $f: \mathcal{M} \rightarrow \mathcal{M}_{\text {aff }}$ is a proper morphism such that $R^{j} f_{*} \mathcal{O}_{\mathcal{M}}=0, \forall j>0$.

The assumptions above insure that the standard symplectic structure on $T^{*} X$ induces a symplectic structure on $\mathcal{M}$. Thus, $\mathcal{M}$ is a symplectic manifold that may be thought of as a Hamiltonian reduction of $T^{*} X$ at 0 . In characteristic zero, this implies the vanishing: $R^{j} f_{*} \mathcal{O}_{\mathcal{M}}=0$ for $j>0$, due to the Grauert-Riemenschneider 
theorem. Such a vanishing is false in characteristic $p$, in general. However, it is known to hold in the important special case of Frobenius split varieties; see [MVK]. This applies, in particular, in the special case of the Hilbert-Chow morphism $f=\Upsilon$ : Hilb $^{n} \mathbb{A}^{2} \rightarrow\left(\mathbb{A}^{2}\right)^{n} / S_{n}$, since the Hilbert scheme Hilb $\mathbb{A}^{2}$ is known to be Frobenius split $[\mathrm{KT}$. The above vanishing also holds, for large enough primes $p$, provided the variety $\mathcal{M}$ is obtained as a reduction mod $p$ of a variety $\mathcal{M}_{\mathbb{Z}}$ defined over the algebraic integers. This may be deduced, by a standard semi-continuity argument (cf. [Har, III, 12.8]) from the corresponding result in characteristic zero.

Now, let $\chi \in \mathbb{X}^{*}(\mathfrak{a})$ be such that $\varkappa(\chi)=0$. Recall the notation $T_{\varkappa(\chi)}^{1, *}:=$ $\left[\mu^{(1)}\right]^{-1}(\varkappa(\chi))$. The equation $\varkappa(\chi)=0$ implies that $T_{\varkappa(\chi)}^{1, *}=\left[\mu^{-1}(0)\right]^{(1)}$, which is clearly a $\mathbb{G}_{\mathbf{m}} \times A^{(1)}$-stable subscheme in $T^{*} X^{(1)}$. Further, by the Basic Assumptions, the scheme $\left[\mu^{-1}(0)\right]^{(1)}$ contains $\mathbf{M}^{(1)}$, the Frobenius twist of $\mathrm{M}$, as an open subscheme. Thus, for any $\chi \in \mathbb{X}^{*}(\mathfrak{a})$ such that $\varkappa(\chi)=0$, the Basic Assumptions yield the following diagram:

$$
T_{\varkappa(\chi)}^{1, *}=\left[\mu^{-1}(0)\right]^{(1)} \longleftrightarrow \mathrm{M}^{(1)} \underset{\text { principal } A^{(1)} \text {-bundle }}{\longleftrightarrow} \mathcal{M}^{(1)} \text {. }
$$

We are going to define a coherent sheaf $\mathcal{A}_{\chi}$ on $\mathcal{M}^{(1)}$ that will be an Azumaya $\mathcal{O}_{\mathcal{N}(1)}$-algebra of degree $p^{1 / 2 \operatorname{dim} \mathcal{N}}$, to be called the quantum Hamiltonian reduction of $\mathcal{D}_{X}$ at $\varkappa(\chi)$. To this end, we restrict $\mathcal{E}_{\chi}=\left(\mathcal{D}_{\chi}^{(1)} / \mathcal{D}_{\chi}^{(1)} \cdot \mathfrak{i}_{\chi}\right)^{A_{1}}$, an $A^{(1)}$-equivariant sheaf on $T_{\varkappa(\chi)}^{1, *} \subset T^{*} X^{(1)}$ (cf. (3.6.3) ), to the open subset $\mathrm{M}^{(1)}$, and consider the push-forward of that restriction under the map $\varpi: \mathrm{M}^{(1)} \longrightarrow \mathcal{M}^{(1)}$; cf. (4.1.2).

Definition 4.1.3. We define the following coherent sheaf of associative algebras on $\mathcal{M}^{(1)}$ :

$$
\mathcal{A}_{\chi}:=\varpi_{\bullet}\left(\left.\mathcal{E}_{\chi}\right|_{\mathrm{M}^{(1)}}\right)^{A^{(1)}}, \text { and put } \mathrm{A}_{\chi}:=\Gamma\left(\mathcal{M}^{(1)}, \mathcal{A}_{\chi}\right)
$$

Assume next that we are given two points $\chi, \psi \in \mathbb{X}^{*}(\mathfrak{a})$, such that the character $\chi-\psi: \mathfrak{a} \rightarrow \mathbb{k}$ can be exponentiated to a group homomorphism $A \rightarrow \mathbb{G}_{\mathbf{m}}$, i.e., such that $\chi-\psi \in \operatorname{dlog}\left(\mathbb{X}^{*}(A)\right)$. Then, formula (3.2.4) yields $\varkappa(\chi)=\varkappa(\psi)$. Hence we have $T_{\varkappa(\chi)}^{1, *}=T_{\varkappa(\psi)}^{1, *}$, and we may view the set $\mathcal{M}$ as a geometric quotient of an open subset of either $T_{\varkappa(\chi)}^{1, *}$ or $T_{\varkappa(\psi)}^{1, *}$.

The result below that will play a key role in subsequent sections, is a generalization of [BK, Proposition 4.8].

Theorem 4.1.4. Let $\chi \in \mathfrak{a}^{*}$ be an $A$-fixed point such that $\varkappa(\chi)=0$ and such that the Basic Assumptions 4.1 .1 hold. Then we have:

(i) The sheaf $\mathcal{A}_{\chi}$ is a sheaf of Azumaya algebras on $\mathcal{M}^{(1)}$ equipped with a canonical algebra morphism $\Xi_{\chi}: \Gamma\left(X, \mathcal{D}_{X} / \mathcal{D}_{X} \cdot I_{\chi}\right)^{A} \longrightarrow \Gamma\left(\mathcal{M}^{(1)}, \mathcal{A}_{\chi}\right)$.

(ii) For all $i>0$, we have $H^{i}\left(\mathcal{M}^{(1)}, \mathcal{A}_{\chi}\right)=0$.

(iii) If the algebra $\mathrm{A}_{\chi}=\Gamma\left(\mathcal{M}^{(1)}, \mathcal{A}_{\chi}\right)$ has finite homological dimension, then the functors below give mutually inverse equivalences of bounded derived categories of sheaves of coherent $\mathcal{A}_{\chi}$-modules and finitely generated $\mathrm{A}_{\chi}$-modules, respectively:

$$
D^{b}\left(\mathcal{A}_{\chi} \text {-Mod }\right) \underset{\mathcal{A}_{\chi} \otimes_{\mathrm{A}_{\chi}} L \longleftarrow L}{\stackrel{\mathcal{L} \longrightarrow \mathrm{R} \Gamma\left(\mathcal{M}^{(1)}, \mathcal{L}\right)}{\longleftarrow}} D^{b}\left(\mathrm{~A}_{\chi}-\operatorname{Mod}\right) .
$$


(iv) Let $\psi \in \mathbb{X}^{*}(\mathfrak{a})$ be another point satisfying all the assumptions above and such that $\chi-\psi \in \operatorname{dlog}\left(\mathbb{X}^{*}(A)\right)$. Then the corresponding Azumaya algebras $\mathcal{A}_{\chi}$ and $\mathcal{A}_{\psi}$ are Morita equivalent (but not necessarily isomorphic).

The rest of this section is devoted to the proof of the theorem.

As will be explained in subsection 4.5 below, part (iii) of Theorem4.1.4 is entirely due to [BK, Proposition 2.2]. A result similar to part (iv) of the theorem is also contained in an updated version of [BMR, §2.3.1].

Remark 4.1.5. (i) The assumption of the theorem that $\varkappa(\chi)=0$ may be relaxed, as will be explained elsewhere.

(ii) We will show, in the course of the proof of Theorem 4.1.4, that the sheaf $\left.\mathcal{E}_{\chi}\right|_{M^{(1)}}$ is also an Azumaya algebra, specifically, we have an Azumaya algebra isomorphism:

$$
\left.\varepsilon_{\chi}\right|_{M^{(1)}}=\varpi^{*} \mathcal{A}_{\chi} .
$$

(iii) It will also follow from the proof that the sheaf $\mathcal{A}_{\chi}$, viewed as a vector bundle on $\mathcal{M}^{(1)}$, is a deformation of the vector bundle $\mathrm{Fr}_{.} \mathcal{O}_{\mathcal{M}}$; in particular, in the Grothendieck group $K\left(\operatorname{Coh}\left(\mathcal{M}^{(1)}\right)\right)$ one has $\left[\mathcal{A}_{\chi}\right]=\left[\operatorname{Fr} . \mathcal{O}_{\mathcal{M}}\right]$. Furthermore, $R^{i} f_{\bullet}^{(1)} \mathcal{A}_{\chi}=0$ for any $i>0$, where $f^{(1)}: \mathcal{M}^{(1)} \rightarrow \mathcal{M}_{\text {aff }}^{(1)}$ denotes the affinization morphism.

4.2. Deformation construction. We are going to apply the Rees algebra formation to all the objects involved in the construction of the algebra $\mathcal{A}_{\chi}$.

In more detail, the standard filtration on $\mathcal{U} \mathfrak{a}$ induces a filtration on the ideal $I_{\chi} \subset \mathcal{U} \mathfrak{a}$, and we form a graded ideal $\mathcal{R e e s} I_{\chi} \subset \mathcal{R}$ ees $\mathcal{U} \mathfrak{a}$, which is generated by the set $\{x-t \cdot \chi(x)\}_{x \in \mathfrak{a}}$. Further, let $\mathcal{R} \mathfrak{Z}(\mathfrak{a})$ be a $\mathbb{k}[t]$-subalgebra in $\mathcal{R}$ ees $\mathcal{U} \mathfrak{a}$ generated by the image of the homomorphism $\mathbf{z}_{\mathcal{R} U}: \operatorname{Sym} \mathfrak{a}^{(1)} \rightarrow \mathcal{R}$ ees $\mathcal{U} \mathfrak{a}$; see (3.1.1). Thus, $\mathcal{R} \mathfrak{Z}(\mathfrak{a}) \subset \mathcal{R}$ ees $\mathcal{U} \mathfrak{a}$ is a graded central subalgebra, and $\mathcal{R} I_{\chi}^{(1)}:=$ $\mathcal{R} \mathfrak{Z}(\mathfrak{a}) \cap \mathcal{R}$ ees $I_{\chi}$ is a graded ideal in $\mathcal{R} \mathfrak{Z}(\mathfrak{a})$. For any $x \in \mathfrak{a}$, we have $x^{p}-t^{p-1} \cdot x^{[p]}$ $-t^{p} \cdot \varkappa(\chi)(x)=\mathbf{z}_{\mathcal{R} \mathcal{U}}(x)-t^{p} \cdot \varkappa(\chi)(x) \in \mathcal{R} \mathfrak{Z}(\mathfrak{a})$. On the other hand, the equations

$$
\begin{aligned}
x^{p}-t^{p-1} \cdot x^{[p]}-t^{p} \cdot \varkappa(\chi)(x)=\left(x^{p}-t^{p} \cdot \chi(x)^{p}\right)-\left(t^{p-1} \cdot x^{[p]}-t^{p} \cdot \chi^{[1]}(x)\right) \\
=(x-t \cdot \chi(x))^{p}-t^{p-1} \cdot\left(x^{[p]}-t \cdot \chi\left(x^{[p]}\right)\right) \in \mathcal{R e e s} I_{\chi}
\end{aligned}
$$

show that $x^{p}-t^{p-1} \cdot x^{[p]}-t^{p} \cdot \varkappa(\chi)(x) \in \mathcal{R} I_{\chi}^{(1)}$. Moreover, it is easy to verify that the elements of this form generate $\mathcal{R} I_{\chi}^{(1)}$ as an ideal.

Next, we apply the Rees algebra construction to the filtered sheaf $\mathrm{Fr} . \mathcal{D}_{X}$. Inside $\operatorname{Fr} . \mathcal{D}_{X}$, we have two left ideals $\operatorname{Fr} . \mathcal{D}_{X} \cdot \mu_{\mathcal{U}}\left(I_{\chi}^{(1)}\right) \subset \operatorname{Fr} . \mathcal{D}_{X} \cdot \mu_{\mathcal{U}}\left(I_{\chi}\right)$, generated by the images of the sets $I_{\chi}^{(1)} \subset I_{\chi} \subset \mathcal{U} \mathfrak{a}$, respectively, under the moment map $\mu_{\mathcal{U}}: \mathcal{U} \mathfrak{a} \rightarrow \operatorname{Fr} . \mathcal{D}_{X}$. The filtration on $\operatorname{Fr} . \mathcal{D}_{X}$ induces by restriction natural filtrations on $\operatorname{Fr} . \mathcal{D}_{X} \cdot \mu_{\mathcal{U}}\left(I_{\chi}^{(1)}\right)$ and on $\operatorname{Fr} . \mathcal{D}_{X} \cdot \mu_{\mathcal{U}}\left(I_{\chi}\right)$. Thus, we obtain graded ideals $\boldsymbol{R}$ ees $\left(\operatorname{Fr} . \mathcal{D}_{X} \cdot \mu_{\mathcal{U}}\left(I_{\chi}^{(1)}\right)\right) \subset \mathcal{R}$ ees $\left(\operatorname{Fr} . \mathcal{D}_{X} \cdot \mu_{\mathcal{U}}\left(I_{\chi}\right)\right) \subset \mathcal{R}$ ees $\left(\operatorname{Fr} . \mathcal{D}_{X}\right)$.

On the other hand, we have a moment map $\mu_{\mathcal{R}}: \mathcal{R}$ ees $\mathcal{U} \mathfrak{a} \rightarrow \mathcal{R}$ ees $\left(\mathrm{Fr} . \mathcal{D}_{X}\right)$, which takes the central subalgebra $\mathcal{R} \mathfrak{Z}(\mathfrak{a}) \subset \mathcal{R}$ ees $\mathcal{U} \mathfrak{a}$ into the central subalgebra $\mathcal{R}$ ees $\mathfrak{Z}_{X^{(1)}} \subset \mathcal{R}$ ees $\left(\mathrm{Fr} . \mathcal{D}_{X}\right)$; cf. diagram (3.5.1). Hence, the image of $\mathcal{R} I_{\chi}^{(1)}$ is the subalgebra $\mu_{\mathcal{R}}\left(\mathcal{R} I_{\chi}^{(1)}\right) \subset \mathcal{R}$ ees $\mathfrak{Z}_{X^{(1)}}$, and we have $\mu_{\mathcal{R}}\left(\mathcal{R} I_{\chi}^{(1)}\right) \cdot \mathcal{R}$ ees $\left(\mathrm{Fr} . \mathcal{D}_{X}\right) \subset$ $\mathcal{R}$ ees $\left(\operatorname{Fr}_{.} \mathcal{D}_{X} \cdot \mu_{\mathcal{U}}\left(I_{\chi}^{(1)}\right)\right)$, where the inclusion is strict, in general. 
Furthermore, we have a $\mathbb{G}_{\mathbf{m}}$-equivariant sheaf $\mathcal{R} \mathcal{D}^{(1)}$ on $\mathbb{A}^{1} \times T^{*} X^{(1)}$ corresponding to the graded algebra $\mathcal{R}$ ees $\left(F r . \mathcal{D}_{X}\right)$. The above constructed graded ideals in the algebra $\mathcal{R}$ ees $\left(\mathrm{Fr} . \mathcal{D}_{X}\right)$ give rise to the following three $\mathbb{G}_{\mathbf{m}}$-equivariant sheaves of left ideals in $\boldsymbol{R} \mathcal{D}^{(1)}$ :

$$
\begin{aligned}
\mathcal{R D}^{(1)} & \cdot\left\{x^{p}-t^{p-1} \cdot x^{[p]}-t^{p} \cdot \varkappa(\chi)(x)\right\}_{x \in \mathfrak{a}}=\mathcal{R} \mathcal{D}^{(1)} \cdot \mathcal{R} I_{\chi}^{(1)} \\
& \subset \mathcal{R}\left(\mathcal{D}^{(1)} \cdot I_{\chi}^{(1)}\right) \subset \mathcal{R}\left(\mathcal{D}^{(1)} \cdot I_{\chi}\right)
\end{aligned}
$$

where we follow our usual convention to drop the symbols $\mu_{\mathcal{U}}$ and $\mu_{\mathcal{R}}$ from the notation.

By $(2.4 .3)$, we have a graded algebra isomorphism $\mathcal{R}$ ees $\mathfrak{Z}_{X^{(1)}} \cong \mathcal{O}_{\mathbb{A}^{1} \times T^{*} X^{(1)}}$. Thus, $\mu_{\mathcal{R}}\left(\mathcal{R} I_{\chi}^{(1)}\right)$ may be viewed as a subset in $\mathcal{O}_{\mathbb{A}^{1} \times T^{*} X^{(1)}}$, and from commutativity of diagram (3.5.1) we deduce that the set $\left\{\mu_{\text {alg }}^{(1)}(x)-t^{p} \cdot \varkappa(\chi)(x)\right\}_{x \in \mathfrak{a}}$ generates the ideal $\mu_{\mathcal{R}}\left(\mathcal{R} I_{\chi}^{(1)}\right) \cdot \mathcal{O}_{\mathbb{A}^{1} \times T^{*} X^{(1)}}$. Thus, in $\mathbb{A}^{1} \times T^{*} X^{(1)}$, we have

(4.2.2) zero-scheme of $\mu_{\mathcal{R}}\left(\mathcal{R} I_{\chi}^{(1)}\right)=\left\{(t, \xi) \in \mathbb{A}^{1} \times T^{*} X^{(1)} \mid \mu^{(1)}(\xi)=t^{p} \cdot \varkappa(\chi)\right\}$.

The sheaf $\mathcal{R} \mathcal{D}^{(1)} / \mathcal{R} \mathcal{D}^{(1)} \cdot \mathcal{R} I_{\chi}^{(1)}$ is clearly supported on the subscheme (4.2.2). We conclude that its quotient $\boldsymbol{R} \mathcal{D}^{(1)} / \mathcal{R}\left(\mathcal{D}^{(1)} \cdot I_{\chi}\right)$ (cf. (4.2.1) ) is supported on the subscheme (4.2.2) as well.

Now let $\chi \in \mathbb{X}^{*}(\mathfrak{a})$ be such that $\varkappa(\chi)=0$. Then the set in 4.2.2) reduces to a direct product $\mathbb{A}^{1} \times\left[\mu^{-1}(0)\right]^{(1)}$. One checks further, going through the identifications used above, that the $\mathbb{G}_{\mathbf{m}} \times A^{(1)}$-action on $\mathbb{A}^{1} \times T_{\varkappa(\chi)}^{1, *}=\mathbb{A}^{1} \times\left[\mu^{-1}(0)\right]^{(1)}$ arising from the natural grading and from the $A^{(1)}$-action on $\mathcal{R} I_{\chi}^{(1)}$, respectively, is the one where the group $\mathbb{G}_{\mathbf{m}}$ acts diagonally, and the group $A^{(1)}$ acts only on the factor $\left[\mu^{-1}(0)\right]^{(1)}$.

Recall the sheaf $\mathcal{R} \mathcal{D}^{(1)} / \mathcal{R}\left(\mathcal{D}^{(1)} \cdot I_{\chi}\right)$, which is supported on $\mathbb{A}^{1} \times\left[\mu^{-1}(0)\right]^{(1)}$, since $\varkappa(\chi)=0$. We restrict this sheaf to the open subscheme $\mathbb{A}^{1} \times \mathrm{M}^{(1)} \subset \mathbb{A}^{1} \times\left[\mu^{-1}(0)\right]^{(1)}$.

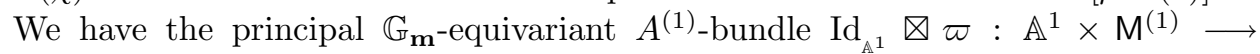
$\mathbb{A}^{1} \times \mathcal{M}^{(1)}$. We define

$$
\mathcal{R} \mathcal{F}:=\left(\operatorname{Id}_{\mathbb{A}^{1}} \otimes \varpi\right) .\left(\mathcal{R} \mathcal{D}^{(1)} / \mathcal{R}\left(\mathcal{D}^{(1)} \cdot I_{\chi}\right)\right) .
$$

This is a $\mathbb{G}_{\mathbf{m}}$-equivariant quasi-coherent sheaf of $A$-modules on $\mathbb{A}^{1} \times \mathcal{M}^{(1)}$ which is flat over the $\mathbb{A}^{1}$-factor. Let $\mathcal{R} \mathcal{F}^{A}$ denote the subsheaf of its $A$-invariant sections.

Lemma 4.2.4. There is a $\mathbb{G}_{\mathbf{m}}$-equivariant sheaf isomorphism $\left.\boldsymbol{R \mathcal { F }}^{A}\right|_{\{0\} \times \mathcal{M}(1)} \cong$ Fr. $\mathcal{O}_{\mathcal{N}}$.

In the course of the proof below, we will repeatedly use the following elementary result.

Lemma 4.2.5. Let $\mathrm{D}$ be a graded algebra, and $M$ a t-torsion free, graded $\mathrm{D}[t]$ module $(\operatorname{deg} t=1)$ such that $M / t M$ is a rank $m$ free, resp. projective, graded $\mathrm{D}$-module. Then, $M$ is a rank $m$ free, resp. projective, $\mathrm{D}[t]$-module.

Proof of Lemma 4.2.4. Recall that every point in any $\mathbb{G}_{\mathbf{m}}$-variety is known to have a $\mathbb{G}_{\mathbf{m}}$-stable affine Zariski open neighborhood. Applying this to the $\mathbb{G}_{\mathbf{m}}$-action on $\mathcal{M}$, we may replace $\mathcal{M}$ by a $\mathbb{G}_{\mathbf{m}}$-stable affine Zariski open subset $\mathcal{Y} \subset \mathcal{M}$. Let $Y$ be the inverse image of $\mathcal{Y}$ under the bundle map $\mathrm{M} \rightarrow \mathcal{M}$. Thus, $Y$ is a $\mathbb{G}_{\mathbf{m}} \times A$-stable affine Zariski open subset in $\mathrm{M}$, and we put

$$
\mathrm{RF}:=\Gamma\left(\mathbb{A}^{1} \times \mathcal{Y}^{(1)}, \mathcal{R} \mathcal{F}\right)=\Gamma\left(\mathbb{A}^{1} \times Y^{(1)}, \mathcal{R} \mathcal{D}^{(1)} / \mathcal{R}\left(\mathcal{D}^{(1)} \cdot I_{\chi}\right)\right) .
$$


Thus, $\mathrm{RF}$ is a graded flat $\mathbb{k}[t]$-module, where $t$ stands for the coordinate on $\mathbb{A}^{1}$.

With these notations, the statement of the lemma amounts to the claim that, for all sufficiently small $\mathbb{G}_{\mathbf{m}}$-stable Zariski open affine subsets $\mathcal{Y} \subset \mathcal{M}$, there is a natural graded space isomorphism

$$
\mathrm{RF}^{A} / t \cdot \mathrm{RF}^{A} \cong \Gamma\left(\mathcal{Y}^{(1)}, \operatorname{Fr} \cdot \mathcal{O}_{\mathcal{Y}}\right)\left(=\mathbb{k}[\mathcal{Y}]=\mathbb{k}[Y]^{A}\right) .
$$

To prove (4.2.6), let $\stackrel{\circ}{*}^{*} X$ be the Zariski open (possibly empty) subset in $T^{*} X$ formed by the points $\xi \in T^{*} X$ such that the differential of $\mu: T^{*} X \rightarrow \mathfrak{a}^{*}$ is surjective at $\xi$. Further, let $\mathcal{J} \subset \operatorname{Sym} \mathfrak{a}=\mathbb{k}\left[\mathfrak{a}^{*}\right]$ denote the augmentation ideal. It is clear that $\mu^{-1}(0)$ is the zero scheme of the ideal $\mathcal{O}_{T^{*} X} \cdot \mu_{\text {alg }}(\mathcal{J}) \subset \mathcal{O}_{T^{*} X}$, and that this ideal is reduced at any point of $\mu^{-1}(0) \cap \stackrel{\circ}{T}^{*} X$. It follows, since $\operatorname{gr} I_{\chi}=\mathcal{J}$, that on the Frobenius twist of $\stackrel{\circ}{*}^{*} X$, one has:

$$
\begin{aligned}
\left.\operatorname{gr}\left(\operatorname{Fr} .\left(\mathcal{D}_{X} \cdot I_{\chi}\right)\right)\right|_{T^{\circ} X^{(1)}} & =\left.\left(\left(\operatorname{gr} F r \cdot \mathcal{D}_{X}\right) \cdot\left(\operatorname{gr} F r . I_{\chi}\right)\right)\right|_{T^{*} X^{(1)}} \\
& =\left.\operatorname{Fr} \cdot\left(\mathcal{O}_{T^{*} X} \cdot \mu_{\text {alg }}(\mathcal{J})\right)\right|_{T^{\circ} X^{(1)}},
\end{aligned}
$$

where we identify $\operatorname{gr}\left(\operatorname{Fr} \cdot \mathcal{D}_{X}\right)$ with the corresponding $\mathbb{G}_{\mathbf{m}}$-equivariant sheaf on $T^{*} X^{(1)}$.

We now use our Basic Assumptions saying that the differential of $\mu$ is surjective at any point of the open subset $\mathrm{M} \subset \mu^{-1}(0)$. Hence, $\mathrm{M} \subset T^{*} X \cap \mu^{-1}(0)$ is a nonempty Zariski-open subset in $\mu^{-1}(0)$. Since the sheaf $\operatorname{gr}\left(\operatorname{Fr} . \mathcal{D}_{X}\right) / \operatorname{gr}\left(\operatorname{Fr} . \mathcal{D}_{X} \cdot I_{\chi}\right)$ is supported on $\left[\mu^{-1}(0)\right]^{(1)}$, we obtain

$$
\begin{aligned}
\left.\left(\operatorname{gr}\left(\operatorname{Fr} . \mathcal{D}_{X}\right) / \operatorname{gr}\left(\operatorname{Fr} \cdot \mathcal{D}_{X} \cdot I_{\chi}\right)\right)\right|_{\mathrm{M}^{(1)}} \\
=\left.\left(\operatorname{gr}\left(\operatorname{Fr} \cdot \mathcal{D}_{X}\right) / \operatorname{gr}\left(\operatorname{Fr} \cdot \mathcal{D}_{X} \cdot I_{\chi}\right)\right)\right|_{T^{*} X^{(1)} \cap M^{(1)}} \\
=\left.\left(\left(\operatorname{Fr} \cdot \mathcal{O}_{T^{*} X}\right) / \operatorname{Fr} \cdot\left(\mathcal{O}_{T^{*} X} \cdot \mu_{\mathrm{alg}}(\mathcal{J})\right)\right)\right|_{T^{*} X^{(1)} \cap \mathrm{M}^{(1)}} \\
\quad=\operatorname{Fr} \cdot\left(\left.\mathcal{O}_{T^{*} X}\right|_{T^{*} X \cap \mathrm{M}}\right)=\operatorname{Fr} \cdot \mathcal{O}_{\mathrm{M}} .
\end{aligned}
$$

The definition of Rees algebra implies readily (see (2.4.1) and (2.5.3)), that the restriction to $\{0\} \times \mathrm{M}^{(1)}$ of the sheaf $\mathcal{R} \mathcal{D}^{(1)} / \mathcal{R} \mathcal{D}^{(1)} \cdot \mathcal{R} I_{\chi}^{(1)}$ is isomorphic to $\left.\left(\operatorname{gr}\left(\operatorname{Fr} \cdot \mathcal{D}_{X}\right) / \operatorname{gr}\left(\operatorname{Fr} \cdot \mathcal{D}_{X} \cdot I_{\chi}\right)\right)\right|_{M^{(1)}}$, the sheaf in the top line of (4.2.7). Hence, equations (4.2.7) and a flat base change yield $\left.\mathcal{R} \mathcal{F}\right|_{\{0\} \times \mathcal{M}^{(1)}} \cong \varpi$. Fr. $\mathcal{O}_{\mathrm{M}}$. Restricting this sheaf isomorphism to our affine open subset $\mathcal{Y}^{(1)}$ and taking global sections, we obtain canonical graded space isomorphisms

$$
\begin{aligned}
\mathrm{RF} / t \cdot \mathrm{RF} \cong \Gamma\left(\mathcal{Y}^{(1)},\left.\mathcal{R} \mathcal{F}\right|_{\{0\} \times \mathcal{Y}^{(1)}}\right) & \cong \Gamma\left(\mathcal{Y}^{(1)}, \varpi \cdot \operatorname{Fr} \cdot \mathcal{O}_{Y}\right) \\
& \cong \Gamma\left(Y^{(1)}, \operatorname{Fr} \cdot \mathcal{O}_{Y}\right)=\mathbb{k}[Y] .
\end{aligned}
$$

We conclude, comparing the above isomorphisms with those in (4.2.6), that proving the lemma reduces to the following result: The canonical map below gives, for $\mathcal{Y}$ sufficiently small, an isomorphism

$$
\mathrm{RF}^{A} / t \cdot \mathrm{RF}^{A} \stackrel{\sim}{\longrightarrow}(\mathrm{RF} / t \cdot \mathrm{RF})^{A} .
$$

To prove this, we may assume, shrinking $\mathcal{Y}$ if necessary, that there is an étale map $\theta: \widetilde{\mathcal{Y}} \rightarrow \mathcal{Y}$ such that the principal $A$-bundle $Y \rightarrow \mathcal{Y}$ becomes trivial after pullback via $\theta$, i.e., that there is an $\mathbb{G}_{\mathbf{m}} \times A$-equivariant isomorphism $Y \times \mathcal{Y} \widetilde{\mathcal{Y}} \cong A \times \widetilde{\mathcal{Y}}$. 
Using étale base change for the Cartesian square

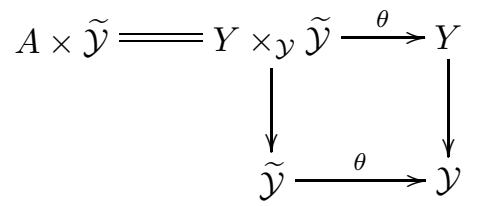

we obtain $\mathbb{G}_{\mathbf{m}} \times A$-equivariant graded algebra isomorphisms

$$
\mathbb{k}[\tilde{Y}]=\mathbb{k}[\tilde{\mathcal{Y}}] \otimes_{\mathbb{k}}[\mathcal{Y}] \mathbb{k}[Y] \cong \mathbb{k}[Y \times \mathcal{Y} \tilde{\mathcal{Y}}] \cong \mathbb{k}[A] \otimes \mathbb{k}[\tilde{\mathcal{Y}}] .
$$

In the leftmost term of this formula, we have used the notation $\tilde{Y}:=Y \times \mathcal{Y} \tilde{\mathcal{Y}}$, and in the rightmost term of the formula, the group $A$ acts trivially on the factor $\mathbb{k}[\widetilde{\mathcal{Y}}]$.

Next, we set

$$
\begin{aligned}
\widetilde{\mathrm{RF}} & :=\Gamma\left(\mathbb{A}^{1} \times \widetilde{Y}^{(1)},\left.\left(\operatorname{Id}_{\mathbb{A}^{1}} \times \theta^{(1)}\right)^{*}\left(\mathcal{R} \mathcal{D}^{(1)} / \mathcal{R}\left(\mathcal{D}^{(1)} \cdot I_{\chi}\right)\right)\right|_{\mathbb{A}^{1} \times Y^{(1)}}\right) \\
& =\mathbb{k}\left[\mathbb{A}^{1} \times \widetilde{Y}^{(1)}\right] \otimes_{\mathbb{k}_{[}\left[\mathbb{A}^{1} \times \mathcal{Y}(1)\right.} \operatorname{RF} .
\end{aligned}
$$

Since $\mathbb{k}\left[\tilde{Y}^{(1)}\right]$ is flat over $\mathbb{k}\left[Y^{(1)}\right]$, from (4.2.8) and (4.2.10) we find

$\widetilde{\mathrm{RF}} / t \cdot \widetilde{\mathrm{RF}}=\mathbb{k}\left[\widetilde{Y}^{(1)}\right] \otimes_{\mathbb{k}\left[\mathcal{Y}^{(1)}\right]}(\mathrm{RF} / t \cdot \mathrm{RF})=\mathbb{k}\left[\widetilde{Y}^{(1)}\right] \otimes_{\mathbb{k}\left[\mathcal{Y}^{(1)}\right]} \mathbb{k}[Y]=\mathbb{k}[\widetilde{Y}] \cong \mathbb{k}[A] \otimes \mathbb{k}[\widetilde{\mathcal{Y}}]$.

Thus, we see that $\widetilde{\mathrm{RF}} / t \cdot \widetilde{\mathrm{RF}}$ is a rank one free $\mathbb{k}[A] \otimes \mathbb{k}[\widetilde{\mathcal{Y}}]$-module. We deduce from Lemma 4.2.5 that $\widetilde{R F}$ is isomorphic to a rank one free $\mathbb{k}[A] \otimes \mathbb{k}\left[\mathbb{A}^{1} \times \widetilde{\mathcal{Y}}\right]$ module. Furthermore, it is easy to show that this isomorphism can be chosen to be $A$-equivariant.

Now, the functor $M \longmapsto M^{A}$, of $A$-invariants, takes short exact sequences of $A$-modules of the form $\mathbb{k}[A] \otimes E$ to short exact sequences. Hence applying this functor to the short exact sequence

$$
0 \longrightarrow \widetilde{\mathrm{RF}} \stackrel{t}{\longrightarrow} \widetilde{\mathrm{RF}} \longrightarrow \widetilde{\mathrm{RF}} / t \cdot \widetilde{\mathrm{RF}} \longrightarrow 0
$$

we deduce the isomorphism

$$
\widetilde{\mathrm{RF}}^{A} / t \cdot \widetilde{\mathrm{RF}}^{A} \stackrel{\sim}{\longrightarrow}(\widetilde{\mathrm{RF}} / t \cdot \widetilde{\mathrm{RF}})^{A} .
$$

The latter isomorphism yields (4.2.9) since the morphism $\widetilde{Y}^{(1)} \rightarrow Y^{(1)}$ is faithfully flat. The lemma is proved.

4.3. Deformation of the algebra $\mathcal{A}_{\chi}$. We mimick formulas (3.6.1) and (3.6.3) and put

$$
\begin{aligned}
\mathcal{R} \mathcal{D}_{\chi}^{(1)} & :=\mathcal{R} \mathcal{D}^{(1)} / \mathcal{R}\left(\mathcal{D}^{(1)} \cdot I_{\chi}^{(1)}\right), \quad \text { and } \\
& \mathcal{R} \varepsilon_{\chi}:=\left(\mathcal{R} \mathcal{D}^{(1)} / \mathcal{R}\left(\mathcal{D}^{(1)} \cdot I_{\chi}\right)\right)^{A_{1}} \cong\left(\mathcal{R} \mathcal{D}_{\chi}^{(1)} / \mathcal{R}\left(\mathcal{D}_{\chi}^{(1)} \cdot \mathfrak{i}_{\chi}\right)\right)^{A_{1}} .
\end{aligned}
$$

The sheaves $\mathcal{R} \mathcal{D}_{\chi}^{(1)}$ and $\mathcal{R} \varepsilon_{\chi}$ are both supported on $\mathbb{A}^{1} \times\left[\mu^{-1}(0)\right]^{(1)}$; see (4.2.2). Following the same strategy that has been used in the construction of the sheaf $\mathcal{A}_{\chi}$, we define

$$
\mathcal{R} \mathcal{A}_{\chi}:=\varpi .\left(\left.\mathcal{R} \varepsilon_{\chi}\right|_{\mathbb{A}^{1} \times \mathrm{M}^{(1)}}\right)^{A^{(1)}} .
$$

This is a $\mathbb{G}_{\mathbf{m}}$-equivariant sheaf of associative algebras on $\mathbb{A}^{1} \times \mathcal{M}^{(1)}$, viewed as a $\mathbb{G}_{\mathbf{m}}$-variety with diagonal action. Write $\mathrm{pr}_{2}: \mathbb{A}^{1} \times \mathcal{M}^{(1)} \rightarrow \mathcal{M}^{(1)}$ for the second projection. 
Lemma 4.3.2. The sheaf $\mathcal{R} \mathcal{A}_{\chi}$ is flat over the $\mathbb{A}^{1}$-factor, and we have

$$
\left.\mathcal{R} \mathcal{A}_{\chi}\right|_{\{0\} \times \mathcal{M}^{(1)}} \cong \operatorname{Fr} \cdot \mathcal{O}_{\mathcal{M}}, \text { and }\left.\mathcal{R} \mathcal{A}_{\chi}\right|_{\left(\mathbb{A}^{1} \backslash\{0\}\right) \times \mathcal{M}^{(1)}} \cong \operatorname{pr}_{2}^{*} \mathcal{A}_{\chi}
$$

Proof. Both the flatness statement and the isomorphism on the right are immediate from the corresponding properties of the Rees algebra.

It remains to study the restriction of the sheaf $\mathcal{R} \mathcal{A}_{\chi}$ to the special divisor $\{0\} \times$ $\mathcal{M}^{(1)}$. From the definition, we find

$$
\begin{aligned}
\left.\left.\mathcal{R} \mathcal{A}_{\chi}\right|_{\{0\} \times \mathcal{M}(1)} \cong\left(\left(\operatorname{Id}_{\mathbb{A}^{1}} \nabla \varpi\right) \cdot \mathcal{R} \varepsilon_{\chi}\right)^{A^{(1)}}\right|_{\{0\} \times \mathcal{M}^{(1)}} \\
\left.\cong\left(\operatorname{Id}_{\mathbb{A}^{1}} \nabla \varpi\right) \cdot\left(\mathcal{R} \mathcal{D}^{(1)} / \mathcal{R}\left(\mathcal{D}^{(1)} \cdot I_{\chi}\right)^{A_{1}}\right)^{A^{(1)}}\right|_{\{0\} \times \mathcal{M}^{(1)}} \\
\left.\cong\left(\operatorname{Id}_{\mathbb{A}^{1}} \nabla \varpi\right) \cdot\left(\mathcal{R} \mathcal{D}^{(1)} / \mathcal{R}\left(\mathcal{D}^{(1)} \cdot I_{\chi}\right)\right)^{A}\right|_{\{0\} \times \mathcal{M}^{(1)}}=\left.\mathcal{R} \mathcal{F}^{A}\right|_{\{0\} \times \mathcal{M}^{(1)}}
\end{aligned}
$$

But $\left.\mathcal{R F}^{A}\right|_{\{0\} \times \mathcal{M}^{(1)}} \cong \mathrm{Fr}_{\text {. }} \mathcal{O}_{\mathcal{M}}$ by Lemma 4.2.4 and we are done.

Next, we set $\mathbf{R}:=\Gamma\left(\mathbb{A}^{1} \times \mathcal{M}^{(1)}, \mathcal{R} \mathcal{A}_{\chi}\right)$, a graded flat $\mathbb{k}[t]$-algebra such that $\mathbf{R} /(t-1) \mathbf{R}=\Gamma\left(\mathcal{N}^{(1)}, \mathcal{A}_{\chi}\right)$. Applying formula (2.4.2) to the algebra $\mathcal{R}:=\mathbf{R}$ and using Lemma 4.3.2, we get a natural increasing filtration on the algebra $\Gamma\left(\mathcal{M}^{(1)}, \mathcal{A}_{\chi}\right)$ such that for the associated graded algebra, to be denoted gr ${ }^{\mathcal{R}} \Gamma\left(\mathcal{M}^{(1)}, \mathcal{A}_{\chi}\right)$, we have $\operatorname{gr}^{\mathcal{R}} \Gamma\left(\mathcal{M}^{(1)}, \mathcal{A}_{\chi}\right)=\mathbf{R} / t \mathbf{R}$. On the other hand, the $\mathbb{G}_{\mathbf{m}}$-action induces a grading on the algebra $\Gamma\left(\mathcal{N}, \mathcal{O}_{\mathcal{M}}\right)=\Gamma\left(\mathcal{M}^{(1)}, \operatorname{Fr}_{.} \mathcal{O}_{\mathcal{M}}\right)$.

Recall the affinization morphism $f: \mathcal{M} \rightarrow \mathcal{M}_{\text {aff }}$. Given $\xi \in \mathcal{M}_{\text {aff }}^{(1)}$, let $\mathcal{M}_{\xi}^{(1)}:=$ $\left[f^{(1)}\right]^{-1}(\xi) \subset \mathcal{M}^{(1)}$ be the fiber of $f^{(1)}: \mathcal{M}^{(1)} \rightarrow \mathcal{M}_{\text {aff }}^{(1)}$ over $\xi$.

Proposition 4.3.3. (i) The sheaf $\mathcal{A}_{\chi}$ is locally free, and $H^{i}\left(\mathcal{M}^{(1)}, \mathcal{A}_{\chi}\right)=0$ for all $i>0$.

(ii) There is a graded algebra isomorphism

$$
\operatorname{gr}^{\mathcal{R}} \Gamma\left(\mathcal{M}^{(1)}, \mathcal{A}_{\chi}\right) \cong \Gamma\left(\mathcal{M}, \mathcal{O}_{\mathcal{M}}\right) \text {. }
$$

(iii) For any $q>0$ we have $R^{q} f_{*}^{(1)} \mathcal{A}_{\chi}=0$ and $H^{q}\left(\mathcal{M}_{\xi}^{(1)}, \mathcal{A}_{\chi}\right)=0, \forall \xi \in \mathcal{M}_{\text {aff }}^{(1)}$.

Proof. We consider the following diagram:

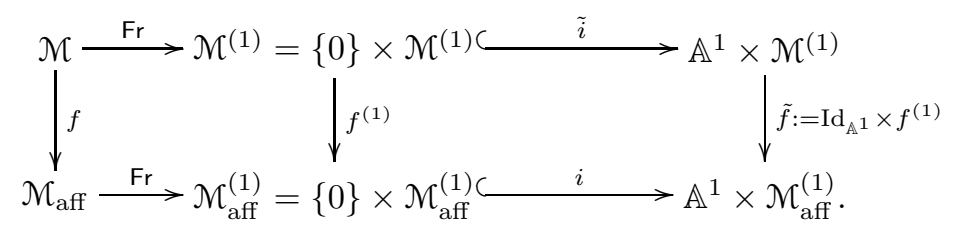

Lemma 4.3 .2 says that $\tilde{i}^{*} \mathcal{R} \mathcal{A}_{\chi} \cong$ Fr. $\mathcal{O}_{\mathcal{M}}$. Thus, $\mathcal{R} \mathcal{A}_{\chi}$ is a $\mathbb{G}_{\mathbf{m}}$-equivariant sheaf on $\mathbb{A}^{1} \times \mathcal{M}^{(1)}$ such that its restriction to the subvariety $\{0\} \times \mathcal{M}^{(1)}$ is a locally free sheaf. It follows that the sheaf $\mathcal{R} \mathcal{A}_{\chi}$ must be locally free. Indeed, every point in $\mathcal{M}^{(1)}$ has a $\mathbb{G}_{\mathbf{m}}$-stable affine Zariski open neighborhood. Taking global sections of $\mathcal{R} \mathcal{A}_{\chi}$ over such a neighborhood, we see that our claim reduces to Lemma 4.2.5. Thus, we have proved that the sheaf $\mathcal{R} \mathcal{A}_{\chi}$, hence its restriction to $\{1\} \times \mathcal{M}^{(1)}$, is a locally free sheaf. But $\left.\mathcal{R} \mathcal{A}_{\chi}\right|_{\{1\} \times \mathcal{M}^{(1)}}=\mathcal{A}_{\chi}$, thus, the first claim of part (i) of the lemma is proved.

Recall further that the variety $\mathcal{M}$ is symplectic, hence it has trivial canonical bundle. Therefore, by our basic assumptions, the higher direct image sheaves $R^{q} f_{*} \mathcal{O}_{\mathcal{M}}$, 
vanish for all $q>0$. Therefore, from the commutative square on the left of (4.3.4) we deduce

$$
R^{q} f_{*}^{(1)}\left(\tilde{i}^{*} \mathcal{R} \mathcal{A}_{\chi}\right)=R^{q} f_{*}^{(1)} \operatorname{Fr} . \mathcal{O}_{\mathcal{M}}=0, \text { for all } \quad q>0
$$

We are going to use (4.3.5) to prove part (iii) of the proposition. To this end, let $\widehat{D}:=\operatorname{Spec} \mathbb{k}[[t]] \subset \mathbb{A}^{1}$ denote the completion of the line $\mathbb{A}^{1}$ at the origin. Thus, $\widehat{D} \times \mathcal{M}^{(1)}$, resp., $\widehat{D} \times \mathcal{M}_{\text {aff }}^{(1)}$, is the formal completion of the scheme $\mathbb{A}^{1} \times \mathcal{M}^{(1)}$ along the closed subscheme $\{0\} \times \mathcal{M}^{(1)}$, resp., formal completion of $\mathbb{A}^{1} \times \mathcal{M}_{\text {aff }}^{(1)}$ along the subscheme $\{0\} \times \mathcal{M}_{\text {aff }}^{(1)}$. We have $\widehat{D} \times \mathcal{M}^{(1)}=\tilde{f}^{-1}\left(\widehat{D} \times \mathcal{M}_{\text {aff }}^{(1)}\right)$, and the restriction of $\tilde{f}$ gives a morphism $\widehat{f}: \widehat{D} \times \mathcal{M}^{(1)} \longrightarrow \widehat{D} \times \mathcal{M}_{\text {aff }}^{(1)}$.

Let $\left.\mathcal{R} \mathcal{A}_{\chi}\right|_{\widehat{D} \times \mathcal{M}^{(1)}}$ denote the restriction of $\mathcal{R} \mathcal{A}_{\chi}$ to $\widehat{D} \times \mathcal{M}^{(1)}$. We will view $\left.\mathcal{R} \mathcal{A}_{\chi}\right|_{\widehat{D} \times \mathcal{M}^{(1)}}$ as a sheaf of abelian groups on the closed fiber $\mathcal{M}^{(1)}=\{0\} \times \mathcal{M}^{(1)}$. This sheaf is clearly isomorphic to an inverse limit of sheaves which are iterated extensions of the sheaf $\tilde{i}^{*} \boldsymbol{\mathcal { R }} \mathcal{A}_{\chi}=\operatorname{Fr} \cdot \mathcal{O}_{\mathcal{M}}$. Hence, formula (4.3.5) implies that

$$
R^{q} \widehat{f}_{*}\left(\left.\mathcal{R} \mathcal{A}_{\chi}\right|_{\widehat{D} \times \mathcal{M}^{(1)}}\right)=0, \text { for all } q>0
$$

On the other hand, let $\widehat{i}: \widehat{D} \times \mathcal{M}_{\text {aff }}^{(1)} \hookrightarrow \mathbb{A}^{1} \times \mathcal{M}_{\text {aff }}^{(1)}$ denote the imbedding. For any $q=0,1, \ldots$, one has $\widehat{i}^{*} R^{q} \tilde{f}_{*} \mathcal{R} \mathcal{A}_{\chi}=R^{q} \widehat{f}_{*}\left(\left.\mathcal{R} \mathcal{A}_{\chi}\right|_{\widehat{D} \times \mathcal{M}(1)}\right)$, by the Formal Functions Theorem; cf. [Har, III, 11.1] or [EG, III, Sect. 4]. Thus, we have proved

$$
\widehat{i}^{*} R^{q} \tilde{f}_{*} \mathcal{R} \mathcal{A}_{\chi}=R^{q} \widehat{f}_{*}\left(\left.\mathcal{R} \mathcal{A}_{\chi}\right|_{\widehat{D} \times \mathcal{M}(1)}\right)=0, \text { for all } q>0 .
$$

Observe next that, for each $q$, the sheaf $R^{q} \tilde{f}_{*} \mathcal{R} \mathcal{A}_{\chi}$ is a $\mathbb{G}_{\mathbf{m}}$-equivariant coherent sheaf on $\mathbb{A}^{1} \times \mathcal{M}_{\text {aff }}^{(1)}$. Therefore, the support of $R^{q} \tilde{f}_{*} \mathcal{R} \mathcal{A}_{\chi}$ is a $\mathbb{G}_{\mathbf{m}}$-stable closed subscheme in $\mathbb{A}^{1} \times \mathcal{M}_{\text {aff }}^{(1)}$. The assumption that the $\mathbb{G}_{\mathbf{m}}$-action on $\mathcal{M}$ be attracting implies that any nonempty $\mathbb{G}_{\mathbf{m}}$-stable closed subscheme in $\mathbb{A}^{1} \times \mathcal{M}_{\text {aff }}^{(1)}$ has a nonempty intersection with $\{0\} \times \mathcal{M}_{\text {aff }}^{(1)}$. Hence, if the sheaf $R^{q} \tilde{f}_{*} \mathcal{R} \mathcal{A}_{\chi}$ is nonzero, it must have a nonzero restriction to the subscheme $\{0\} \times \mathcal{M}_{\text {aff }}^{(1)}$. Since $\{0\} \times \mathcal{M}_{\text {aff }}^{(1)} \subset \widehat{D} \times \mathcal{M}_{\text {aff }}^{(1)}$, this would yield, in particular, that $\widehat{i}^{*} R^{q} \tilde{f}_{*} \mathcal{R} \mathcal{A}_{\chi} \neq 0$, contradicting (4.3.6). Part (iii) of the Proposition follows.

Taking global sections over the affine open set $\left(\mathbb{A}^{1} \backslash\{0\}\right) \times \mathcal{M}_{\text {aff }}^{(1)}$, from the vanishing result of part (iii) and the second isomorphism of Lemma 4.3.2, for any $q>0$, we find

$$
\begin{aligned}
0=\Gamma\left(\left(\mathbb{A}^{1} \backslash\{0\}\right)\right. & \left.\times \mathcal{M}_{\mathrm{aff}}^{(1)}, R^{q} \tilde{f}_{*} \mathcal{R} \mathcal{A}_{\chi}\right)=H^{q}\left(\left(\mathbb{A}^{1} \backslash\{0\}\right) \times \mathcal{M}^{(1)}, \mathcal{R} \mathcal{A}_{\chi}\right) \\
& =H^{q}\left(\left(\mathbb{A}^{1} \backslash\{0\}\right) \times \mathcal{M}^{(1)}, \operatorname{pr}_{2}^{*} \mathcal{A}_{\chi}\right)=\mathbb{k}\left[t, t^{-1}\right] \otimes H^{q}\left(\mathcal{M}^{(1)}, \mathcal{A}_{\chi}\right) .
\end{aligned}
$$

This completes the proof of part (i) of the proposition.

Now, the (ordinary) direct image sheaf $\tilde{f}_{*} \mathcal{R} \mathcal{A}_{\chi}$ is by construction flat over $\mathbb{A}^{1}$, hence, $L^{q} i^{*}\left(\tilde{f}_{*} \mathcal{R} \mathcal{A}_{\chi}\right)=0$ for all $q>0$. Therefore, the vanishing of the higher direct images implies that the Proper Base Change theorem for the Cartesian square on the right of diagram (4.3.4) involves no higher derived functors. Thus, using Base Change and the definition of affinization we obtain

$$
i^{*} \tilde{f}_{*} \mathcal{R} \mathcal{A}_{\chi}=f_{*}^{(1)} \tilde{i}^{*} \mathcal{R} \mathcal{A}_{\chi}=f_{*}^{(1)} \operatorname{Fr}_{*} \mathcal{O}_{\mathcal{M}}=\text { Fr. } f_{*} \mathcal{O}_{\mathcal{M}}=\text { Fr. } \mathcal{O}_{\mathcal{M}} \text { aff }
$$


Observe that we have $\Gamma\left(\mathbb{A}^{1} \times \mathcal{M}_{\text {aff }}^{(1)}, \tilde{f}_{*} \mathcal{R} \mathcal{A}_{\chi}\right)=\Gamma\left(\mathbb{A}^{1} \times \mathcal{M}^{(1)}, \mathcal{R} \mathcal{A}_{\chi}\right)=\mathbf{R}$, the graded $\mathbb{k}[t]$-algebra involved in the definition of filtration on $\Gamma\left(\mathcal{M}^{(1)}, \mathcal{A}_{\chi}\right)$. Therefore, applying the global sections functor $\Gamma\left(\mathbb{A}^{1} \times \mathcal{M}_{\text {aff }}^{(1)},-\right)$ to both sides in (4.3.7) we obtain

$$
\operatorname{gr}^{\mathcal{R}} \Gamma\left(\mathcal{M}^{(1)}, \mathcal{A}_{\chi}\right)=\mathbf{R} / t \mathbf{R} \cong \Gamma\left(\mathcal{M}_{\mathrm{aff}}^{(1)}, \operatorname{Fr} \cdot \mathcal{O}_{\mathcal{M}_{\mathrm{aff}}}\right)=\Gamma\left(\mathcal{M}^{(1)}, \operatorname{Fr} \cdot \mathcal{O}_{\mathcal{M}}\right)=\Gamma\left(\mathcal{M}, \mathcal{O}_{\mathcal{M}}\right)
$$

This proves part (ii) of the proposition.

4.4. Comparison of characters. Fix two characters $\chi, \psi$ such that $\chi-\psi \in$ $\operatorname{dlog} \mathbb{X}^{*}(A)$. Let $\mathcal{O}_{\mathcal{M}}(\psi-\chi)$ be the $(\psi-\chi)$-weight subsheaf in the push-forward of $\mathcal{O}_{\mathrm{M}}$ under the bundle map $\mathrm{M} \rightarrow \mathcal{M}$. The $(\psi-\chi)$-isotypic component of the regular representation $\mathbb{k}[A]$ being 1 -dimensional, we conclude that $\mathcal{O}_{\mathcal{M}}(\psi-\chi)$ is a rank 1 locally free sheaf on $\mathcal{M}$.

Let $\mathcal{D}_{M}^{(1)}$ denote the restriction of the Azumaya algebra $\mathcal{D}^{(1)}$ to the subset $\mathrm{M}^{(1)} \subset$ $T_{\varkappa(\chi)}^{1, *}=T_{\varkappa(\psi)}^{1, *}$. We put $\mathcal{\varepsilon}_{\psi}:=\operatorname{Hom}_{\mathcal{D}_{\mathrm{M}}^{(1)}}\left(\mathcal{D}_{\mathrm{M}}^{(1)} / \mathcal{D}_{\mathbf{M}}^{(1)} \cdot \mathfrak{i}_{\chi}, \mathcal{D}_{\mathbf{M}}^{(1)} / \mathcal{D}_{\mathbf{M}}^{(1)} \cdot \mathfrak{i}_{\psi}\right)$. This is an $A^{(1)}$-equivariant sheaf on $\mathrm{M}^{(1)}$. Therefore, $\varpi \cdot\left({ }_{\chi} \varepsilon_{\psi}\right)$ is a sheaf on $\mathcal{M}^{(1)}$ with fiberwise $A^{(1)}$-action. Let ${ }_{\chi} \mathcal{A}_{\psi}$ be the $(\psi-\chi)$-weight component of $\varpi \cdot\left({ }_{\chi} \mathcal{E}_{\psi}\right)$. It is clear that ${ }_{\chi} \mathcal{A}_{\psi}$ is a coherent sheaf of $\mathcal{A}_{\chi}-\mathcal{A}_{\psi}$-bimodules.

Next, we mimic the argument in subsection 4.2 and observe that the standard increasing filtration on $\varpi . \mathcal{D}_{M}^{(1)}$ induces a natural increasing filtration on $\varpi$. $\left({ }_{\chi} \varepsilon_{\psi}\right)$. Therefore, using the Rees algebra construction, we may form a $\mathbb{G}_{\mathbf{m}}$-equivariant sheaf $\mathcal{R}\left({ }_{\chi} \mathcal{E}_{\psi}\right)$ on $\mathbb{A}^{1} \times \mathrm{M}^{(1)}$. This gives an increasing filtration on $\Gamma\left(\mathcal{M}^{(1)},{ }_{\chi} \mathcal{A}_{\psi}\right)$, an $\mathrm{A}_{\chi}-\mathrm{A}_{\psi}$-bimodule, and we write $\operatorname{gr}^{\mathcal{R}} \Gamma\left(\mathcal{M}^{(1)},{ }^{\mathcal{A}} \mathcal{A}_{\psi}\right)$ for the associated graded $\left(\operatorname{gr} \mathrm{A}_{\chi}\right)$ - $\left(\operatorname{gr} \mathrm{A}_{\psi}\right)$-bimodule.

Lemma 4.4.1. (i) The sheaf $\chi_{\chi} \mathcal{A}_{\psi}$ is locally free, moreover, we have a natural $\mathcal{E}_{\chi}-\mathcal{E}_{\psi}$-bimodule isomorphism ${ }_{\chi} \mathcal{E}_{\psi}=\varpi^{*}\left({ }_{\chi} \mathcal{A}_{\psi}\right)$.

(ii) Assume that $R^{q} f_{*} \mathcal{O}_{\mathcal{M N}}(\chi-\psi)=0$ holds for all $q>0$. Then, there is a graded $\left(\operatorname{grA}_{\chi}\right)$-(gr $\left.\mathrm{A}_{\psi}\right)$-bimodule isomorphism $\operatorname{gr}^{\mathcal{R}} \Gamma\left(\mathcal{M}^{(1)},{ }_{\chi} \mathcal{A}_{\psi}\right) \cong \Gamma\left(\mathcal{M}, \mathcal{O}_{\mathcal{M}}(\chi-\psi)\right)$. Moreover, we have

$$
R^{q} f_{*}^{(1)}\left({ }_{\chi} \mathcal{A}_{\psi}\right)=0 \quad \text { and } \quad H^{q}\left(\mathcal{M}^{(1)},{ }_{\chi} \mathcal{A}_{\psi}\right)=0, \quad \forall q>0 .
$$

Proof. We argue as in Proposition 4.3.3, and show first that

$$
\left.\left(\left(\operatorname{Id}_{\mathbb{A}^{1}} \times \varpi\right) . \mathcal{R}\left({ }_{\chi} \mathcal{E}_{\psi}\right)\right)^{\psi-\chi}\right|_{\{0\} \times \mathcal{M}(1)} \cong \operatorname{Fr} \cdot \mathcal{O}_{\mathcal{M}}(\psi-\chi) .
$$

Now, the sheaf $\operatorname{Fr} \cdot \mathcal{O}_{\mathcal{M}}(\psi-\chi)$ is clearly locally free. Hence, arguing as at the beginning of subsection 4.5 below we deduce that the sheaf $\left.\mathcal{R}\left({ }_{\chi} \mathcal{E}_{\psi}\right)\right|_{\{0\} \times M^{(1)}}$ is a locally free sheaf on $\mathbf{M}^{(1)}$ which is isomorphic to $\varpi^{*} \operatorname{Fr} . \mathcal{O}_{\mathcal{M}}(\psi-\chi)$. Using Lemma 4.2 .5 one shows that $\mathcal{R}\left({ }_{\chi} \mathcal{E}_{\psi}\right)$ is a locally free sheaf on $\mathbb{A}^{1} \times \mathrm{M}^{(1)}$. Thus, $\chi_{\chi} \mathcal{E}_{\psi}=$ $\left.\mathcal{R}\left({ }_{\chi} \varepsilon_{\psi}\right)\right|_{\{1\} \times \mathrm{M}^{(1)}}$ is a locally free sheaf on $\mathrm{M}^{(1)}$ and, moreover, we have ${ }_{\chi} \mathcal{E}_{\psi}=$ $\varpi^{*}\left({ }_{\chi} \mathcal{A}_{\psi}\right)$.

Remark 4.4.3. The above argument shows that there is a flat family (over $\mathbb{A}^{1}$ ) of coherent sheaves such that nonzero members of the family are all isomorphic to ${ }_{\chi} \mathcal{A}_{\psi}$ and the fiber over $0 \in \mathbb{A}^{1}$ is isomorphic to $\operatorname{Fr} . \mathcal{O}_{\mathcal{M}}(\psi-\chi)$. In particular, in the Grothendieck group of coherent sheaves on $\mathcal{N}^{(1)}$ one has an equality $\left[{ }_{\chi} \mathcal{A}_{\psi}\right]=$ $\left[\operatorname{Fr}_{.} \mathcal{O}_{\mathcal{M}}(\psi-\chi)\right]$. 
4.5. Proof of Theorem 4.1.4. We have $\mathcal{A}_{\chi}=\varpi_{\bullet}\left(\left.\varepsilon_{\chi}\right|_{\mathrm{M}^{(1)}}\right)^{A^{(1)}}$. The sheaf $\mathcal{A}_{\chi}$ in the left-hand side of this equality is locally free, by Proposition 4.3.3 (i). It follows that the sheaf $\left.\mathcal{E}_{\chi}\right|_{M^{(1)}}$ is also locally free, moreover, we have $\left.\mathcal{E}_{\chi}\right|_{M^{(1)}} \cong \varpi^{*} \mathcal{A}_{\chi}$; see (4.1.6).

Now, let $x \in \mathrm{M}^{(1)}$ and write $\bar{x}:=\varpi(x) \in \mathcal{M}^{(1)}$ for its image. Let $\mathcal{E}_{x}$, resp. $\mathcal{A}_{\bar{x}}$, denote the geometric fiber at $x$, resp. at $\bar{x}$, of the corresponding locally free sheaf. We deduce from $\left.\mathcal{E}_{\chi}\right|_{M^{(1)}} \cong \varpi^{*} \mathcal{A}_{\chi}$ that there is an algebra isomorphism

$$
\mathcal{E}_{x} \cong \mathcal{A}_{\bar{x}}, \quad \text { for all } \quad x \in \mathrm{M}^{(1)} \text { and } \quad \bar{x}:=\varpi(x) \in \mathcal{M}^{(1)} \text {. }
$$

Thus, to prove that $\mathcal{A}_{\chi}$ is an Azumaya algebra, it suffices to show that $\mathcal{E}_{x}$ is a matrix algebra, for any $x \in \mathrm{M}^{(1)}$. By definition, we have $\mathcal{E}_{x}=\left(\mathcal{D}_{x}^{(1)} / \mathcal{D}_{x}^{(1)} \cdot \mathfrak{i}_{\chi}\right)^{A_{1}}$, where $\mathcal{D}_{x}^{(1)}$ is the geometric fiber at $x$ of the sheaf $\mathcal{D}^{(1)}$.

We know that $\mathcal{D}^{(1)}$ is an Azumaya algebra on $T^{*} X^{(1)}$. Hence, there is a vector space $E$ and an algebra isomorphism $\mathcal{D}_{x}^{(1)} \cong \operatorname{End}_{\mathbb{k}} E$. Using this, from the last statement of Lemma 3.4.4 we deduce the following algebra isomorphisms:

$$
\mathcal{E}_{x}=\left(\mathcal{D}_{x}^{(1)} / \mathcal{D}_{x}^{(1)} \cdot \mathfrak{i}_{\chi}\right)^{A_{1}} \cong\left(\operatorname{End} E / \text { End } E \cdot \mathfrak{i}_{\chi}\right)^{A_{1}}=\mathrm{D}_{\chi} \cong \operatorname{End}_{\mathbb{k}}\left(E_{\chi}\right) .
$$

Thus, $\mathcal{E}_{x}$ is a matrix algebra, as claimed. This proves that $\mathcal{A}_{\chi}$ is an Azumaya algebra.

To complete the proof of part (i) of the theorem, consider the following chain of canonical algebra maps

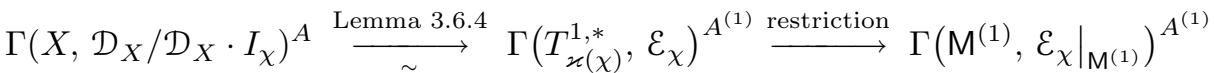

$$
\begin{aligned}
& \cong \Gamma\left(\mathcal{M}^{(1)}, \varpi_{\cdot}\left(\left.\mathcal{E}_{\chi}\right|_{\mathrm{M}^{(1)}}\right)\right)^{A^{(1)}} \cong \Gamma\left(\mathcal{M}^{(1)}, \varpi_{\cdot}\left(\left.\mathcal{E}_{\chi}\right|_{\mathrm{M}^{(1)}}\right)^{A^{(1)}}\right) \cong \Gamma\left(\mathcal{M}^{(1)}, \mathcal{A}_{\chi}\right)
\end{aligned}
$$

The composite map provides the algebra map claimed in part (i) of Theorem 4.1.4.

Part (ii) of the theorem follows directly from the cohomology vanishing in Proposition 4.3.3(i); To prove (iv), we fix a point $x \in \mathrm{M}^{(1)}$, and write $\mathcal{D}_{x}^{(1)} \cong \operatorname{End}_{\mathbb{k}} E$. We know that for the geometric fibers (at $x$ ) of the Azumaya algebras $\varepsilon_{\chi}$, resp., $\mathcal{E}_{\psi}$, one has the following formulas:

$$
\left(\mathcal{E}_{\chi}\right)_{x}=\operatorname{End}_{\mathbb{k}} E_{\chi}, \quad \operatorname{resp} . \quad\left(\mathcal{E}_{\psi}\right)_{x}=\operatorname{End}_{\mathbb{k}} E_{\psi} .
$$

Further, the sheaf $\mathcal{E}_{\psi}$ is locally free by Lemma 4.4.1(i). The fiber of that sheaf at $x$ is an $\left(\mathcal{E}_{\chi}\right)_{x^{-}}\left(\mathcal{E}_{\psi}\right)_{x^{-b i m o d u l e}}$, and Lemma 3.4.4(ii) yields the following $\left(\mathcal{E}_{\chi}\right)_{x^{-}}\left(\mathcal{E}_{\psi}\right)_{x^{-}}$ bimodule isomorphisms; cf. (3.4.3):

$$
\begin{aligned}
\left({ }_{\chi} \mathcal{E}_{\psi}\right)_{x} & \cong \operatorname{Hom}_{\mathcal{D}_{x}^{(1)}}\left(\mathcal{D}_{x}^{(1)} / \mathcal{D}_{x}^{(1)} \cdot \mathfrak{i}_{\chi}, \mathcal{D}_{x}^{(1)} / \mathcal{D}_{x}^{(1)} \cdot \mathfrak{i}_{\psi}\right)^{\psi-\chi} \\
& \cong \operatorname{Hom}_{\text {End } E}\left(\text { End } E / \text { End } E \cdot \mathfrak{i}_{\chi}, \text { End } E / \text { End } E \cdot \mathfrak{i}_{\psi}\right)^{\psi-\chi} \\
& ={ }_{\psi} \mathrm{D}_{\chi} \cong \operatorname{Hom}_{\mathbb{k}}\left(E_{\chi}, E_{\psi}\right) .
\end{aligned}
$$

Thus, the sheaf $\mathcal{E}_{\psi}$ is a sheaf of locally-projective $\mathcal{E}_{\chi}-\mathcal{E}_{\psi}$-bimodules. In particular, the Azumaya algebras $\mathcal{E}_{\chi}$ and $\mathcal{E}_{\psi}$ are Morita equivalent.

Now, for $\bar{x}:=\varpi(x) \in \mathcal{M}^{(1)}$, we have $\left({ }_{\chi} \mathcal{A}_{\psi}\right)_{\bar{x}}=\left({ }_{\chi} \mathcal{E}_{\psi}\right)_{x}$. Therefore, we see from (4.5.1) and Lemma 4.4.1)(i) that the sheaf $\chi \mathcal{A}_{\psi}$ is a sheaf of locally-projective $\mathcal{A}_{\chi}-\mathcal{A}_{\psi}$-bimodules. Hence, it provides the required Morita equivalence between the Azumaya algebras $\mathcal{A}_{\chi}$ and $\mathcal{A}_{\psi}$. 
Part (iii) of Theorem 4.1.4 is a special case of the following more general result due to [BK, Proposition 2.2].

Proposition 4.5.3. Let $\mathcal{M}$ be a smooth connected variety over $\mathbb{k}$ with the trivial canonical class, and such that the morphism $\mathcal{M} \rightarrow \mathcal{M}_{\mathrm{aff}}$ is proper. Let $\mathcal{A}$ be an Azumaya algebra on $\mathcal{M}$ such that $H^{i}(\mathcal{M}, \mathcal{A})=0, \forall i>0$ and, moreover, the algebra $\mathrm{A}:=\Gamma(\mathcal{M}, \mathcal{A})$ has finite homological dimension.

Then, the functor below provides an equivalence beteen the bounded derived categories of sheaves of coherent $\mathcal{A}$-modules and finitely-generated $\mathrm{A}$-modules, respectively:

$$
D^{b}(\mathcal{A} \text {-Mod }) \longrightarrow D^{b}\left(\mathrm{~A}-\operatorname{Mod}^{f i n . ~ g e n .}\right), \quad \mathcal{F} \longmapsto \operatorname{RHom}_{\mathcal{A}-\operatorname{Mod}}(\mathcal{A}, \mathcal{F}) .
$$

The proof of this proposition exploits the technique of Serre functors, and is similar in spirit to the proof of [BKR, Theorem 2.4].

Part (iii) of our theorem follows from the proposition since for any $\mathcal{A}$-module $\mathcal{F}$, one has $\operatorname{RHom}_{\mathcal{A}-\operatorname{Mod}}(\mathcal{A}, \mathcal{F}) \cong \operatorname{RHom}_{\mathcal{O}_{\mathcal{M}}-\operatorname{Mod}}\left(\mathcal{O}_{\mathcal{M}}, \mathcal{F}\right)=\operatorname{R} \Gamma(\mathcal{M}, \mathcal{F})$.

This completes the proof of the Theorem 4.1.4.

\section{The Rational Cherednik algebra of type $\mathbf{A}_{n-1}$.}

5.1. Basic definitions. Let $W:=S_{n}$ denote the Symmetric group and let $\mathbb{Z}[W]$ denote the group algebra of $W$. Write $s_{i j} \in W$ for the transposition $i \leftrightarrow j$. We consider two sets of variables $x_{1}, \ldots, x_{n}$, and $y_{1}, \ldots, y_{n}$, and let $W=S_{n}$ act on the polynomial algebras $\mathbb{Z}\left[x_{1}, \ldots, x_{n}\right]$ and $\mathbb{Z}\left[y_{1}, \ldots, y_{n}\right]$ by permutation of the variables.

Let $\mathbf{c}$ be an indeterminate. We define the rational Cherednik algebra of type $\mathbf{A}_{n-1}$ as a $\mathbb{Z}[\mathbf{c}]$-algebra, $\mathrm{H}$, with generators $x_{1}, \ldots, x_{n}, y_{1}, \ldots, y_{n}$ and $\mathbb{Z}[W]$, and the following defining relations; see [EG]:

$$
\begin{array}{rlrl}
s_{i j} \cdot x_{i} & =x_{j} \cdot s_{i j}, & s_{i j} \cdot y_{i}=y_{j} \cdot s_{i j}, & \forall i, j \in\{1,2, \ldots, n\}, i \neq j, \\
{\left[y_{i}, x_{j}\right]} & =\mathbf{c} \cdot s_{i j}, \quad\left[x_{i}, x_{j}\right]=0=\left[y_{i}, y_{j}\right], & \forall i, j \in\{1,2, \ldots, n\}, i \neq j, \\
{\left[y_{k}, x_{k}\right]=1-\mathbf{c} \cdot \sum_{i \neq k} s_{i k} .} &
\end{array}
$$

Given a field $\mathbb{k}$ and $c \in \mathbb{k}$, we let $\mathrm{H}_{c}:=\mathbb{k} \otimes_{\mathbb{Z}[\mathbf{c}]} \mathrm{H}$ be the $\mathbb{k}$-algebra obtained from $\mathrm{H}$ by extension of scalars via the homomorphism $\mathbb{Z}[\mathbf{c}] \rightarrow \mathbb{k}, f \longmapsto f(c)$.

We keep our standing assumption char $\mathbb{k}>n$, and write $\mathrm{e}=\frac{1}{n !} \sum_{g \in W} g \in$ $\mathbb{k}[W] \subset \mathrm{H}_{c}$ for the symmetrizer idempotent. Let $\mathrm{eH}_{c} \mathrm{e} \subset \mathrm{H}_{c}$ be the spherical subalgebra; see EG.

Let $\mathfrak{h}:=\mathbb{k}^{n}$ be the tautological permutation representation of $W$. We identify the variables $x_{1}, \ldots, x_{n}$, resp. $y_{1}, \ldots, y_{n}$, with coordinates on $\mathfrak{h}$, resp. on $\mathfrak{h}^{*}$. The algebras $\mathrm{H}_{c}$ and $\mathrm{eH}_{c}$ e come equipped with compatible increasing filtrations such that all elements of $W$ and $x_{i} \in \mathfrak{h}^{*} \subset \mathrm{H}_{c}$ have filtration degree zero, and elements $y_{i} \in \mathfrak{h} \subset \mathrm{H}_{c}$ have filtration degree 1 .

The Poincaré-Birkhoff-Witt theorem for rational Cherednik algebras (cf. [EG]) yields graded algebra isomorphisms

$$
\operatorname{gr} \mathrm{H}_{c} \cong \mathbb{k}\left[\mathfrak{h}^{*} \times \mathfrak{h}\right] \# W, \text { and } \operatorname{gr}\left(\mathrm{eH}_{c} \mathrm{e}\right) \cong \mathbb{k}\left[\mathfrak{h}^{*} \times \mathfrak{h}\right]^{W} .
$$

5.2. Dunkl representation. Write $\mathfrak{h}^{\text {reg }}$ for an affine Zariski open dense subset of $\mathfrak{h}$ formed by points with pairwise distinct coordinates. The group $W$ acts naturally on the algebra $\mathcal{D}\left(\mathfrak{h}^{\text {reg }}\right)$ of crystalline differential operators on $\mathfrak{h}^{\text {reg }}$ and 
we let $\mathcal{D}\left(\mathfrak{h}^{\text {reg }}\right)^{W} \subset \mathcal{D}\left(\mathfrak{h}^{\text {reg }}\right)$ be the subalgebra of $W$-invariant differential operators. The standard increasing filtration on the algebra of differential operators induces an increasing filtration on the subalgebra $\mathcal{D}\left(\mathfrak{h}^{\text {reg }}\right)^{W}$, and we have $\operatorname{gr} \mathcal{D}\left(\mathfrak{h}^{\text {reg }}\right)^{W} \cong \mathbb{k}\left[T^{*} \mathfrak{h}^{\text {reg }}\right]^{W}=\mathbb{k}\left[\mathfrak{h}^{*} \times \mathfrak{h}^{\text {reg }}\right]^{W}$.

According to Cherednik (see also [EG], DO]), there is an injective algebra homomorphism

$$
\Theta_{c}: \mathrm{eH}_{c} \mathrm{e} \hookrightarrow \mathcal{D}\left(\mathfrak{h}^{\mathrm{reg}}\right)^{W},
$$

called Dunkl representation of the algebra $\mathrm{eH}_{c} \mathrm{e}$.

Let $\mathrm{B}_{c}:=\Theta_{c}\left(\mathrm{eH}_{c} \mathrm{e}\right) \subset \mathcal{D}\left(\mathfrak{h}^{\text {reg }}\right)^{W}$ be the image of the Dunkl representation. We equip the algebra $B_{c}$ with increasing filtration induced from the standard one on $\mathcal{D}\left(\mathfrak{h}^{\text {reg }}\right)^{W}$. Then, gr $\boldsymbol{B}_{c}$ becomes a graded subalgebra in gr $\mathcal{D}\left(\mathfrak{h}^{\text {reg }}\right)^{W}=\mathbb{k}\left[\mathfrak{h}^{*} \times \mathfrak{h}^{\text {reg }}\right]^{W}$, which is known to be equal to $\mathbb{k}\left[\mathfrak{h}^{*} \times \mathfrak{h}\right]^{W} \subset \mathbb{k}\left[\mathfrak{h}^{*} \times \mathfrak{h}^{\text {reg }}\right]^{W}$; see [EG. Further, the map $\Theta_{c}$ in (5.2.1) is known to be filtration preserving, and it was proved in EG] that the associated graded map, gr $\Theta_{c}$, induces graded algebra isomorphisms

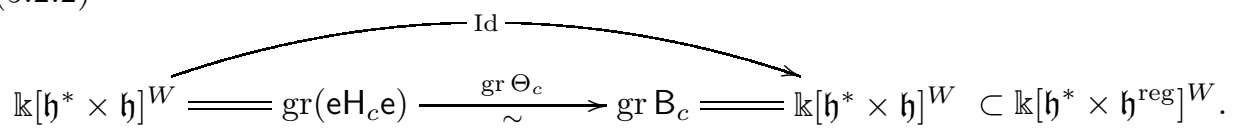

5.3. The "radial part" construction. In this section, we let $\mathbb{k}$ be an arbitrary algebraically closed field, either of characteristic zero or of characteristic $p$.

Let $V$ be an $n$-dimensional vector space over $\mathbb{k}$. In case the field $\mathbb{k}$ has finite characteristic we assume throughout that char $\mathbb{k}>n \geq 2$.

We put $G=\operatorname{GL}(V)$ and let $\mathfrak{g}=\operatorname{Lie} G=\mathfrak{g l}(V)$ be the Lie algebra of $G$. We consider the vector space $\mathfrak{G}:=\mathfrak{g} \times V$.

Definition 5.3.1. Let $\mathfrak{G}^{\circ} \subset \mathfrak{G}=\mathfrak{g} \times V$ be a Zariski open dense subset formed by the pairs $(x, v)$ such that $v$ is a cyclic vector for the operator $x: V \rightarrow V$.

We recall that the endomorphism $x \in \mathfrak{g}$ admits a cyclic vector if and only if $x$ is regular (not necessarily semisimple), i.e., the centralizer of $x$ in $\mathfrak{g}$ has dimension $n$.

Fix a nonzero volume element vol $\in \wedge^{n} V^{*}$. We introduce the following polynomial function on $\mathfrak{G}$ :

$$
(x, v) \longmapsto \mathbf{s}(x, v):=\left\langle\operatorname{vol}, v \wedge x(v) \wedge \ldots \wedge x^{n-1}(v)\right\rangle .
$$

It is clear that we have $\mathfrak{G}^{\circ}=\mathfrak{G} \backslash \mathbf{s}^{-1}(0)$, in particular, $\mathfrak{G}^{\circ}$ is an affine variety.

The group $G$ acts on $\mathfrak{g}$ via the adjoint action, and acts naturally on $V$. This gives a $G$-diagonal action on $\mathfrak{G}$ such that $\mathfrak{G}^{\circ}$ is a $G$-stable subset of $\mathfrak{G}$. We compose the first projection $\mathfrak{G}=\mathfrak{g} \times V \rightarrow \mathfrak{g}$ with the adjoint quotient map $\mathfrak{g} \rightarrow \mathfrak{g} / \operatorname{Ad} G=\mathfrak{h} / W$, and restrict the resulting morphism to the subset $\mathfrak{G}^{\circ} \subset \mathfrak{G}$. This way we get a morphism $p: \mathfrak{G}^{\circ} \rightarrow \mathfrak{h} / W$. The group $G$ clearly acts along the fibers of $p$, and we have the following well-known result.

Lemma 5.3.3. (i) The $G$-action on $\mathfrak{G}^{\circ}$ is free and each fiber of $p$ is a single $G$-orbit;

(ii) Furthermore, the map $p: \mathfrak{G}^{\circ} \rightarrow \mathfrak{h} / W$ is a universal geometric quotient morphism.

It follows from the lemma that $\mathfrak{G}^{\circ}$ is a principal $G$-bundle over $\mathfrak{h} / W$.

Given an integer $c \in \mathbb{Z}$, we put

$$
\mathcal{O}\left(\mathfrak{G}^{\circ}, c\right):=\left\{f \in \mathbb{k}\left[\mathfrak{G}^{\circ}\right] \mid g^{*}(f)=(\operatorname{det} g)^{c} \cdot f, \quad \forall g \in G\right\} .
$$


It is clear that pull-back via the bundle projection $p$ makes $\mathcal{O}\left(\mathfrak{G}^{\circ}, c\right)$ a $\mathbb{k}[\mathfrak{h} / W]$ module. Also, observe that $\mathbf{s} \in \mathcal{O}\left(\mathfrak{G}^{\circ}, 1\right)$.

Corollary 5.3.4. For any $c \in \mathbb{Z}$, the space $\mathcal{O}\left(\mathfrak{G}^{\circ}, c\right)$ is a rank one free $\mathbb{k}[\mathfrak{h} / W]$ module with generator $\mathbf{s}^{c}$.

Notation 5.3.5. For $c \in \mathbb{k}$, we consider a Lie algebra homomorphism $\chi_{c}: \mathfrak{g} \rightarrow$ $\mathbb{k}, x \longmapsto c \cdot \operatorname{tr}(x)$. Let $I_{c}:=I_{\chi_{c}} \subset \mathcal{U} \mathfrak{g}$, denote the two-sided ideal generated by the elements $\left\{x-\chi_{c}(x)\right\}_{x \in \mathfrak{g}}$; cf. Definition 3.3.1.

The action of $G$ on $\mathfrak{G}^{\circ}$ induces an algebra map $\mathcal{U} \mathfrak{g} \rightarrow \mathcal{D}\left(\mathfrak{G}^{\circ}\right)$. We fix $c \in \mathbb{k}$, and perform the Hamiltonian reduction of the sheaf $\mathcal{D}_{\mathfrak{G}^{\circ}}$, of crystalline differential operators on $\mathfrak{G}^{\circ}$, at the point $\chi_{c}$. This way, we get an associative algebra $\left[\mathcal{D}\left(\mathfrak{G}^{\circ}\right) / \mathcal{D}\left(\mathfrak{G}^{\circ}\right) \cdot I_{C}\right]^{G}$.

From Lemma 5.3 .3 and the isomorphism on the right of formula (3.7.2) we deduce the following.

Proposition 5.3.6. For any $c \in \mathbb{k}$, there is a natural algebra isomorphism

$$
\left[\mathcal{D}\left(\mathfrak{G}^{\circ}\right) / \mathcal{D}\left(\mathfrak{G}^{\circ}\right) \cdot I_{c}\right]^{G} \cong \mathcal{D}(\mathfrak{h} / W) .
$$

More explicitly, if $c$ is an integer, then the isomorphism of the proposition is obtained by transporting the action of differential operators on $\mathfrak{G}^{\circ}$ via the bijection $\mathbb{k}[\mathfrak{h} / W] \stackrel{\sim}{\longrightarrow} \mathcal{O}\left(\mathfrak{G}^{\circ}, c\right), f \longmapsto \mathbf{s}^{c} \cdot p^{*}(f)$, provided by Corollary 5.3.4.

Remark 5.3.7. The explicit construction of the isomorphism shows, in particular, that the algebra $\mathcal{D}\left(\mathfrak{h} / W, \chi_{c}\right)$ of twisted differential operators coming from the righthand side of the general formula (3.7.2) turns out to be canonically isomorphic, in our case, to the algebra $\mathcal{D}(\mathfrak{h} / W)$ of ordinary differential operators. Thus, we have put $\mathcal{D}(\mathfrak{h} / W)$ on the right-hand side of the isomorphism of Proposition 5.3.6 (although we have only justified this for integral values of $c$, the same holds for arbitrary values of $c$ as well).

Observe further that the algebras $\left[\mathcal{D}\left(\mathfrak{G}^{\circ}\right) / \mathcal{D}\left(\mathfrak{G}^{\circ}\right) \cdot I_{c}\right]^{G}$ and $\mathcal{D}(\mathfrak{h} / W)$ both come equipped with natural increasing filtrations and the isomorphism of the proposition is filtration preserving.

The isomorphism of Proposition [5.3.6 may be viewed as a refined version of the "radial part" construction considered in EG.

Remark 5.3.8. The action of differential operators on $\mathbb{k}\left[\mathfrak{h}^{\text {reg }}\right]^{W}$ gives rise to the following natural algebra inclusions: $\mathcal{D}(\mathfrak{h})^{W} \subset \mathcal{D}(\mathfrak{h} / W) \subset \mathcal{D}\left(\mathfrak{h}^{\text {reg }}\right)^{W}$. We also remark that the space $\mathcal{D}(\mathfrak{h})^{W}$ has infinite codimension in $\mathcal{D}(\mathfrak{h} / W)$.

5.4. A Harish-Chandra homomorphism. Let $\mathfrak{g}^{\text {rs }} \subset \mathfrak{g}$ denote the Zariski open dense subset of semisimple regular elements. Observe that the eigenspaces of an element $x \in \mathfrak{g}^{\mathrm{rs}}$ give a direct sum decomposition $V=\ell_{1} \oplus \ldots \oplus \ell_{n}$. Hence, any $v \in V$ we can be uniquely written as $v=v_{1}+\ldots+v_{n}$ where $v_{i} \in \ell_{i}, i=1, \ldots, n$. Such a vector $v$ is a cyclic vector for $x$ if and only if none of the $v_{i}$ 's vanish.

We put $U:=\left\{(x, v) \in \mathfrak{G}^{\circ} \mid x \in \mathfrak{g}^{\text {rs }}\right\}$. Thus, $U$ is an affine $G$-stable Zariski open dense subset in $\mathfrak{G}^{\circ}$, and the geometric quotient morphism $p: \mathfrak{G}^{\circ} \rightarrow \mathfrak{h} / W$ restricts to a geometric quotient morphism $p: U \rightarrow \mathfrak{h}^{\text {reg }} / W$.

Now, the group $G$ acts naturally on the algebra $\mathbb{k}[\mathfrak{G}]$. We observe that the first projection $\mathfrak{G}=\mathfrak{g} \times V \rightarrow \mathfrak{g}$ induces an isomorphism of $G$-invariants $\mathbb{k}[\mathfrak{g}]^{G} \stackrel{\sim}{\longrightarrow}$ 
$\mathbb{k}[\mathfrak{G}]^{G}$, since the center of $G$ acts trivially on $\mathbb{k}[\mathfrak{g}]$ and nontrivially on any homogeneous polynomial $f \in \mathbb{k}[V]$ such that $\operatorname{deg} f>0$.

Let $\Delta_{\mathfrak{g}}$ denote the second order Laplacian on $\mathfrak{g}$ associated to a nondegenerate invariant bilinear form. We will identify $\Delta_{\mathfrak{g}}$ with the operator $\Delta_{\mathfrak{g}} \otimes 1 \in \mathcal{D}(\mathfrak{g}) \otimes \mathcal{D}(V)$ $=\mathcal{D}(\mathfrak{G})$ acting trivially in the $V$-direction. Restricting the latter differential operator to $U$, we may view $\Delta_{\mathfrak{g}}$ as an element of the algebra $\Gamma\left(U, \mathcal{D}_{U} / \mathcal{D}_{U} \cdot I_{C}\right)^{G}$.

Write $x_{1}, \ldots, x_{n}$ for coordinates in $\mathfrak{h}=\mathbb{k}^{n}$.

Proposition 5.4.1. For any $c \in \mathbb{k}$, there is a natural filtration preserving algebra isomorphism $\Psi_{c}: \Gamma\left(U, \mathcal{D}_{U} / \mathcal{D}_{U} I_{c}\right)^{G} \stackrel{\sim}{\longrightarrow} \mathcal{D}\left(\mathfrak{h}^{\text {reg }}\right)^{W}$, that reduces to the "Chevalley restriction" map,

$$
\left.f \longmapsto f\right|_{\mathfrak{h} \times\{0\}}, \quad \mathbb{k}[\mathfrak{g} \times V]^{G} \longrightarrow \mathbb{k}\left[\mathfrak{h}^{\text {reg }} \times\{0\}\right]^{W}=\mathbb{k}\left[\mathfrak{h}^{\text {reg }}\right]^{W},
$$

on polynomia国 zero order differential operators, and such that $\Psi_{c}\left(\Delta_{\mathfrak{g}}\right)=\mathrm{L}_{c}$, where

$$
\mathrm{L}_{c}=\sum_{j} \frac{\partial^{2}}{\partial x_{j}^{2}}-\sum_{i \neq j} \frac{c(c+1)}{\left(x_{i}-x_{j}\right)^{2}}
$$

is the Calogero-Moser operator with rational potential, corresponding to the parameter $c$.

Sketch of Proof. We restrict the isomorphism of Proposition 5.3.6 to $U \subset \mathfrak{G}^{\circ}$, equivalently, we apply formula (3.7.2) to the geometric quotient morphism $p: U \rightarrow$ $\mathfrak{h}^{\text {reg }} / W$ and to the character $\chi_{c}: \mathfrak{g} \rightarrow \mathbb{k}$. This way, we deduce an algebra isomorphism

$$
\Gamma\left(U, \mathcal{D}_{U} / \mathcal{D}_{U} \cdot I_{c}\right)^{G} \stackrel{\sim}{\longrightarrow} \mathcal{D}\left(\mathfrak{h}^{\text {reg }} / W\right)
$$

Now, the natural projection $\mathfrak{h}^{\text {reg }} \rightarrow \mathfrak{h}^{\text {reg }} / W$ is a Galois covering with Galois group $W$. Therefore, pull-back via the projection gives rise to a canonical isomorphism $\mathcal{D}\left(\mathfrak{h}^{\text {reg }} / W\right) \cong \mathcal{D}\left(\mathfrak{h}^{\text {reg }}\right)^{W}$. Thus, composing with (5.4.3) yields an algebra isomorphism $\Psi_{c}^{\prime}: \Gamma\left(U, \mathcal{D}_{U} / \mathcal{D}_{U} \cdot I_{c}\right)^{G} \stackrel{\sim}{\longrightarrow} \mathcal{D}\left(\mathfrak{h}^{\text {reg }}\right)^{W}$.

Finally, let $R_{+}$be the set of positive roots of our root system $R \subset \mathfrak{h}^{*}$ of type $\mathbf{A}_{n-1}$, and set $\delta:=\prod_{\alpha \in R_{+}} \alpha$. We conjugate the map $\Psi_{c}^{\prime}$ by $\delta$. That is, for any $u \in \Gamma\left(U, \mathcal{D}_{U} / \mathcal{D}_{U} \cdot I_{c}\right)^{G}$, let $\Psi_{c}(u)$ be a differential operator on $\mathfrak{h}^{\text {reg }}$ given by $\Psi_{c}(u):=M_{\delta} \circ \Psi_{c}^{\prime}(u) \circ M_{1 / \delta}$, where $M_{f}$ denotes the operator of multiplication by a function $f \in \mathbb{k}\left[\mathfrak{h}^{\mathrm{reg}}\right]$. The map $u \mapsto \Psi_{c}(u)$ thus defined gives the isomorphism $\Psi_{c}$ of the proposition.

The equation $\Psi_{c}\left(\Delta_{\mathfrak{g}}\right)=\mathrm{L}_{c}$ is verified by a direct computation similar to one in the proof of [EG, Proposition 6.2]. We leave the details to the reader.

We will need the following analogue of the surjectivity part of [EG, Corollary 7.4].

Proposition 5.4.4. Let $\mathbb{k}=\mathbb{Q}$ be the field of rational numbers. Then, for all $c \in \mathbb{k}$, the algebra $\mathrm{B}_{c}$ is contained in the image of the following composite map; cf. Proposition 5.4.1:

$\bar{\Psi}_{c}: \quad \Gamma\left(\mathfrak{G}, \mathcal{D}_{\mathfrak{G}} / \mathcal{D}_{\mathfrak{G}} \cdot I_{c}\right)^{G} \underset{\text { restriction }}{\longrightarrow} \Gamma\left(U, \mathcal{D}_{U} / \mathcal{D}_{U} \cdot I_{c}\right)^{G} \stackrel{\Psi_{c}}{\sim} \mathcal{D}\left(\mathfrak{h}^{\mathrm{reg}}\right)^{W}$.

\footnotetext{
${ }^{1}$ Notice that although $\mathfrak{h}^{\text {reg }} \times\{0\}$ is not a subset of $U$ the restriction to $\mathfrak{h}^{\text {reg }} \times\{0\}$ is well defined for a polynomial on $\mathfrak{G}=\mathfrak{g} \times V$.
} 
Proof. We repeat the argument used in [EG], which is quite standard.

Specifically, the algebra $\mathrm{B}_{c}$ contains a subalgebra $\mathrm{C}_{c} \subset \mathrm{B}_{c}$ formed by so-called Calogero-Moser integrals. The algebra $\mathrm{C}_{c}$ is a commutative algebra containing the Calogero-Moser operator $\mathrm{L}_{c}$, and isomorphic to $(\operatorname{Sym} \mathfrak{h})^{W}$, due to a result by Opdam. Moreover, the associated graded map corresponding to the imbedding $\mathrm{C}_{c} \hookrightarrow \mathrm{B}_{c}$ induces an isomorphism; cf. (5.2.2):

$$
\operatorname{gr} C_{c} \cong(\operatorname{Sym} \mathfrak{h})^{W} \hookrightarrow\left(\operatorname{Sym}\left(\mathfrak{h} \oplus \mathfrak{h}^{*}\right)\right)^{W}=\mathbb{k}\left[\mathfrak{h}^{*} \times \mathfrak{h}\right]^{W} .
$$

Observe next that the imbedding $\mathfrak{g}=\mathfrak{g} \times\{0\} \hookrightarrow \mathfrak{g} \times V=\mathfrak{G}$ induces an isomorphism $(\operatorname{Sym} \mathfrak{g})^{G} \stackrel{\sim}{\longrightarrow}(\operatorname{Sym} \mathfrak{G})^{G}$, very similar to the isomorphism $\mathbb{k}[\mathfrak{g}]^{G} \stackrel{\sim}{\longrightarrow}$ $\mathbb{k}[\mathfrak{G}]^{G}$ explained in subsection $\left[5.3\right.$. Thus, we identify $(\operatorname{Sym} \mathfrak{g})^{G}$ with $(\operatorname{Sym} \mathfrak{G})^{G}$, and view the latter as a subalgebra in $\mathcal{D}(\mathfrak{G})^{G}$ formed by constant coefficient differential operators. Clearly, this is a commutative subalgebra that contains $\Delta_{\mathfrak{g}}=\Delta_{\mathfrak{g}} \otimes 1 \in$ $\mathcal{D}(\mathfrak{G})^{G}$.

The homomorphism $\Psi_{c}$ of Proposition [5.4.1, hence the composite map $\bar{\Psi}_{c}$ of Proposition 5.4.4 takes the algebra $\left(\mathrm{Symg}^{G}\right)^{G}$, viewed as a subalgebra in $\Gamma\left(\mathfrak{G}, \mathcal{D}_{\mathfrak{G}} / \mathcal{D}_{\mathfrak{G}} \cdot I_{c}\right)^{G}$, to a commutative subalgebra of $\mathcal{D}\left(\mathfrak{h}^{\text {reg }}\right)^{W}$ containing $\mathrm{L}_{c}$. Furthermore, one proves by a standard argument that $\bar{\Psi}_{c}\left((\mathrm{Sym} \mathfrak{g})^{G}\right) \subset \mathrm{C}_{c}$ (cf. e.g. [BEG] $) ;$ moreover, the induced map gr $\bar{\Psi}_{c}: \operatorname{gr}(\operatorname{Sym} \mathfrak{g})^{G} \longrightarrow \operatorname{gr} C_{c}=(\operatorname{Sym} \mathfrak{h})^{W}$ is the Chevalley isomorphism $(\mathrm{Sym} \mathfrak{g})^{G} \stackrel{\sim}{\longrightarrow}(\mathrm{Sym} \mathfrak{h})^{W}$. It follows that the map $\bar{\Psi}_{c}$ induces an isomorphism $(\operatorname{Sym} \mathfrak{g})^{G} \stackrel{\sim}{\longrightarrow} C_{c}$.

Now the algebra $B_{c}$ is known to be generated by the two subalgebras $\mathbb{k}\left[\mathfrak{h}^{*}\right]^{W}$ and $C_{c}$ (since gr $B_{c}=\mathbb{k}\left[\mathfrak{h}^{*} \times \mathfrak{h}\right]^{W}$, viewed as a Poisson algebra with respect to the natural Poisson structure on $\mathfrak{h}^{*} \times \mathfrak{h}=T^{*} \mathfrak{h}$, is known to be generated by the two subalgebras $\mathbb{k}\left[\mathfrak{h}^{*}\right]^{W}$ and $\left.\mathbb{k}[\mathfrak{h}]^{W}\right)$. By Proposition 5.4 .1 , we have $\bar{\Psi}_{c}\left(\mathbb{k}[\mathfrak{G}]^{G}\right)=\mathbb{k}\left[\mathfrak{h}^{*}\right]^{W}$ and as we have explained above, one also has $\bar{\Psi}_{c}\left((\operatorname{Sym} \mathfrak{g})^{G}\right)=\mathrm{C}_{c}$. We conclude that $\mathrm{B}_{c}$ is equal to the subalgebra in $\mathcal{D}\left(\mathfrak{h}^{\text {reg }}\right)^{W}$ generated by $\bar{\Psi}_{c}\left(\mathbb{k}[\mathfrak{G}]^{G}\right)$ and $\mathrm{C}_{c}$, hence, is contained in the image of the map $\bar{\Psi}_{c}$.

\section{An Azumaya algebra on the Hilbert scheme}

6.1. Nakajima construction reviewed. We keep the notations of subsection 5.3 In particular, we have a vector space $V$ over $\mathbb{k}$, such that char $\mathbb{k}>n \geq 2$, where $n=\operatorname{dim} V$. We put $G:=\operatorname{GL}(V)$ and $\mathfrak{g}:=\operatorname{Lie} G=\mathfrak{g l}(V)$. We will freely identify $\mathfrak{g}^{*}$ with $\mathfrak{g}$ via the pairing $\mathfrak{g} \times \mathfrak{g} \rightarrow \mathbb{k},(x, y) \longmapsto \frac{1}{n} \operatorname{tr}(x \cdot y)$.

The group $G$ acts naturally on $V$ and also on $\mathfrak{g}$, via the adjoint action. We consider the $G$-diagonal action on the vector space $\mathfrak{G}=\mathfrak{g} \times V$, and the corresponding Hamiltonian $G$-action on the cotangent bundle: $T^{*} \mathfrak{G}=\mathfrak{G}^{*} \times \mathfrak{G} \cong \mathfrak{g} \times \mathfrak{g} \times V^{*} \times V$. The moment map for this action is given by the formula

(6.1.1) $\mu: T^{*} \mathfrak{G}=\mathfrak{g} \times \mathfrak{g} \times V^{*} \times V \longrightarrow \mathfrak{g}^{*} \cong \mathfrak{g}, \quad(x, y, \check{v}, v) \longmapsto[x, y]+\check{v} \otimes v \in \mathfrak{g}$.

Recall the notation introduced in 5.3.5. Observe that the Lie algebra homomorphism $\chi_{c}=c \cdot \operatorname{tr} \in \mathfrak{g}^{*}$ corresponds, under the identification $\mathfrak{g}^{*} \cong \mathfrak{g}$, to the element $c \cdot \operatorname{Id}_{V} \in \mathfrak{g}$.

Following Nakajima, we introduce the set

$$
\mathfrak{M}_{c}:=\mu^{-1}\left(\chi_{c}\right)=\left\{(x, y, \check{v}, v) \in \mathfrak{g} \times \mathfrak{g} \times V^{*} \times V \mid[x, y]+\check{v} \otimes v=c \cdot \operatorname{Id}_{V}\right\} .
$$

This is an affine algebraic variety equipped with a natural $\mathrm{GL}(V)$-action.

If $c \neq 0$, then $\mathfrak{M}_{c}$ is known (see $[\mathrm{Na1}$, Wi] to be smooth, moreover, the $G$ action on $\mathfrak{M}_{c}$ is free. The quotient $\mathcal{M}_{c}:=\mathfrak{M}_{c} / G$ is a well-defined smooth affine 
algebraic variety of dimension $2 \operatorname{dim} V$, called Calogero-Moser space. It was first considered in [KKS], and studied in Wi]; cf. also [Na1]. By definition, $\mathcal{M}_{c}$ is the Hamiltonian reduction of $T^{*} \mathfrak{G}$ with respect to the 1 -point $G$-orbit $\chi_{c} \in \mathfrak{g}^{*}$. The standard symplectic structure on the cotangent bundle thus induces a symplectic structure on $\mathcal{M}_{c}$.

If $c=0$, then the set $\mathfrak{M}_{0}$ is not smooth, and $G$-action on $\mathfrak{M}_{0}$ is not free. Let $\mathfrak{M}_{0}^{\mathrm{s}}$ be the subset of "stable points" formed by quadruples $(x, y, \check{v}, v) \in \mathfrak{g} \times \mathfrak{g} \times V^{*} \times V$ such that $v \in V$ is a cyclic vector for $(x, y)$, i.e., such that there is no nonzero proper subspace $V^{\prime} \subset V$ that contains $v$ and that is both $x$ - and $y$-stable. Then, $\mathfrak{M}_{0}^{\mathrm{s}}$ is known to be a smooth Zariski open $G$-stable subset in $\mathfrak{M}_{0}$. Moreover, the differential of the moment map $\mu$ (see (6.1.1) is known (cf. [Na1]) to be surjective at any point of $\mathfrak{M}_{0}^{\mathrm{s}}$, and the $G$-action on $\mathfrak{M}_{0}^{\mathrm{s}}$ is free.

The following description of $\mathrm{Hilb}^{n} \mathbb{A}^{2}$, the Hilbert scheme of zero-dimensional length $n$ subschemes in the affine plane $\mathbb{A}^{2}$, is essentially due to Nakajima [Na1].

Proposition 6.1.2. There exists a smooth geometric quotient morphism $\mathfrak{M}_{0}^{\mathrm{s}} \rightarrow$ Hilb $^{n} \mathbb{A}^{2}$.

Remark 6.1.3. It is known that Hilb ${ }^{n} \mathbb{A}^{2}$ is a smooth connected (nonaffine) algebraic variety of dimension $2 \operatorname{dim} V$.

Thus, the Hilbert scheme Hilb ${ }^{n} \mathbb{A}^{2}$ may be viewed as a "Hamiltonian reduction" of $T^{*} \mathfrak{G}$ at the 1 -point $G$-orbit $\{0\} \subset \mathfrak{g}^{*}$. In particular, Hilb ${ }^{n} \mathbb{A}^{2}$ has a natural symplectic structure.

It is known that for any quadruple $(x, y, \check{v}, v) \in \mathfrak{M}_{0}$, the operators $x, y$ can be put simultaneously in the upper-triangular form. Hence, the diagonal components of these two operators give a pair of elements $\operatorname{diag} x, \operatorname{diag} y \in \mathfrak{h}$, well defined up to simultaneous action of $W=S_{n}$. The assignment $(x, y, \check{v}, v) \longmapsto(\operatorname{diag} x, \operatorname{diag} y)$ clearly descends to a morphism $\Upsilon: \operatorname{Hilb}^{n} \mathbb{A}^{2} \longrightarrow(\mathfrak{h} \oplus \mathfrak{h}) / W$, called the HilbertChow morphism. It is known that the Hilbert-Chow morphism induces an algebra isomorphism

$$
\Gamma\left(\operatorname{Hilb}^{n} \mathbb{A}^{2}, \mathcal{O}_{\mathrm{Hilb}_{\mathbb{A}^{2}}}\right) \stackrel{\stackrel{\Upsilon_{*}}{\longrightarrow}}{\longrightarrow} \Gamma\left((\mathfrak{h} \times \mathfrak{h}) / W, \mathcal{O}_{(\mathfrak{h} \times \mathfrak{h}) / W}\right)=\mathbb{k}[\mathfrak{h} \oplus \mathfrak{h}]^{W}
$$

6.2. The Azumaya algebra. Let $\varkappa: \mathbb{A}^{1} \rightarrow \mathbb{A}^{1}, c \mapsto \varkappa(c)=c^{p}-c$ be the classical Artin-Schreier map. This map is related to the map $\varkappa: \mathbb{X}^{*}(\mathfrak{g}) \rightarrow \mathfrak{g}^{1, *}$ defined in (3.2.2) by the formula $\varkappa\left(\chi_{c}\right)=\chi_{\varkappa(c)}=\varkappa(c) \cdot \operatorname{tr}^{(1)}$.

We introduce the following simplified notation for the scheme-theoretic fiber of the moment map $\mu^{(1)}: T^{*} X^{(1)} \rightarrow \mathfrak{g}^{1, *}$ over the point $\varkappa\left(\chi_{c}\right)$ :

$$
T_{\varkappa(c)}^{1, *}:=T_{\varkappa\left(\chi_{c}\right)}^{1, *}=\left[\mu^{(1)}\right]^{-1}\left(\chi_{\varkappa(c)}\right)=\mathfrak{M}_{\varkappa(c)}^{(1)} .
$$

We are going to apply the general Hamiltonian reduction procedure of subsection 4.1 to the algebraic group $A:=G$, the Lie algebra character $\chi=\chi_{\varkappa(c)}$, and the natural $G$-action on the variety $X:=\mathfrak{G}$.

If $\varkappa(c) \neq 0$, then the variety $T_{\varkappa(c)}^{1, *}=\mathfrak{M}_{\varkappa(c)}^{(1)}$ is smooth, the $G$-action on this variety is free, and there is a smooth geometric quotient map $\mathfrak{M}_{\varkappa(c)} \longrightarrow \mathcal{M}_{\varkappa(c)}$, where $\mathcal{M}_{\varkappa(c)}$ is the Calogero-Moser variety with parameter $\varkappa(c)=c^{p}-c$. Thus, the construction of subsection 4.1, applied to $\mathrm{M}=T_{\varkappa(c)}^{1, *}$, produces an Azumaya algebra $\mathcal{A}_{c}:=\mathcal{A}_{\chi_{c}}$ on $\mathcal{M}_{\varkappa(c)}$. 
Assume now that $\varkappa(c)=c^{p}-c=0$, that is, the element $c \in \mathbb{k}$ is contained in the finite subfield $\mathbb{F}_{p} \subset \mathbb{k}$. Then, we apply the construction of subsection 4.1 to the open subset $\mathrm{M}:=\mathfrak{M}_{0}^{\mathrm{s}} \subset \mu^{-1}(0)$, of stable points. By Proposition 6.1.2, we obtain an Azumaya algebra $\mathcal{A}_{c}:=\mathcal{A}_{\chi_{c}}$ on the Frobenius twist of $\mathrm{Hilb}^{n} \mathbb{A}^{2}$, to be denoted $\mathrm{Hilb}^{(1)}$.

For any $c \in \mathbb{k}$, the Azumaya algebra $\mathcal{A}_{c}$ comes equipped with the canonical algebra homomorphism

$$
\Xi_{c}: \Gamma\left(\mathfrak{G}, \mathcal{D}_{\mathfrak{G}} / \mathcal{D}_{\mathfrak{G}} \cdot I_{c}\right)^{G} \stackrel{(4.5 .2)}{\longrightarrow} \Gamma\left(\mathcal{M}_{\varkappa(c)}^{(1)}, \mathcal{A}_{c}\right)
$$

6.3. A Harish-Chandra homomorphism for the Azumaya algebra. We are now going to construct a Harish-Chandra homomorphism for the Azumaya algebra $\mathcal{A}_{c}$.

Recall the open subset $U \subset \mathfrak{G}$ formed by the pairs $(x, v) \in \mathfrak{g}^{\mathrm{rs}} \times V$ such that $v$ is a cyclic vector for $x$.

Proposition 6.3.1. For any $c \in \mathbb{k}$, there is an algebra homomorphism $\Psi_{c}^{\mathcal{A}}$ making the following diagram commute:

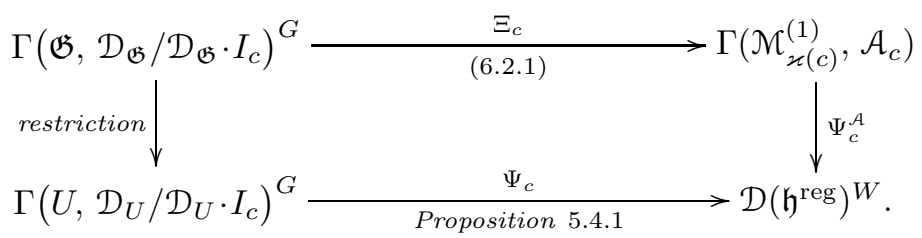

Proof. We only consider the most interesting case $\varkappa(c)=0$.

The action of $G$ on $U$ induces a Hamiltonian $G$-action on $T^{*} U$. Let $\mu_{U}: T^{*} U \rightarrow$ $\mathfrak{g}^{*}$ be the corresponding moment map, and set $\widetilde{U}:=\mu_{U}^{-1}(0)$. Since $U$ is an open subset of $\mathfrak{G}$, the map $\mu_{U}$ clearly equals the restriction of the moment map $\mu$ : $T^{*} \mathfrak{G} \longrightarrow \mathfrak{g}^{*}$ to the open subset $T^{*} U=\mathfrak{G}^{*} \times U \subset \mathfrak{G}^{*} \times \mathfrak{G}$. Thus, we have $\widetilde{U}=$ $\mu_{U}^{-1}(0)=\left(\mathfrak{G}^{*} \times U\right) \bigcap \mu^{-1}(0)$.

It is crucial for us that one has an open inclusion

$$
\widetilde{U} \subset \mathfrak{M}_{0}^{\mathrm{s}}=\mathrm{M}
$$

This trivially follows from definitions since a vector $v \in V$ which is cyclic for $x \in \mathfrak{g}$ is necessarily also cyclic for any pair of the form $(x, y) \in \mathfrak{g} \times \mathfrak{g}$.

Thus, we have the diagram

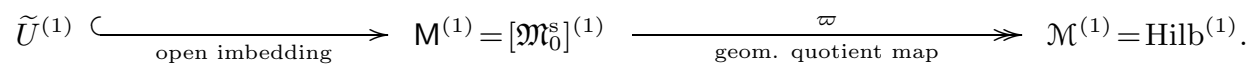

Restricting the sheaf $\mathcal{E}_{c}:=\mathcal{E}_{\chi_{c}}\left(\mathrm{cf} .(\underline{3.6 .3})\right.$ ) from $\left[\mathfrak{M}_{0}^{\mathrm{s}}\right]^{(1)}$ to $\widetilde{U}^{(1)}$ yields a $G^{(1)}$ equivariant algebra map $\Gamma\left(\left[\mathfrak{M}_{0}^{\mathrm{s}}\right]^{(1)}, \mathcal{E}_{c}\right) \longrightarrow \Gamma\left(\widetilde{U}^{(1)}, \mathcal{E}_{c}\right)$. Further, applying Lemma 3.6 .4 to $X:=U$ and $A:=G$, we get an algebra isomorphism

$$
\Gamma\left(U, \mathcal{D}_{U} / \mathcal{D}_{U} \cdot I_{c}\right)^{G} \cong \Gamma\left(\left[\mu_{U}^{-1}(0)\right]^{(1)}, \mathcal{E}_{c}\right)^{G^{(1)}}=\Gamma\left(\widetilde{U}^{(1)}, \mathcal{E}_{c}\right)^{G^{(1)}}
$$


By definition, we have $\mathcal{A}_{c}:=\varpi_{\bullet}\left(\left.\mathcal{E}_{c}\right|_{\left[\mathfrak{M}_{0}^{\mathrm{s}}\right]^{(1)}}\right)^{G^{(1)}}$. We obtain the following chain of algebra homomorphisms

$$
\begin{aligned}
& \Gamma\left(\mathcal{M}^{(1)}, \mathcal{A}_{c}\right)=\Gamma\left(\mathcal{M}^{(1)}, \varpi \cdot\left(\left.\mathcal{E}_{c}\right|_{\left[\mathfrak{M}_{0}^{\mathrm{s}}\right]^{(1)}}\right)^{G^{(1)}}\right) \\
& =\Gamma\left(\mathcal{M}^{(1)}, \varpi \cdot\left(\left.\mathcal{E}_{c}\right|_{\left[\mathfrak{M}_{0}^{\mathrm{s}}\right]^{(1)}}\right)\right)^{G^{(1)}}=\Gamma\left(\left[\mathfrak{M}_{0}^{\mathrm{s}}\right]^{(1)}, \mathcal{E}_{c}\right)^{G^{(1)}} \\
& \stackrel{\text { restriction }}{\longrightarrow} \Gamma\left(\widetilde{U}^{(1)}, \mathcal{E}_{c}\right)^{G^{(1)}} \underset{(6.3 .2)}{\stackrel{\sim}{\longrightarrow}} \Gamma\left(U, \mathcal{D}_{U} / \mathcal{D}_{U} \cdot I_{c}\right)^{G} \underset{\text { Prop. 5.4.1 }}{\stackrel{\Psi_{c}}{\longrightarrow}} \mathcal{D}\left(\mathfrak{h}^{\text {reg }}\right)^{W} \text {. }
\end{aligned}
$$

We let $\Psi_{c}^{\mathcal{A}}$ be the composite homomorphism. Commutativity of the diagram of the proposition is immediate from the construction above.

\section{Localization FUnCTOR FOR CheredniK Algebras}

7.1. From characteristic zero to characteristic $p$. We begin by recalling the general technique of transferring various results valid over fields of characteristic zero to similar results in characteristic $p$, provided $p$ is sufficiently large.

We fix $c=a / b \in \mathbb{Q}$ with $b>0$. For any prime $p>b$, reducing modulo $p$, we may (and will) treat $c=a / b$ as an element of $\mathbb{F}_{p}$. We let $\mathbb{k}_{p} \supset \mathbb{F}_{p}$ denote an algebraic closure of $\mathbb{F}_{p}$, and consider the corresponding $\mathbb{k}_{p}$-algebras $\mathrm{H}_{c}$ and $\mathrm{eH}_{c} \mathrm{e}$.

We begin with the following characteristic $p$ analogue of Proposition 5.4.4.

Lemma 7.1.1. Fix $c=a / b \in \mathbb{Q}$. For all sufficiently large primes $p$, we have an inclusion of $\mathbb{k}_{p}$-algebras $\mathrm{B}_{c} \subseteq \operatorname{Im}\left(\bar{\Psi}_{c}\right)$.

Proof. For $c=a / b \in \mathbb{Q}$, we consider the $\operatorname{ring} \mathbb{Z}\left[\frac{1}{n !}, c\right]=\mathbb{Z}\left[\frac{1}{n !}, \frac{1}{b}\right]$ obtained by inverting $n$ ! and $b$. The algebras $\Gamma\left(\mathfrak{G}, \mathcal{D}_{\mathfrak{G}} / \mathcal{D}_{\mathfrak{G}} \cdot I_{c}\right)^{G}$ and $\mathcal{D}\left(\mathfrak{h}^{\text {reg }}\right)^{W}$, are both defined over $\mathbb{Z}$, hence have natural $\mathbb{Z}\left[\frac{1}{n !}, c\right]$-integral structures. We will denote the corresponding $\mathbb{Z}\left[\frac{1}{n !}, c\right]$-algebras by the same symbols. Therefore, we may (and will) consider the $\mathbb{Z}\left[\frac{1}{n !}, c\right]$-integral version $\bar{\Psi}_{c}^{\mathbb{Z}}: \Gamma\left(\mathfrak{G}, \mathcal{D}_{\mathfrak{G}} / \mathcal{D}_{\mathfrak{G}} \cdot I_{c}\right)^{G} \longrightarrow \mathcal{D}\left(\mathfrak{h}^{\text {reg }}\right)^{W}$, of the homomorphism of Proposition 5.4.1. Thus, $\bar{\Psi}_{c}^{\mathbb{Z}}$ is a homomorphism of $\mathbb{Z}\left[\frac{1}{n !}, c\right]$-algebras.

Furthermore, we may choose a finite set of generators in the $\mathbb{Q}$-algebra $B_{c}$ in such a way that the $\mathbb{Z}\left[\frac{1}{n !}, c\right]$-subalgebra generated by this set is contained in the $\mathbb{Z}\left[\frac{1}{n !}, c\right]$-integral form of $\mathcal{D}\left(\mathfrak{h}^{\mathrm{reg}}\right)^{W}$. Denote this $\mathbb{Z}\left[\frac{1}{n !}, c\right]$-subalgebra by $\mathrm{B}_{c}^{\mathbb{Z}}$. Thus, we get a diagram of $\mathbb{Z}\left[\frac{1}{n !}, c\right]$-algebra maps

$$
\Gamma\left(\mathfrak{G}, \mathcal{D}_{\mathfrak{G}} / \mathcal{D}_{\mathfrak{G}} \cdot I_{c}\right)^{G} \longrightarrow \bar{\Psi}_{c}^{\mathbb{Z}} \longrightarrow \mathcal{D}\left(\mathfrak{h}^{\text {reg }}\right)^{W} \longleftrightarrow{ }^{j} \mathrm{~B}_{c}^{\mathbb{Z}},
$$

where $j$ denotes the inclusion.

Proposition 5.4 .4 says that $\mathbb{Q} \bigotimes_{\mathbb{Z}\left[\frac{1}{n !}, c\right]} \mathrm{B}_{c} \subseteq \mathbb{Q}_{\mathbb{Z}\left[\frac{1}{n !}, c\right]} \operatorname{Im}\left(\bar{\Psi}_{c}^{\mathbb{Z}}\right)$. Since all the algebras involved are finitely generated, it follows that there exists an integer $q \in \mathbb{Z}$ such that $\mathrm{B}_{c}^{\mathbb{Z}} \subset \operatorname{Im}\left(\bar{\Psi}_{c}^{\mathbb{Z}}\right)\left[\frac{1}{q}\right]$. Thus, for all primes $p>n$ which do not divide $q$, reducing the above inclusion modulo $p$, we get $\mathbb{k} \bigotimes_{\mathbb{Z}\left[\frac{1}{n !}, c\right]} \mathrm{B}_{c} \subseteq \mathbb{k} \bigotimes_{\mathbb{Z}\left[\frac{1}{n !}, c\right]} \operatorname{Im}\left(\bar{\Psi}_{c}^{\mathbb{Z}}\right)$. The lemma is proved.

We now consider the algebras $\mathrm{H}_{c}$ and $\mathrm{eH}_{c}$ e over the ground field $\mathbb{Q}$ of the rational numbers, and let $\mathrm{H}_{c}$ eH $\mathrm{H}_{c}$ be the two-sided ideal in $\mathrm{H}_{c}$ generated by the idempotent e.

We will use the following result from GS. 
Proposition 7.1.2. For any $c \in \mathbb{Q}^{\text {good }}$ (see (1.3.1)), we have $\mathrm{H}_{c}=\mathrm{H}_{c} \mathrm{eH}$. Thus, the $\mathrm{eH}_{c} \mathrm{e}-\mathrm{H}_{c}$-bimodule $\mathrm{eH}_{c}$ provides a Morita equivalence between the algebras $\mathrm{H}_{c}$ and $\mathrm{eH}_{c} \mathrm{e}$.

We are going to deduce a similar result in characteristic $p$, which reads:

Corollary 7.1.3. Given $c \in \mathbb{Q}^{\text {good }}$, there exists a constant $d=d(c)$ such that for all primes $p>d(c)$, the $\mathrm{eH}_{c} \mathrm{e}-\mathrm{H}_{c}$-bimodule $\mathrm{eH}_{c}$ provides a Morita equivalence between the $\mathbb{k}_{p}$-algebras $\mathrm{H}_{c}$ and $\mathrm{eH}_{c} \mathrm{e}$.

Remark 7.1.4. We emphasize that, in this corollary and in various other results below, a rational value of the parameter $c$ must be fixed first. The choice of $c$ dictates a lower bound $d(c)$ for allowed primes $p$, and only after that one considers the corresponding Cherednik algebras over $\mathbb{k}_{p}$. Thus, if $c \in \mathbb{Q}$ and $p$ have been chosen as above, and $c^{\prime} \in \mathbb{Q}$ is such that $c^{\prime}=c \bmod p$, then $\mathbf{H}_{c} \cong \mathbf{H}_{c^{\prime}}$ as $\mathbb{k}_{p^{-}}$ algebras; yet, it is quite possible that we have $d(c)<p<d\left(c^{\prime}\right)$, hence, the results of this section do not apply for $\mathrm{H}_{c^{\prime}}$ viewed as a $\mathbb{k}_{p}$-algebra.

The proof of the corollary will exploit the following standard result of commutative algebra; Gr, Expose IV, Lemma 6.7.

Generic Flatness Lemma. Let $A$ be a commutative noetherian integral domain, $B$ a (commutative) A-algebra of finite type, and $M$ a finitely generated $B$-module. Then, there is a nonzero element $f \in A$ such that $M_{(f)}$, the localization of $M$, is a free $A_{(f)}$-module.

Proof of Corollary 7.1 .3 . Let $\mathbf{c}$ denote an independent variable, and let $\mathbb{Z}\left[\frac{1}{n !}\right][\mathbf{c}]$, be the localization of the polynomial ring $\mathbb{Z}[\mathbf{c}]$ at the number $n ! \in \mathbb{Z}$. Given a nonzero element $f \in \mathbb{Z}\left[\frac{1}{n !}\right][\mathbf{c}]$ and a $\mathbb{Z}\left[\frac{1}{n !}\right][\mathbf{c}]$-module $M$, we write $M_{(f)}$ for the localization of $M$ at $f$, a module over the localized ring $\mathbb{Z}\left[\frac{1}{n !}\right][\mathbf{c}]_{(f)}$.

Let $\mathrm{H}$ and eHe be the universal Cherednik algebras, viewed as algebras over the ground ring $\mathbb{Z}\left[\frac{1}{n !}\right][\mathbf{c}]$. We first establish the following.

Claim 7.1.5. There exists a polynomial $f \in \mathbb{Z}\left[\frac{1}{n !}\right][\mathbf{c}]$ such that $\mathrm{H}_{(f)}=(\mathrm{HeH})_{(f)}$ and, moreover, such that $f(c) \neq 0$ for any $c \in \mathbb{Q}^{\text {good }}$.

To prove the claim, consider the standard increasing filtration $F$. on $\mathrm{H}$ and the induced filtration $F \cdot(\mathrm{H} / \mathrm{HeH})$ on the quotient algebra $\mathrm{H} / \mathrm{HeH}_{2} 2$ The associated graded $\operatorname{gr}^{F}(\mathrm{H} / \mathrm{HeH})$, is a finitely generated $\mathbb{Z}\left[\frac{1}{n !}\right][\mathbf{c}]$-algebra. Clearly, it suffices to show that this algebra vanishes generically over $\operatorname{Spec} \mathbb{Z}\left[\frac{1}{n !}\right][\mathbf{c}]$. To this end, observe that $\operatorname{gr}(\mathrm{H} / \mathrm{HeH})$ is a finitely generated module over the graded algebra $\operatorname{gr}(\mathrm{H})$, which is a quotient of the smash-product algebra

$$
\mathbb{Z}\left[\frac{1}{n !}\right][\mathbf{c}]\left[\mathfrak{h} \times \mathfrak{h}^{*}\right] \ltimes \mathbb{Z}\left[\frac{1}{n !}\right][\mathbf{c}]\left[S_{n}\right]
$$

(here we regard $\mathfrak{h}$ as a free rank $n$ module over $\mathbb{Z}\left[\frac{1}{n !}\right][\mathbf{c}]$; its dual $\mathfrak{h}^{*}$ is also free of rank $n$; by the Poincare-Birkhoff-Witt theorem proved in [EG], the algebra $\operatorname{gr}(\mathrm{H})$ and the above smash-product become isomorphic after tensoring with $\mathbb{Q}$ ). We deduce that $\operatorname{gr}(\mathrm{H} / \mathrm{HeH})$ is a finitely generated module over the commutative algebra $\mathbb{Z}\left[\frac{1}{n !}\right][\mathbf{c}]\left[\mathfrak{h} \times \mathfrak{h}^{*}\right]$.

The Generic Flatness Lemma implies that there exists a nonzero polynomial $f \in \mathbb{Z}\left[\frac{1}{n !}\right][\mathbf{c}]$ such that $\operatorname{gr}(\mathrm{H} / \mathrm{HeH})_{(f)}$ is free over $\mathbb{Z}\left[\frac{1}{n !}\right][\mathbf{c}]_{(f)}$. On the other hand, for

\footnotetext{
${ }^{2}$ The argument below is similar to $\mathrm{Q}$.
} 
$c \in \mathbb{Q}^{\text {good }}$, by Proposition 7.1.2 we have $\mathbb{Q} \otimes \mathrm{H}_{c}=\mathbb{Q} \otimes\left(\mathrm{H}_{c} \mathrm{eH}\right)$; that is, the fiber of $\mathbb{Q} \otimes \operatorname{gr}(\mathrm{H} / \mathrm{HeH})$ over $c \in \mathbb{Q}^{\text {good }}$ vanishes. We conclude that $\mathrm{H}=\mathrm{HeH}$ holds over a nonempty Zariski open subset of $\operatorname{Spec}\left(\mathbb{Z}\left[\frac{1}{n !}\right][\mathbf{c}]\right)$ that has a nontrivial intersection with any closed subscheme $\{\mathbf{c}=c\}, c \in \mathbb{Q}^{\text {good }}$. This proves Claim 7.1.5.

We complete the proof of Corollary 7.1 .3 as follows. By Claim 7.1.5, there exist a polynomial $f \in \mathbb{Z}\left[\frac{1}{n !}\right][\mathbf{c}]$ and elements $h_{i}^{\prime}, h_{i}^{\prime \prime} \in \mathrm{H}, i=1, \ldots, m$, such that

$$
f \cdot \mathbf{1}_{\mathrm{H}}=\sum_{i=1}^{m} h_{i}^{\prime} \cdot \mathrm{e} \cdot h_{i}^{\prime \prime} \text { holds in } \mathbf{H},
$$

where $\mathbf{1}_{\mathrm{H}}$ denotes the unit of the $\mathbb{Z}\left[\frac{1}{n !}\right][\mathbf{c}]$-algebra $\mathrm{H}$. We may specialize this equation at any rational value $c=a / b \in \mathbb{Q}$ to obtain a similar equation for the corresponding $\mathbb{Z}\left[\frac{1}{n !}, \frac{1}{b}\right]$-algebras. If $c \in \mathbb{Q}^{\text {good }}$, then, according to Claim 7.1.5, we may further assume that $f(c)=k / l \neq 0$.

Now, let $p$ be a prime such that $p>\max \{n, k, l\}$. Reducing (the specialization at $c$ of) equation (7.1.6) modulo $p$, for the corresponding $\mathbb{F}_{p}$-algebras we get $f(c) \cdot \mathbf{1}_{\mathrm{H}_{c}}=$ $\sum_{i=1}^{m} h_{i}^{\prime} \cdot \mathrm{e} \cdot h_{i}^{\prime \prime}$. Thus $f(c)=k / l$ is a nonzero, hence, invertible element in $\mathbb{F}_{p}$ and, since $\mathbb{F}_{p} \subset \mathbb{k}_{p}$, in the $\mathbb{k}_{p}$-algebra $\mathrm{H}_{c}$ we obtain $\mathbf{1}_{\mathrm{H}_{c}}=\frac{1}{f(c)} \sum_{i=1}^{m} h_{i}^{\prime} \cdot \mathrm{e} \cdot h_{i}^{\prime \prime}$.

Thus, we have proved that $\mathbf{1}_{\mathrm{H}_{c}} \in \mathrm{H}_{c} \mathrm{e} \mathrm{H}_{c}$, and the first statement of the corollary follows. It is well known that this implies the last statement of the corollary as well.

7.2. Localization of the spherical subalgebra. We have the following $\mathcal{A}_{c^{-}}$ version of Proposition 5.4.4.

Theorem 7.2.1. Fix $c=a / b \in \mathbb{Q}$. For all sufficiently large primes $p$, we have:

The image of the Harish-Chandra homomorphism $\Psi_{c}^{\mathcal{A}}$ of Proposition 6.3 .1 is equal to the subalgebra $\mathrm{B}_{c} \subset \mathcal{D}\left(\mathfrak{h}^{\text {reg }}\right)^{W}$ (algebras over $\mathbb{k}_{p}$ ). Moreover, the resulting map gives an algebra isomomorphism $\Psi_{c}^{\mathcal{A}}: \Gamma\left(\mathrm{Hilb}^{(1)}, \mathcal{A}_{c}\right) \stackrel{\sim}{\longrightarrow} \mathrm{B}_{c}$.

Proof of this theorem will be given later in this section.

Remark 7.2.2. A similar construction also produces an isomorphism $\Psi_{c}^{\mathcal{A}}: \Gamma\left(\mathcal{M}_{\varkappa(c)}^{(1)}\right.$, $\left.\mathcal{A}_{c}\right) \stackrel{\sim}{\longrightarrow} \mathrm{B}_{c}$, for all $c \in \mathbb{k}_{p}$ (not only for $c \in \mathbb{F}_{p}$ ). The proof of this generalization is similar to the proof of Theorem 7.2.1, but involves twisted differential operators and twisted cotangent bundles. It will be presented elsewhere.

Composing the isomorphism of Theorem 7.2.1 with the inverse of the Dunkl representation (5.2.1), we obtain the following Azumaya version of the Spherical Harish-Chandra isomorphism considered in EG],

$$
\Phi_{c}^{\mathcal{A}}: \Gamma\left(\operatorname{Hilb}^{(1)}, \mathcal{A}_{c}\right) \stackrel{\Psi_{c}^{\mathcal{A}}}{\longrightarrow} \mathrm{B}_{c} \stackrel{\left(\Theta_{c}\right)^{-1}}{\sim} \mathrm{eH}_{c} \mathrm{e} .
$$

Thus, we have proved part (i) of the following theorem, which is one of the main results of the paper

Theorem 7.2.4. Fix $c=a / b \in \mathbb{Q}$. Then there exists a constant $d=d(c)$ such that for all primes $p>d(c)$, we have:

(i) The composite morphism in (7.2.3) yields a $\mathbb{k}_{p}$-algebra isomorphism $\Phi_{c}^{\mathcal{A}}$ : $\mathrm{eH}_{c} \mathrm{e} \stackrel{\sim}{\longrightarrow} \Gamma\left(\mathrm{Hilb}^{(1)}, \mathcal{A}_{c}\right)$.

(ii) $H^{i}\left(\operatorname{Hilb}^{(1)}, \mathcal{A}_{c}\right)=0$ for all $i>0$. 
(iii) If $c \in \mathbb{Q}^{\text {good }}$, then the (derived) global sections functor $\mathrm{R} \Gamma: D^{b}\left(\mathcal{A}_{c^{-}} \mathrm{Mod}\right) \rightarrow$ $D^{b}\left(\mathrm{eH}_{c} \mathrm{e}-\mathrm{Mod}\right)$ is an equivalence of bounded derived categories.

Proof. Part (i) follows from Theorem 7.2.1 (to be proved below), and part (ii) is a consequence of Theorem 4.1.4(ii). We are going to deduce part (iii) of Theorem 7.2.4 from Theorem 4.1.4(iii). To do so, we need to know that the algebra $\mathrm{eH}_{c} \mathrm{e} \cong \Gamma\left(\mathrm{Hilb}^{(1)}, \mathcal{A}_{c}\right)$ has finite homological dimension. But this follows from the Morita equivalence of Corollary 7.1.3, since the algebra $\mathrm{H}_{c}$ is known to have finite homological dimension, which is equal to $2 n$; cf. [EG].

Proof of Theorem [7.2.1. Fix $c \in \mathbb{Q}$, and let $p \gg 0$ be such that Lemma 7.1.1 holds for $p$.

Recall an increasing filtration on the algebra $\Gamma\left(\operatorname{Hilb}^{(1)}, \mathcal{A}_{c}\right)$, introduced in subsection 4.2. such that for the associated graded algebra we have $\operatorname{gr}^{\mathcal{R}} \Gamma\left(\operatorname{Hilb}^{(1)}, \mathcal{A}_{c}\right)$ $=\Gamma\left(\mathrm{Hilb}^{n} \mathbb{A}^{2}, \mathcal{O}_{\mathrm{Hilb}^{n} \mathbb{A}^{2}}\right)$; see Proposition 4.3.3(ii).

We consider the commutative diagram of Proposition 6.3.1. All maps in that diagram are filtration preserving, and the corresponding commutative diagram of associated graded maps reads:

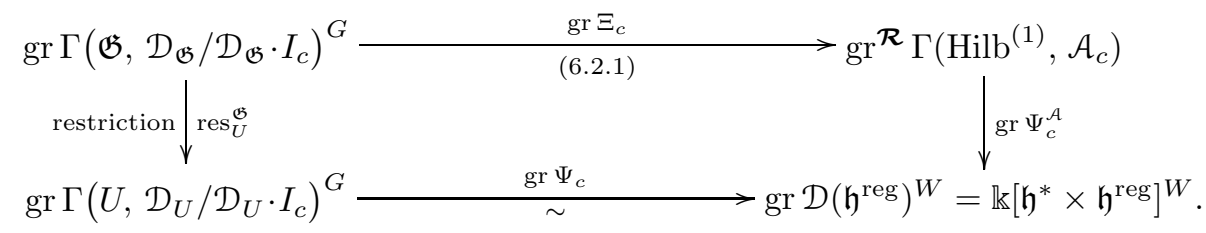

Furthermore, we have the following graded algebra isomorphisms:

$$
\operatorname{gr}^{\mathcal{R}} \Gamma\left(\mathrm{Hilb}^{(1)}, \mathcal{A}_{c}\right) \stackrel{\text { Proposition }[4.3 .3 \mathrm{ii})}{\sim} \Gamma\left(\mathrm{Hilb}^{n} \mathbb{A}^{2}, \mathcal{O}_{\mathrm{Hilb}^{n} \mathbb{A}^{2}}\right) \stackrel{(6.1 .4)}{\sim} \mathbb{k}\left[\mathfrak{h}^{*} \times \mathfrak{h}\right]^{W} .
$$

Moreover, by going through definitions, it is easy to verify that the composite map in (7.2.6) is equal to the map gr $\Psi_{c}^{\mathcal{A}}$ in (7.2.5). It follows, in particular, that $\Psi_{c}^{\mathcal{A}}$ is an injective morphism.

On the other hand, Lemma 7.1.1 and Proposition 6.3.1 yield

$$
\mathrm{B}_{c} \subset \operatorname{Im}\left(\bar{\Psi}_{c}\right):=\Psi_{c}\left(\operatorname{res}_{U}^{\mathfrak{G}}\left(\Gamma\left(\mathfrak{G}, \mathcal{D}_{\mathfrak{G}} / \mathcal{D}_{\mathfrak{G}} \cdot I_{c}\right)^{G}\right)\right) \subseteq \operatorname{Im}\left(\Psi_{c}^{\mathcal{A}}\right)
$$

Hence, using commutativity of diagram (7.2.5), we obtain the following commutative diagram of graded algebra morphisms:

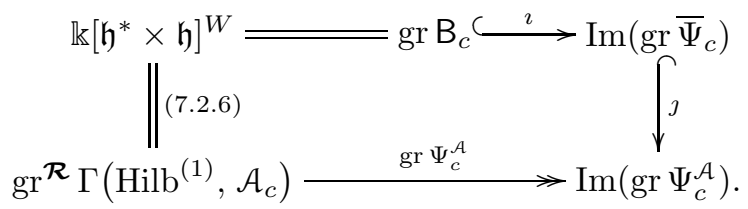

We deduce from commutativity of diagram (7.2.8) that both $\imath$ and $\jmath$ must be surjective and, therefore, $\operatorname{Im}\left(\operatorname{gr} \Psi_{c}^{\mathcal{A}}\right)=\operatorname{gr} B_{c}$. Now, the inclusions in (7.2.7) show that we must have $\operatorname{Im}\left(\Psi_{c}^{\mathcal{A}}\right)=\mathrm{B}_{c}$ and, moreover, the map $\Psi_{c}^{\mathcal{A}}$ gives an isomorphism $\Gamma\left(\operatorname{Hilb}^{(1)}, \mathcal{A}_{c}\right) \stackrel{\sim}{\longrightarrow} \mathrm{B}_{c}$. 
7.3. Localization of the algebra $\mathrm{H}_{c}$. We introduce the following localization functor $\mathcal{L} o c: \quad N \mapsto \mathcal{A}_{c} \stackrel{L}{\otimes_{\mathrm{eH}} \mathrm{e}} N$, which is the left adjoint to the functor $\mathrm{R} \Gamma$ : $D^{b}\left(\mathcal{A}_{c^{-}} \mathrm{Mod}\right) \rightarrow D^{b}\left(\mathrm{eH}_{c} \mathrm{e}-\mathrm{Mod}\right)$. Since $\mathrm{R} \Gamma\left(\mathcal{A}_{c}\right)=\mathrm{eH}_{c} \mathrm{e}$, and the functor $\mathrm{R} \Gamma$ is an equivalence by Theorem [7.2.4 we conclude that $\mathcal{L} o c\left(\mathrm{eH}_{c} \mathrm{e}\right)=\mathcal{A}_{c}$ and, moreover, the functor $\mathcal{L} o c$ is also an equivalence which is a quasi-inverse to $\mathrm{R} \Gamma(-)$.

Observe next that $\mathrm{eH}_{c}$ is a projective $\mathrm{eH}_{c} \mathrm{e}$-module, by Corollary [7.1.3. Hence we conclude that $\mathcal{R}_{c}:=\operatorname{Loc}\left(\mathrm{eH}_{c}\right)=\mathcal{A}_{c} \otimes_{\mathrm{eH}} \mathrm{e} \mathrm{eH}_{c}$ is a locally free sheaf of $\mathcal{A}_{c^{-}}$ modules. Moreover, it is easy to see by looking at the restrictions of the associated graded modules to the generic locus of $(\mathfrak{h} \times \mathfrak{h}) / W$ that the rank of $\mathrm{eH}_{c}$ viewed as a projective $\mathrm{eH}_{c} \mathrm{e}$-module equals $n$ !. Therefore, we deduce that $\mathcal{R}_{c}$ is a vector bundle on Hilb ${ }^{(1)}$ of rank $n ! \cdot p^{2 n}$.

We put $\mathcal{H}_{c}:=\mathcal{E} n d_{\mathcal{A}_{c}}\left(\mathcal{R}_{c}\right)$. This is clearly an Azumaya algebra on Hilb ${ }^{(1)}$ again, and the degree of this Azumaya algebra is equal to $n ! \cdot p^{2 n}$. Furthermore, the left $\mathrm{H}_{c}$-action on each fiber of the sheaf $\mathcal{R}_{c}$ induces a natural algebra map

$$
\mathrm{H}_{c} \longrightarrow \Gamma\left(\operatorname{Hilb}^{(1)}, \mathcal{H}_{c}\right)
$$

The second main result of the paper reads:

Theorem 7.3.2. Fix $c \in \mathbb{Q}^{\text {good }}$. Then, there exists a constant $d=d(c)$ such that for all primes $p>d(c)$, we have:

(i) The map (7.3.1) is an algebra isomorphism, moreover, $\mathrm{R}^{i}\left(\mathrm{Hilb}^{(1)}, \mathcal{H}_{c}\right)=0$, $\forall i>0$.

(ii) The functor $\mathrm{R} \Gamma: D^{b}\left(\mathcal{H}_{c}-\mathrm{Mod}\right) \rightarrow D^{b}\left(\mathrm{H}_{c}\right.$-Mod) is a triangulated equivalence.

Proof. We have

$$
\begin{aligned}
\mathrm{R}^{i}\left(\operatorname{Hilb}^{(1)}, \mathcal{H}_{c}\right) & \cong \operatorname{R}^{i}\left(\operatorname{Hilb}^{(1)}, \mathcal{E} n d_{\mathcal{A}}\left(\mathcal{R}_{c}\right)\right) \cong \operatorname{Ext}_{D^{b}\left(\mathcal{A}_{c}-\mathrm{Mod}\right)}^{i}\left(\mathcal{R}_{c}, \mathcal{R}_{c}\right) \\
& \underset{\text { Theorem[7.2.4 }}{\sim} \operatorname{Ext}_{D^{b}\left(\mathrm{eH}_{c} \mathrm{e}-\mathrm{Mod}\right)}\left(\mathrm{eH}_{c}, \mathrm{eH}_{c}\right) .
\end{aligned}
$$

The Ext-group on the right vanishes for all $i>0$ since $\mathrm{eH}_{c}$ is a projective $\mathrm{eH}_{c} \mathrm{e}-$ module. This proves the vanishing statement in part (i). The statement of part (i) for $i=0$ follows from the isomorphisms:

$$
\mathrm{H}_{c} \underset{\text { EG Theorem 1.5(iv)] }}{\sim} \operatorname{Hom}_{\mathrm{eH}_{c} \mathrm{e}}\left(\mathrm{eH}_{c}, \mathrm{eH}_{c}\right) \stackrel{(7.3 .3)}{\sim} \Gamma\left(\operatorname{Hilb}^{(1)}, \mathcal{H}_{c}\right) .
$$

To prove part (ii) we use a commutative diagram:

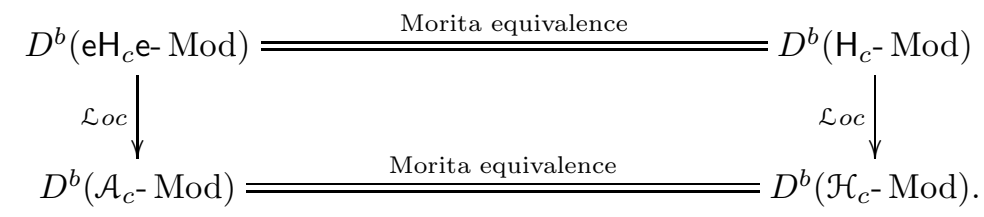

Since the left vertical arrow is an equivalence by Theorem 7.2 .4 , it follows that the right vertical arrow is an equivalence as well. The functor $\mathrm{R} \Gamma$ is a right adjoint of $\mathcal{L} o c$, hence, it must also be an equivalence, which is a quasi-inverse of $\mathcal{L} o c$. 
7.4. Splitting on the fibers of the Hilbert-Chow map. We have the HilbertChow map $\Upsilon: \operatorname{Hilb}^{n} \mathbb{A}^{2} \rightarrow(\mathfrak{h} \times \mathfrak{h}) / W$. Given $\xi \in(\mathfrak{h} \times \mathfrak{h}) / W$, write Hilb $\mathrm{H}_{\xi}:=\Upsilon^{-1}(\xi)$ for the fiber of $\Upsilon$ over $\xi$, and let $\widehat{\mathrm{Hilb}}_{\xi}^{(1)}$ denote the completion of Hilb ${ }^{(1)}$ along this fiber, a formal scheme.

The next result is essentially due to $\mathrm{BK}]$.

Theorem 7.4.1. For any point $\xi \in(\mathfrak{h} \times \mathfrak{h}) / W$, the restriction of the Azumaya algebra $\mathcal{H}_{c}$, resp. $\mathcal{A}_{c}$, to the formal neighborhood of the fiber $\mathrm{Hilb}_{\xi}^{(1)} \subset \mathrm{Hilb}^{(1)}$ splits, i.e., there is a vector bundle $\mathcal{V}_{c, \xi}$, resp. $\mathcal{W}_{c, \xi}$, on $\widehat{\operatorname{Hilb}}_{\xi}^{(1)}$ such that one has

$$
\left.\mathcal{H}_{c}\right|_{\widehat{\mathrm{Hilb}}_{\xi}^{(1)}} \cong \mathcal{E} n d_{\mathcal{O}_{\mathrm{Hib}_{\xi}^{(1)}}} \mathcal{V}_{c, \xi}, \quad \text { resp. },\left.\quad \mathcal{A}_{c}\right|_{\widehat{\mathrm{Hilb}}_{\xi}^{(1)}} \cong \mathcal{E} n d_{\mathcal{O}_{\mathrm{Hib}_{\xi}^{(1)}}} \mathcal{W}_{c, \xi}
$$

The above vector bundle $\mathcal{V}_{c, \xi}$, resp. $\mathcal{W}_{c, \xi}$, is called a splitting bundle for $\mathcal{H}_{c}$, resp. for $\mathcal{A}_{c}$.

Corollary 7.4.2. For any $\xi \in(\mathfrak{h} \times \mathfrak{h}) / W$ and $i>0$, we have $\operatorname{Ext}^{i}\left(\mathcal{V}_{c, \xi}, \mathcal{V}_{c, \xi}\right)=0$, resp., $\operatorname{Ext}^{i}\left(\mathcal{W}_{c, \xi}, \mathcal{W}_{c, \xi}\right)=0$, where the Ext-groups are considered in the category $\operatorname{Coh}\left(\widehat{\operatorname{Hilb}}_{\xi}^{(1)}\right)$.

The rank of a splitting bundle is equal to the degree of the corresponding Azumaya algebra. In particular, we have $\operatorname{rk} \mathcal{W}_{c, \xi}=p^{n}$ and $\operatorname{rk} \mathcal{V}_{c, \xi}=p^{n} \cdot n$ !. This suggests the following:

Conjecture 7.4.3. For any $\xi \in[(\mathfrak{h} \times \mathfrak{h}) / W]^{(1)}$, there is a vector bundle isomorphism $\mathrm{Fr}^{*} \mathcal{V}_{c, \xi} \cong\left(\operatorname{Fr}^{*} \mathcal{W}_{c, \xi}\right) \otimes\left(\left.\mathcal{P}\right|_{\widehat{\mathrm{Hilb}}_{\mathrm{Fr}(\xi)}^{n}}\right)$, where $\mathcal{P}$ denotes the Procesi bundle on Hilb ${ }^{n} \mathbb{A}^{2} ;$ see $[\mathrm{H}$.

Remark. In view of Theorem 4.1.4(iii) it is sufficient to prove the Conjecture for $c=0$, that is, for the case where $\mathrm{H}_{c}=\mathcal{D}(\mathfrak{h}) \# W$.

Proof of Theorem 7.4.1. Clearly, it suffices to prove the theorem for $\mathcal{A}_{c}$. We repeat the argument in the proof of [BK, Proposition 5.4].

First, recall that Morita equivalence classes of Azumaya algebras on a scheme $Y$ are classified by $\operatorname{Br}(Y)$, the Brauer group of $Y$. Furthermore, the Brauer group of a local complete $\mathbb{k}$-algebra is known to be trivial. Thus, proving the theorem amounts to showing that, for any $c \in \mathbb{F}_{p}$, the class $\left[\mathcal{A}_{c}\right] \in B r\left(\operatorname{Hilb}^{(1)}\right)$ belongs to the image of the pull-back morphism $\Upsilon^{*}: \operatorname{Br}\left([(\mathfrak{h} \times \mathfrak{h}) / W]^{(1)}\right) \rightarrow B r\left(\operatorname{Hilb}^{(1)}\right)$.

To prove this, we consider the following diagram:

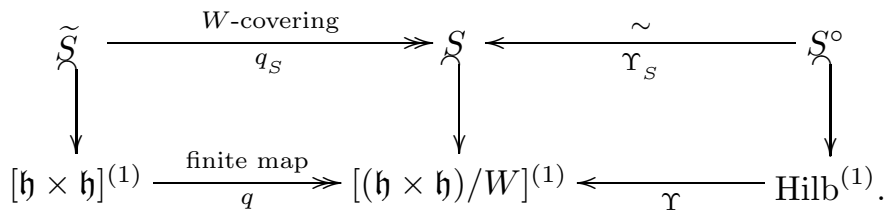

In this diagram, $S$ is a Zariski open dense subset in $[(\mathfrak{h} \times \mathfrak{h}) / W]^{(1)}$ such that:

- The Hilbert-Chow map restricts to an isomorphism $S^{\circ}:=\Upsilon^{-1}(S) \stackrel{\sim}{\longrightarrow} S$, to be denoted $\Upsilon_{S}$, and

- The projection $q:[\mathfrak{h} \times \mathfrak{h}]^{(1)} \longrightarrow[(\mathfrak{h} \times \mathfrak{h}) / W]^{(1)}$ is unramified over $S$. Thus, we have a Galois covering $\widetilde{S}:=q^{-1}(S) \rightarrow S$, to be denoted $q_{S}$. 
Our goal is to construct a class $\beta \in \operatorname{Br}\left([(\mathfrak{h} \times \mathfrak{h}) / W]^{(1)}\right)$ such that $\left[\mathcal{A}_{c}\right]=\Upsilon^{*}(\beta)$. We will follow the strategy of $[\mathrm{BK}]$.

Let $Y$ be an arbitrary affine scheme, and let $H_{e t}^{2}\left(Y, \mathbb{G}_{\mathbf{m}}\right)$ torsion be the torsion subgroup of the second étale cohomology of $Y$ with coefficients in the multiplicative group. By a theorem of Gabber [Ga], one has an isomorphism $\operatorname{Br}(Y) \cong$ $H_{e t}^{2}\left(Y, \mathbb{G}_{\mathbf{m}}\right)_{\text {torsion. }}$ Furthermore, it is a simple matter to see that the norm-map associated to the projection $q:[\mathfrak{h} \times \mathfrak{h}]^{(1)} \longrightarrow[(\mathfrak{h} \times \mathfrak{h}) / W]^{(1)}$ gives rise to a morphism on étale cohomology; cf. [BK]:

$$
\left.q_{*}: H_{\text {et }}^{2}\left([\mathfrak{h} \times \mathfrak{h}]^{(1)}, \mathbb{G}_{\mathbf{m}}\right)_{\text {torsion }}^{W} \longrightarrow H_{\text {et }}^{2}((\mathfrak{h} \times \mathfrak{h}) / W), \mathbb{G}_{\mathbf{m}}\right)_{\text {torsion }} .
$$

Now let $\mathcal{D}^{(1)}:=\operatorname{Fr} . \mathcal{D}_{\mathfrak{h}}$ be the standard Azumaya algebra on $T^{*} \mathfrak{h}^{(1)}=[\mathfrak{h} \times \mathfrak{h}]^{(1)}$, arising from the sheaf of crystalline differential operators on $\mathfrak{h}$, cf. $\S 2$. The sheaf $\mathcal{D}^{(1)}$ has a natural $W$-equivariant structure, hence the corresponding class $\left[\mathcal{D}^{(1)}\right]$ is a $W$-invariant class in the Brauer group, that is an element of $\operatorname{Br}\left([\mathfrak{h} \times \mathfrak{h}]^{(1)}\right)^{W} \cong$ $H_{\text {et }}^{2}\left([\mathfrak{h} \times \mathfrak{h}]^{(1)}, \mathbb{G}_{\mathbf{m}}\right)_{\text {torsion }}^{W}$. We set

$$
\beta:=q_{*}\left(\left[\mathcal{D}^{(1)}\right]\right) \in H_{e t}^{2}\left([(\mathfrak{h} \times \mathfrak{h}) / W]^{(1)}, \mathbb{G}_{\mathbf{m}}\right)_{\text {torsion }} \cong \operatorname{Br}\left([(\mathfrak{h} \times \mathfrak{h}) / W]^{(1)}\right) .
$$

The theorem would follow provided we show that $\Upsilon^{*} \beta=\left[\mathcal{A}_{c}\right]$. We first prove a weaker claim:

$$
\left.\left(\Upsilon^{*} \beta\right)\right|_{S^{\circ}}=\left.\left[\mathcal{A}_{c}\right]\right|_{S^{\circ}} \text { holds in } \operatorname{Br}\left(S^{\circ}\right)
$$

To see this, restrict the Azumaya algebra $\mathcal{D}^{(1)}$ to the open subset $\widetilde{S} \subset[\mathfrak{h} \times \mathfrak{h}]^{(1)}$. The map $q_{S}$ (see (7.4.4) $)$ is a Galois covering with the Galois group $W$. It follows that the sheaf $\mathcal{B}:=\left(\left.\left(q_{S}\right) \cdot\left(\mathcal{D}^{(1)}\right)\right|_{\widetilde{S}}\right)^{W}$ is an Azumaya algebra on $S$. Furthermore, it is immediate from the construction that, in $\operatorname{Br}(S)$, one has an equality $\left.\beta\right|_{S}=[\mathcal{B}]$. Pulling back via the isomorphism $\Upsilon_{S}$ (see (17.4.4) $)$, we deduce that $\left.\left(\Upsilon^{*} \beta\right)\right|_{S^{\circ}}=$ $\left(\Upsilon_{S}\right)^{*}\left(\left.\beta\right|_{S}\right)=\left(\Upsilon_{S}\right)^{*}[\mathcal{B}]$

To complete the proof of (7.4.5), we use Theorem 4.1.4(iv) and deduce that, for all $c \in \mathbb{F}_{p}$, the corresponding Azumaya algebras $\mathcal{A}_{c}$ are Morita equivalent, hence represent the same class in $\operatorname{Br}\left(\mathrm{Hilb}^{(1)}\right)$. Thus, we may assume, without loss of generality, that $c=0$. In that case the corresponding algebra $\mathrm{eH}_{c} \mathrm{e}$ is isomorphic to $\mathcal{D}(\mathfrak{h})^{W}$. Furthermore, going through the Hamiltonian reduction construction of the Azumaya algebra $\mathcal{A}_{0}$, it is easy to verify that we have an Azumaya algebra

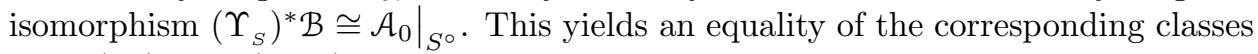
in $\operatorname{Br}\left(S^{\circ}\right)$, and (7.4.5) follows.

To complete the proof of the theorem we recall the well-known result saying that restriction to a Zariski open dense subset induces an injective morphism of the corresponding Brauer groups. Thus, we have an injection $\operatorname{Br}\left(\mathrm{Hilb}^{(1)}\right) \hookrightarrow$ $\operatorname{Br}\left(S^{\circ}\right),\left.\alpha \mapsto \alpha\right|_{S^{\circ}}$, and we have shown above that $\left.\left(\Upsilon^{*} \beta\right)\right|_{S^{\circ}}=\left.\left[\mathcal{A}_{0}\right]\right|_{S^{\circ}}$. Hence, $\Upsilon^{*} \beta=\left[\mathcal{A}_{0}\right]=\left[\mathcal{A}_{c}\right], \forall c \in \mathbb{F}_{p}$, and the theorem is proved.

7.5. On $t$-structures. Fix $c \in \mathbb{Q}^{\text {good }}$ and a large enough prime $p$.

In subsection 4.4, we have constructed a canonical $\mathcal{A}_{c+1}-\mathcal{A}_{c}$-bimodule ${ }_{c+1} \mathcal{A}_{c}$ that provides Morita equivalence of the Azumaya algebras $\mathcal{A}_{c}$ and $\mathcal{A}_{c+1}$. We define the following geometric shift functor:

$$
S_{+}: D^{b}\left(\mathcal{A}_{c^{-}} \mathrm{Mod}\right) \longrightarrow D^{b}\left(\mathcal{A}_{c+1^{-}} \operatorname{Mod}\right), \quad \mathcal{M} \longmapsto S_{+}(\mathcal{M})={ }_{c+1} \mathcal{A}_{c} \otimes_{\mathcal{A}_{c}} \mathcal{M}
$$


We consider the following diagram of triangulated functors:

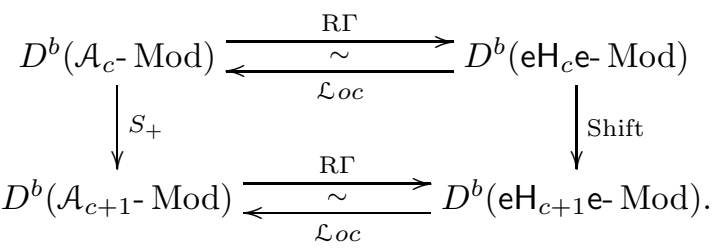

Here, the horizontal arrows are triangulated equivalences by the localization Theorem 7.2.4. The functor $S_{+}$on the left is an equivalence by definition. The shift functor on the right of the diagram was constructed in BEG, and it was shown to be an equivalence for any nonnegative $c \in \mathbb{Q}^{\text {good }}$ in GS. We will prove in a subsequent publication [FG] that diagram (7.5.1) commutes.

In any case, for each $c \in \mathbb{Q}^{\text {good }}$, we consider the $t$-structure on $D^{b}\left(\mathcal{A}_{c^{-}}\right.$Mod) obtained by transporting the natural $t$-structure (with abelian core $\mathrm{eH}_{c} \mathrm{e}-\mathrm{Mod}$ ) on the triangulated category $D^{b}\left(\mathrm{eH}_{c} \mathrm{e}-\mathrm{Mod}\right)$ via the equivalence $\mathcal{L} o c$.

We have the following result.

Proposition 7.5.2. Fix a nonnegative $c \in \mathbb{Q}^{\text {good }}$ and let $p$ be large enough. Then, the functor $S_{+}$respects the above defined $t$-structures on $D^{b}\left(\mathcal{A}_{c}\right.$-Mod) and $D^{b}\left(\mathcal{A}_{c+1}\right.$-Mod).

Proof. We introduce another functor

$$
S_{-}: D^{b}\left(\mathcal{A}_{c+1^{-}} \text {Mod }\right) \longrightarrow D^{b}\left(\mathcal{A}_{c^{-}} \operatorname{Mod}\right), \quad \mathcal{M} \longmapsto S_{-}(\mathcal{M})={ }_{c} \mathcal{A}_{c+1} \otimes_{\mathcal{A}_{c+1}} \mathcal{M} .
$$

It is clear that $\left(S_{+}, S_{-}\right)$is a pair of adjoint functors which are triangulated equivalences quasi-inverse to each other.

Now, let $\mathrm{Ab}_{c}$ be the core of the $t$-structure on $D^{b}\left(\mathcal{A}_{c^{-}}\right.$Mod) transported from the natural $t$-structure on the category $D^{b}\left(\mathrm{eH}_{c} \mathrm{e}-\mathrm{Mod}\right)$, that is, the image of the abelian category $\mathrm{eH}_{c} \mathrm{e}-\mathrm{Mod}$ via the equivalence $\mathcal{L} o c$. By definition, we have

$$
\mathcal{M} \in \mathrm{Ab}_{c} \quad \Longleftrightarrow \quad H^{i}\left(\operatorname{Hilb}^{(1)}, \mathcal{M}\right)=0, \quad \forall i \neq 0 .
$$

Proving the proposition amounts to showing that the shift functor $S_{+}$takes the abelian category $A b_{c}$ to $A b_{c+1}$, resp. the functor $S_{-}$takes $A b_{c}$ to $A b_{c-1}$ if $c \geq 1$.

To prove this, observe first that it is enough to show that each of the two functors $S_{ \pm}$is right-exact with respect to the above $t$-structures. Once this is proved, it follows by adjunction between the two functors, that these functors are also leftexact, hence exact, with respect to the $t$-structures. To prove right-exactness, it suffices to prove that $S_{ \pm}(\mathcal{M}) \in \mathrm{Ab}_{c \pm 1}$ for any projective object $\mathcal{M} \in \mathrm{Ab}_{c}$. Any indecomposable projective in the abelian category $A b_{c}$ is by definition a direct summand of the free module $\mathcal{A}_{c}$. We have $S_{+}\left(\mathcal{A}_{c}\right)={ }_{c+1} \mathcal{A}_{c} \otimes_{\mathcal{A}_{c}} \mathcal{A}_{c}={ }_{c+1} \mathcal{A}_{c}$. Thus, we see from (7.5.3) that we have reduced the proof of the proposition to the following cohomology vanishing result:

$$
H^{i}\left(\operatorname{Hilb}^{(1)},{ }_{c+1} \mathcal{A}_{c}\right)=0, \quad\left[\text { resp. } \quad H^{i}\left(\operatorname{Hilb}^{(1)},{ }_{c} \mathcal{A}_{c+1}\right)=0, \quad \text { if } c \geq 1\right], \quad \forall i>0 .
$$

This cohomology vanishing is proved analogously to the proof of a similar cohomology vanishing in Proposition 4.3.3(iii). Specifically, let $\mathcal{O}(1)$ denote the standard ample line bundle on Hilb ${ }^{n} \mathbb{A}^{2}$. Then, one shows using the Rees algebra construction as in subsection 4.3, that the vector bundle ${ }_{c+1} \mathcal{A}_{c}$ on $\mathrm{Hilb}^{(1)}$ is a deformation of the sheaf $\operatorname{Fr}_{*} \mathcal{O}(1)$, resp. the vector bundle ${ }_{c} \mathcal{A}_{c+1}$ is a deformation of the sheaf 
$\mathrm{Fr}_{*} \mathrm{O}(-1)$. Therefore, the same semicontinuity argument as the one exploited in the proof of Proposition 4.3.3 would yield (7.5.4), provided we know that for all $i>0$ one has $H^{i}\left(\mathrm{Hilb}^{(1)}, \mathrm{Fr}_{*} \mathcal{O}(1)\right)=0$, resp. $H^{i}\left(\mathrm{Hilb}^{(1)}, \mathrm{Fr}_{*} \mathcal{O}(-1)\right)=0$. Clearly, we have $H^{i}\left(\mathrm{Hilb}^{(1)}, \mathrm{Fr}_{*} \mathcal{O}( \pm 1)\right)=H^{i}\left(\mathrm{Hilb}^{n} \mathbb{A}^{2}, \mathcal{O}( \pm 1)\right)$. The group on the RHS is known to vanish for all $i>0$ in the case of the line bundle $\mathcal{O}(1)$. To prove 3 a similar statement for $\mathcal{O}(-1)$ let $B=\mathbb{k}[\mathfrak{h} \times \mathfrak{h}]^{W}$, and let $F:=\Gamma\left(\operatorname{Hilb}^{n} \mathbb{A}^{2}, \mathcal{O}( \pm 1)\right)$. The space $F$ is a $B$-module which is known (see [H] or Appendix to [GG2]) to be isomorphic to the space of alternating polynomials on $\mathfrak{h} \times \mathfrak{h}$, viewed as a $\mathbb{k}[\mathfrak{h} \times \mathfrak{h}]^{W_{\text {- }}}$ module. Furthermore, according to Haiman, $F$ is a Cohen-Macaulay $B$-module. It follows that $R^{i} \operatorname{Hom}_{B}(F, B)=0$ for all $i \neq 0$.

On the other hand, in the derived category, there is the Grothendieck duality functor $\mathcal{M} \longmapsto \mathcal{M}^{\vee}$ which commutes with proper direct images. Applying this to the Hilbert-Chow morphism $\Upsilon: \operatorname{Hilb}^{n} \mathbb{A}^{2} \rightarrow(\mathfrak{h} \times \mathfrak{h}) / W$, we get

$$
\begin{aligned}
\mathrm{R} \Gamma\left(\operatorname{Hilb}^{n} \mathbb{A}^{2}, \mathcal{O}(-1)\right) & =\mathrm{R} \Gamma\left(\operatorname{Hilb}^{n} \mathbb{A}^{2}, \mathcal{O}(1)^{\vee}\right)=\mathrm{R} \Gamma\left((\mathfrak{h} \times \mathfrak{h}) / W, \Upsilon_{*} \mathcal{O}(1)^{\vee}\right) \\
& =\left(\operatorname{R} \Gamma((\mathfrak{h} \times \mathfrak{h}) / W, \mathcal{O}(1))^{\vee}=\operatorname{RHom}_{B}(F, B) .\right.
\end{aligned}
$$

We conclude that for all $i>0$ one has $H^{i}\left(\mathrm{Hilb}^{(1)}, \operatorname{Fr}_{*} \mathcal{O}(-1)\right)=0$, and the proposition is proved.

We now restrict our considerations to $\operatorname{Hilb}_{0}^{(1)} \subset \mathrm{Hilb}^{(1)}$, the formal neighborhood of the zero fiber of the Hilbert-Chow map. Let ${ }_{c+1} \widehat{\mathcal{A}}_{c}$ be the corresponding completion, an $\widehat{\mathcal{A}}_{c+1}-\widehat{\mathcal{A}}_{c}$-bimodule. There is an obvious analogue of diagram (7.5.1) and of Proposition 7.5 .2 where the scheme $\mathrm{Hilb}^{(1)}$ is replaced by Hilb ${ }_{0}^{(1)}$.

According to Theorem 7.4.1 our Azumaya algebras split on Hilb ${ }_{0}^{(1)}$. We write $\widehat{\mathcal{O}}:=\mathcal{O}_{{\widehat{\operatorname{Hib}_{0}}}_{0}^{(1)}}$ and let $\mathcal{W}_{c}^{*}=\mathcal{H o m}_{\widehat{\mathcal{O}}}\left(\mathcal{W}_{c}, \widehat{\mathcal{O}}\right)$ be the dual of the splitting bundle $\mathcal{W}_{c}$. Thus, $\mathcal{W}_{c}^{*}$ is a right $\widehat{\mathcal{A}}_{c}$-module and we have $\widehat{\mathcal{A}}_{c}=\mathcal{W}_{c} \otimes_{\widehat{\mathcal{O}}} \mathcal{W}_{c}^{*}$. Thus, we have an equivalence

$$
D^{b}\left(\operatorname{Coh}\left(\widehat{\operatorname{Hilb}}_{0}^{(1)}\right)\right) \stackrel{\sim}{\longrightarrow} D^{b}\left(\mathcal{A}_{c^{-}} \operatorname{Mod}\right), \quad \mathcal{F} \longmapsto \mathcal{W}_{c} \otimes_{\widehat{\mathcal{O}}} \mathcal{F}
$$

with quasi-inverse functor $\mathcal{M} \longmapsto \mathcal{W}_{c}^{*} \otimes_{\widehat{\mathcal{A}}_{c}} \mathcal{M}$.

Let $\mathcal{C}_{c}$ be the the abelian subcategory in $D^{b}\left(\operatorname{Coh}\left(\widehat{\operatorname{Hilb}}_{0}^{(1)}\right)\right)$ corresponding to the abelian category $\mathrm{Ab}_{c}$ under the equivalence (7.5.5). Thus, the abelian category $\mathrm{C}_{c}$ is the core of an "exotic" $t$-structure on $D^{b}\left(\operatorname{Coh}\left(\widehat{\operatorname{Hilb}}_{0}^{(1)}\right)\right)$. By definition, we have

$$
\mathcal{F} \in \mathcal{C}_{c} \quad \Longleftrightarrow \quad H^{i}\left(\operatorname{Hilb}^{(1)}, \mathcal{W}_{c} \otimes_{\widehat{\mathcal{O}}} \mathcal{F}\right)=0, \quad \forall i \neq 0 .
$$

Furthermore, we may transport the geometric shift functor $S_{+}$via the equivalence (7.5.5) to obtain the following functor:

$$
\begin{gathered}
\mathbb{S}_{+}: D^{b}\left(\operatorname{Coh}\left(\widehat{\operatorname{Hilb}}_{0}^{(1)}\right)\right) \rightarrow D^{b}\left(\operatorname{Coh}\left(\widehat{\operatorname{Hilb}}_{0}^{(1)}\right)\right), \\
\mathbb{S}_{+}(\mathcal{F})=\mathcal{W}_{c+1}^{*} \otimes{\widehat{\mathcal{A}_{c+1}}}_{c+1} \widehat{\mathcal{A}}_{c} \otimes_{\widehat{\mathcal{A}_{c}}} \mathcal{W}_{c} \otimes_{\widehat{\mathcal{O}}} \mathcal{F} .
\end{gathered}
$$

Note that, in the above formula, one may choose the splitting bundle $\mathcal{W}_{c+1}$ to be $\mathcal{W}_{c+1}:=S_{+}\left(\mathcal{W}_{c}\right)$. With this choice, from Proposition 7.5.2, we obtain:

Corollary 7.5.6. For any nonnegative $c \in \mathbb{Q}^{\text {good }}$, the functor $\mathbb{S}_{+}$restricts to an equivalence $\mathcal{C}_{c} \stackrel{\sim}{\longrightarrow} \mathcal{C}_{c+1}$, of abelian categories.

\footnotetext{
${ }^{3}$ The short argument below was suggested to us by M. Brion.
} 


\section{INDUCTION FUNCTOR AND COMPARISON WITH EG}

In this subsection, we let $\mathbb{k}$ be an arbitrary algebraically closed field, either of characteristic zero or of characteristic $p$.

8.1. Let $P$ be a linear algebraic group with Lie algebra $\mathfrak{p}$, and $\mathrm{D}$ an associative algebra equipped with a $P$-action by algebra automorphisms and with a $P$-equivariant algebra map $\rho: \mathcal{U} \mathfrak{p} \rightarrow \mathrm{D}$, as in subsection 3.4. Recall our convention to write $\mathrm{D} \cdot J$ instead of $\mathrm{D} \cdot \rho(J)$.

Definition 8.1.1. Given a two-sided ideal $J \subset \mathcal{U} \mathfrak{p}$, let $\operatorname{Ind}(\mathrm{D} \uparrow J) \subset \mathrm{D}$ denote the annihilator of the left D-module D/D.$J$. This is a two-sided ideal in D, called the ideal induced from $J$.

It follows from the definition that $\operatorname{Ind}(\mathrm{D} \uparrow J)$ is the maximal two-sided ideal of $\mathrm{D}$ contained in the left ideal D. $J$.

Assume next that $P$ is an algebraic subgroup in another connected linear algebraic group $G$. Set $\mathfrak{g}:=\operatorname{Lie} G$, and let $\mathcal{U} \mathfrak{g}$ be the corresponding enveloping algebra. Thus, $\mathfrak{p} \subset \mathfrak{g}$ and $\mathcal{U} \mathfrak{p} \subset \mathcal{U} \mathfrak{g}$. Given a two-sided ideal $J \subset \mathcal{U} \mathfrak{p}$, as above, we may form an induced ideal $\operatorname{Ind}(\mathcal{U} \mathfrak{g} \uparrow J) \subset \mathcal{U} \mathfrak{g}$.

Now, let $G$ act on an associative algebra $\mathrm{D}$, and let $\mathcal{U} \mathfrak{g} \rightarrow \mathrm{D}$ be a $G$-equivariant algebra map. We consider the composite map $\mathcal{U} \mathfrak{p} \hookrightarrow \mathcal{U} \mathfrak{g} \rightarrow \mathrm{D}$. Let $J \subset \mathcal{U} \mathfrak{p}$ be a two-sided ideal and $\operatorname{Ind}(\mathcal{U} \mathfrak{g} \uparrow J) \subset \mathcal{U} \mathfrak{g}$ the corresponding induced ideal. Since $J \subset \mathcal{U} \mathfrak{g} \cdot J$, we have $\mathrm{D} \cdot \operatorname{Ind}(\mathcal{U} \mathfrak{g} \uparrow J) \subset \mathrm{D} \cdot J$. Hence, the projection D/D.Ind $(\mathcal{U} \mathfrak{g} \uparrow J) \rightarrow$ $\mathrm{D} / \mathrm{D} \cdot J$ induces an algebra map:

$$
(\mathrm{D} / \mathrm{D} \cdot \operatorname{Ind}(\mathcal{U} \mathfrak{g} \uparrow J))^{G} \longrightarrow(\mathrm{D} / \mathrm{D} \cdot J)^{P} .
$$

8.2. We recall the setup of subsection 6.1, so $V$ is an $n$-dimensional vector space over $\mathbb{k}$, and we put $G=\operatorname{GL}(V)$ and $\mathfrak{g}=\mathfrak{g l}(V)$. We also fix $c \in \mathbb{k}$ and let $\chi_{c}:=$ $c \cdot \operatorname{tr}: \mathfrak{g} \rightarrow \mathbb{k}$ be the corresponding Lie algebra character.

From now on, we fix a nonzero vector $\mathbf{v} \in V$. Let $P$ be a parabolic subgroup of $G$ formed by the maps $V \rightarrow V$ that preserve the line $\mathbb{k} \mathbf{v}$. Thus $G / P \cong \mathbb{P}(V)$, the $(n-1)$-dimensional projective space associated to $V$. We put $\mathfrak{p}:=$ Lie $P$, and write $\chi_{c}^{\mathfrak{p}}:=\left.\chi_{c}\right|_{\mathfrak{p}}$ for the character $\chi_{c}$ restricted to the subalgebra $\mathfrak{p} \subset \mathfrak{g}$. Thus $\chi_{c}^{\mathfrak{p}} \in \mathfrak{p}^{*}$ is a $P$-fixed point for the coadjoint action of $P$ on $\mathfrak{p}^{*}$.

We extend $\chi_{c}^{\mathfrak{p}}$ to an associative algebra homomorphism $\chi_{c}^{\mathfrak{p}}: \mathcal{U} \mathfrak{p} \rightarrow \mathbb{k}$, and let $J_{c}:=\operatorname{Ker}(\mathcal{U} \mathfrak{p} \rightarrow \mathbb{k})$, denote the corresponding two-sided ideal generated by the elements $\{x-c \cdot \operatorname{tr}(x)\}_{x \in \mathfrak{p}}$; see Definition 3.3.1. Also, write $\operatorname{Ind}_{c}:=\operatorname{Ind}\left(\mathcal{U} \mathfrak{g} \uparrow J_{c}\right)$ for the two-sided ideal in $\mathcal{U} \mathfrak{g}$ induced from $J_{c}$. It is known that $\operatorname{lnd}_{c}$ is a primitive ideal in $\mathcal{U} \mathfrak{g}$, moreover, it is exactly the primitive ideal considered in [EG].

The adjoint action of $G$ on $\mathfrak{g}$ gives rise to an associative algebra homomorphism ad $: \mathcal{U} \mathfrak{g} \rightarrow \mathcal{D}(\mathfrak{g})$. Thus, we may consider the homomorphism (8.1.2) in the special case $\mathrm{D}:=\mathcal{D}(\mathfrak{g}), J:=J_{c}$. As usual, we abuse the notation and write $\mathcal{D}(\mathfrak{g}) \cdot \operatorname{Ind}_{c}$ instead of $\mathcal{D}(\mathfrak{g}) \cdot \operatorname{ad} \operatorname{lnd}_{c}$ for the corresponding left ideal in $\mathcal{D}(\mathfrak{g})$.

We propose the following:

Conjecture 8.2.1. For any $c \in \mathbb{k}$, the following canonical map is an algebra isomorphism:

$$
\left(\mathcal{D}(\mathfrak{g}) / \mathcal{D}(\mathfrak{g}) \cdot \operatorname{lnd}_{c}\right)^{G} \stackrel{(8.1 .2)}{\longrightarrow}\left(\mathcal{D}(\mathfrak{g}) / \mathcal{D}(\mathfrak{g}) \cdot J_{c}\right)^{P}
$$


8.3. In chapter 7 of [EG], the authors have constructed, for any $c \in \mathbb{k}$, an algebra homomorphism, called the deformed Harish-Chandra homomorphism:

$$
\Phi_{c}:\left(\mathcal{D}\left(\mathfrak{g}^{\mathrm{rs}}\right) / \mathcal{D}\left(\mathfrak{g}^{\mathrm{rs}}\right) \cdot \operatorname{lnd}_{c}\right)^{G} \longrightarrow \mathcal{D}\left(\mathfrak{h}^{\mathrm{reg}}\right)^{W}
$$

such that one has $\Phi_{c}\left(\Delta_{\mathfrak{g}}\right)=\mathrm{L}_{c}$, the Calogero-Moser operator.

The reader should be warned that the map referred to as the deformed HarishChandra homomorphism in EG] was actually a map $\mathcal{D}\left(\mathfrak{g}^{\text {rs }}\right)^{G} \longrightarrow \mathcal{D}\left(\mathfrak{h}^{\text {reg }}\right)^{W}$. However, it has been shown in [EG] that the latter map vanishes on the two-sided ideal

$$
\left(\mathcal{D}\left(\mathfrak{g}^{\mathrm{rs}}\right) \cdot \operatorname{lnd}_{c}\right)^{G}=\left(\mathcal{D}\left(\mathfrak{g}^{\mathrm{rs}}\right) \cdot \operatorname{Ind}_{c}\right) \cap \mathcal{D}\left(\mathfrak{g}^{\mathrm{rs}}\right)^{G},
$$

hence, descends to a well-defined homomorphism

$$
\left(\mathcal{D}\left(\mathfrak{g}^{\mathrm{rs}}\right) / \mathcal{D}\left(\mathfrak{g}^{\mathrm{rs}}\right) \cdot \operatorname{lnd}_{c}\right)^{G}=\mathcal{D}\left(\mathfrak{g}^{\mathrm{rs}}\right)^{G} /\left(\mathcal{D}\left(\mathfrak{g}^{\mathrm{rs}}\right) \cdot \operatorname{lnd}_{c}\right)^{G} \longrightarrow \mathcal{D}\left(\mathfrak{h}^{\mathrm{reg}}\right)^{W}
$$

where the equality on the left exploits semisimplicity of the ad $G$-action on $\mathcal{D}\left(\mathfrak{g}^{\mathrm{rs}}\right)$.

The construction of EG can also be carried out over a field $\mathbb{k}$ of characteristic $p$. In that case, the adjoint $G$-action on $\mathcal{D}\left(\mathfrak{g}^{\mathrm{rs}}\right)$ is not semisimple, so the equality on the left of (8.3.2) does not hold, in general. A more careful analysis of the construction of [EG], similar to that of subsection 5.3 of the present paper, shows that it actually produces, without any semisimplicity assumption, a homomorphism of the form (8.3.1).

8.4. Recall the open subset $U \subset \mathfrak{g}^{\mathrm{rs}} \times V^{\circ}$ formed by all pairs $(x, v)$ such that $v$ is a cyclic vector for $x$; see Definition 5.3.1, Let $\mathfrak{g}^{\circ} \subset \mathfrak{g}^{\text {rs }}$ be the set of all elements $x \in \mathfrak{g}=\mathfrak{g l}(V)$ such that our fixed vector $\mathbf{v} \in V$ is a cyclic vector for $x$. Clearly, $\mathfrak{g}^{\circ}$ is an Ad $P$-stable Zariski open dense subset of $\mathfrak{g}$. Recall also the two-sided ideal $I_{c} \subset \mathcal{U g}$ introduced in subsection 5.3 , and observe that $\operatorname{Ind}_{c} \subset I_{c}$.

The following result provides a relation between the Harish-Chandra homomorphism $\Psi_{c}$ of Proposition 5.4.1, and the homomorphism (8.3.1).

Proposition 8.4.1. There is a natural algebra map $F_{c}:\left(\mathcal{D}\left(\mathfrak{g}^{\circ}\right) / \mathcal{D}\left(\mathfrak{g}^{\circ}\right) \cdot J_{c}\right)^{P} \longrightarrow$ $\Gamma\left(U, \mathcal{D}_{U} / \mathcal{D}_{U} \cdot I_{c}\right)^{G}$ making the following diagram commute:

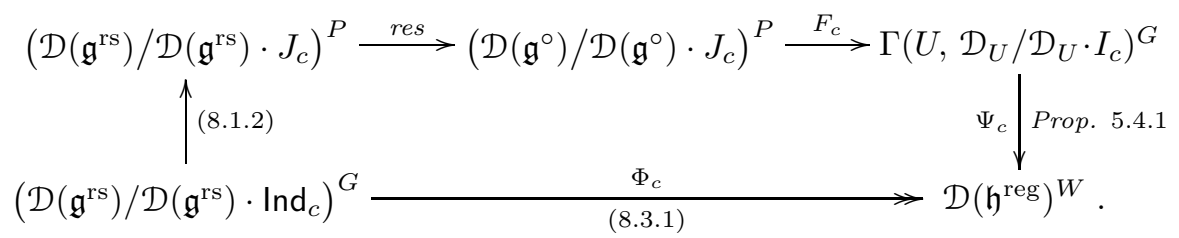

Sketch of Proof. The assignment $(x, v) \longmapsto \mathbb{k} v$ clearly gives a $G$-equivariant fibration $f: U \longrightarrow \mathbb{P}(V)$. The fiber of this map over the class of the line $\mathbb{k} \mathbf{v} \in \mathbb{P}(V)$ is the set $U_{\mathbf{v}}=\left\{(x, t \cdot \mathbf{v}) \mid x \in \mathfrak{g}^{\circ}, t \in \mathbb{k}^{\times}\right\}$. Thus, we have a $G$-equivariant isomorphism $U \cong G \times{ }_{P} U_{\mathbf{v}}$. Furthermore, it is clear that the map $(x, t) \mapsto(x, t \cdot \mathbf{v})$ gives a $P$-equivariant isomorphism $\mathfrak{g}^{\circ} \times \mathbb{G}_{\mathbf{m}} \stackrel{\sim}{\longrightarrow} U_{\mathbf{v}}$. Thus, we obtain the following diagram:

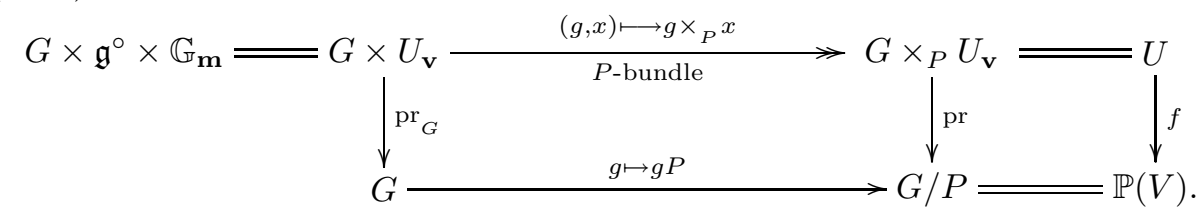


Let $X:=G \times U_{\mathbf{v}} \cong G \times \mathfrak{g}^{\circ} \times \mathbb{G}_{\mathbf{m}}$. The assignment $u \longmapsto 1 \otimes u \otimes 1$ gives an algebra map

$$
\mathcal{D}\left(\mathfrak{g}^{\circ}\right) \longrightarrow \mathcal{D}(G) \otimes \mathcal{D}\left(\mathfrak{g}^{\circ}\right) \otimes \mathcal{D}\left(\mathbb{G}_{\mathbf{m}}\right) \stackrel{\sim}{\longrightarrow} \mathcal{D}\left(G \times U_{\mathbf{v}}\right)=\mathcal{D}(X) .
$$

We compose this map with isomorphism (3.7.2) applied to $X, Y:=G \times{ }_{P} U_{\mathbf{v}}$, and to the $P$-bundle map in the top row of diagram (8.4.2). This way, we get a chain of algebra morphisms:

$$
F_{c}^{\prime}: \mathcal{D}\left(\mathfrak{g}^{\circ}\right)^{P} \longrightarrow \mathcal{D}(X)^{P} \longrightarrow \mathcal{D}\left(X \mathcal{D}_{X} / \mathcal{D}_{X} \cdot J_{c}\right)^{P} \longrightarrow \mathcal{D}\left(U, \chi_{c}\right) .
$$

One can show, using the equality $\operatorname{Ind}\left(\mathcal{U} \mathfrak{g} \uparrow J_{c}\right)=\bigcap_{g \in G} \operatorname{Ad} g\left(\mathcal{U} \mathfrak{g} \cdot J_{c}\right)$, that a suitably refined version of the above construction produces a well-defined algebra map

$$
F_{c}^{\prime \prime}: \Gamma\left(\mathfrak{g}^{\circ}, \mathcal{D}_{\mathfrak{g}^{\circ}} / \mathcal{D}_{\mathfrak{g}^{\circ}} \cdot J_{c}\right)^{P} \longrightarrow \Gamma\left(U, \mathcal{D}_{U} / \mathcal{D}_{U} \cdot \operatorname{lnd}_{c}\right)^{G} .
$$

Furthermore, one verifies that composing the map $F_{c}^{\prime \prime}$ with the natural restriction

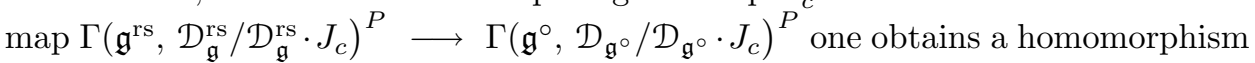
$F_{c}$ that makes the diagram of the proposition commute.

\section{Appendix: The $p$-CEnter of Symplectic RefleCtion algebras}

\section{by Pavel Etingof}

9.1. Let $\mathbb{k}$ be an algebraically closed field of characteristic $p>0, V$ a finitedimensional symplectic vector space over $\mathbb{k}$, and $\Gamma \subset S p(V)$ a finite subgroup. We assume that $p$ is odd and prime to $|\Gamma|$.

Write $S \subset \Gamma$ for the set of symplectic reflections in $\Gamma$. Let $\mathrm{H}_{t, c}(V, \Gamma)$ be the symplectic reflection algebra with parameters $t$ and $c \in \mathbb{k}[S]^{\Gamma}$, as defined in [EG]. For $t=c=0$, we have $\mathrm{H}_{0,0} \simeq S V \# \Gamma$, a cross-product of $S V$ with the group $\Gamma$, to be denoted $\overline{\mathrm{H}}$.

In general, the algebra $\mathrm{H}_{t, c}$ comes equipped with an increasing filtration such that gr $_{t, c} \simeq \overline{\mathrm{H}}=S V \# \Gamma$. Let $\mathrm{Z}_{t, c}$ be the center of $\mathrm{H}_{t, c}$, equipped with the induced filtration.

The main result of this appendix is the following theorem.

Theorem 9.1.1. There is a graded algebra isomorphism $\operatorname{gr}\left(\mathrm{Z}_{1, c}\right)=\left((S V)^{p}\right)^{\Gamma}$.

Corollary 9.1.2 (Satake isomorphism). The map $\mathrm{Z}_{1, c} \rightarrow \mathrm{eH}_{c} \mathrm{e}, z \longmapsto \mathrm{e} \cdot z$ gives an isomorphism between the centers of the algebras $\mathrm{H}_{1, c}$ and $\mathrm{eH}_{1, c} \mathrm{e}$, respectively.

Proof of Corollary 9.1.2. The associated graded map is an isomorphism.

Remark 9.1.3. One may consider the field $\mathbb{k}$ as the residue class field in $W(\mathbb{k})$, the ring of Witt vectors of $\mathbb{k}$. The algebra $\mathrm{H}_{1, c}$ may thus be regarded as a specialization of a $W(\mathbb{k})$-algebra. Applying the well-known (see $\mathrm{Ha}$ ) Hayashi construction to this situation one obtains a natural Poisson bracket on the $p$-center $\mathrm{Z}_{1, c} \subset \mathrm{H}_{1, c}$.

9.2. To prove Theorem 9.1.1 we need the following result.

Proposition 9.2.1. The Hochschild cohomology of the algebra $\overline{\mathrm{H}}$ is given by the formula

$$
H H^{m}(\overline{\mathrm{H}})=\left(\bigoplus_{g \in \Gamma: \operatorname{codim} V^{g} \leq m} \Omega^{m-\operatorname{codim} V^{g}}\left(V^{g}\right)\right)^{\Gamma} .
$$


(here $\Omega^{j}$ denotes the space of differential forms of degree $j$ ) Moreover, elements $z \in \mathrm{Z}_{0,0}=H H^{0}(\overline{\mathrm{H}})$ act on this cohomology by multiplying differential forms on $V^{g}$ by the restriction of $z$ to $V^{g}$.

Proof. The first statement is straightforward by using Koszul resolutions, similarly to AFLS. The second statement is easy.

9.3. Proof of Theorem 9.1.1. Since gr $_{t, c}=\overline{\mathrm{H}}$, we have the Brylinski spectral sequence $[\mathrm{Br}$. This spectral sequence has

$$
E_{1}^{r, q}=H H^{r+q}(\overline{\mathrm{H}}), \quad \text { and } \quad E_{\infty}^{r, q}=\operatorname{gr}\left(H H^{r+q}\left(\mathrm{H}_{t, c}\right)\right) .
$$

Since the algebra $\mathrm{H}_{t, c}$ is $\mathbb{Z} / 2 \mathbb{Z}$-graded, the differentials $d_{j}$ with odd subscripts $j$ in this spectral sequence vanish automatically. Therefore, we will abuse notation by writing $d_{i}, E_{i}^{r, q}$ instead of $d_{2 i}, E_{2 i}^{r, q}$.

Let us now consider the differential $d_{1}$. Obviously, we have $d_{1}=t d_{1}^{(1)}+$ $\sum_{s \in S / \Gamma} c_{s} d_{1}^{(s)}$, where $d_{1}^{(1)}$ is the differential corresponding to $c=0, t=1$. It was checked by Brylinski that $d_{1}^{(1)}$ is the De Rham differential.

By a result of [EG] (which generalizes in a straightforward manner to positive characteristic), we have gr $Z_{0, c}=Z_{0,0}$. Thus, the differentials $d_{1}^{(s)}$ are zero in cohomological degree zero, so they are morphisms of modules over $Z_{0,0}$. Thus, in cohomological degree 1 , these differentials must land in twisted (torsion) components (as generically on $M_{c}:=\operatorname{Spec}\left(Z_{0, c}\right)$ the algebra $\mathrm{H}_{0, c}$ is Azumaya). This implies that $E_{2}^{-q, q}=\left((S V)^{p}\right)^{\Gamma}$, and $E_{2}^{-q+1, q}$ is a submodule (over $\left((S V)^{p}\right)^{\Gamma}$ ) of $\Omega_{(p)}^{1}(V)^{\Gamma}$, where $\Omega_{(p)}^{1}(V)$ denotes the first cohomology of the De Rham complex of $V$.

We will now show that all the higher differentials $d_{m}, m \geq 2$, vanish in cohomological degree zero, and hence $E_{\infty}^{-q, q}=\left((S V)^{p}\right)^{\Gamma}$, as desired. Assume the contrary, and let $m \geq 2$ be the smallest number such that $d_{m}$ does not vanish in cohomological degree zero. Then we can view $d_{m}$ as a derivation $d_{m}:\left((S V)^{p}\right)^{\Gamma} \rightarrow \Omega_{(p)}^{1}(V)^{\Gamma}$.

Recall now $([\underline{K}$, Theorem 7.2) that if $X$ is a smooth affine variety over $\mathbb{k}$, then the cohomology of the De Rham complex of $X$ twisted by the Frobenius map can be identified with the module of differential forms on $X$ (as a graded $\mathcal{O}_{X}$-module) via a map $C^{-1}: \Omega^{\bullet}(X) \rightarrow H^{\bullet}\left(\operatorname{Fr}_{*} \Omega^{\bullet}(X)\right)$, called the Cartier operator. This map is defined by the formulas $C^{-1}(a)=a^{p}, C^{-1}(d a)=a^{p-1} d a$ for functions $a$ on $X$.

Let $d_{m}^{\prime}=C d_{m} C^{-1}$. Then $d_{m}^{\prime}:(S V)^{\Gamma} \rightarrow \Omega^{1}(V)^{\Gamma}$ is a derivation. Thus, $d_{m}^{\prime}$ gives rise to a $\Gamma$-equivariant regular function $f$ on $V_{\text {reg }}$ with values in $V \otimes V^{*}$, where $V_{\text {reg }}$ is the set of points of $V$ which have the trivial stabilizer in $\Gamma$. Since the complement of $V_{\text {reg }}$ has codimension 2, the function $f$ extends to $V$ and defines an element of $S V \otimes V \otimes V^{*}$. Hence $d_{m}^{\prime}$ is obtained by restricting a map $d_{m}^{\prime}: S V \rightarrow \Omega^{1}(V)$ to $\Gamma$-invariants. Therefore, $d_{m}$ is obtained by restricting a map $d_{m}:(S V)^{p} \rightarrow \Omega_{(p)}^{1}(V)$ to $\Gamma$-invariants.

Now observe that the map $d_{m}$ must have degree $-2 m+2<0$. On the other hand, the generators of $(S V)^{p}$ sit in degree $p$, while the lowest degree in $\Omega_{(p)}^{1}(V)$ is also equal to $p$. This means that $d_{m}=0$, which is a contradiction. The theorem is proved. 


\section{REFERENCES}

[AFLS] J. Alev, M.A. Farinati, T. Lambre, and A.L. Solotar, Homologie des invariants d'une algèbre de Weyl sous l'action d'un groupe fini. J. of Algebra 232 (2000), 564-577. MR 1792746 (2002c:16047)

[BB] A. Beilinson, J. Bernstein, A proof of Jantzen conjectures. I. M. Gelfand Seminar, 1-50, Adv. Soviet Math., 16, Part 1, Amer. Math. Soc., Providence, RI, 1993. MR1237825 (95a:22022)

[BGS] A. Beilinson, V. Ginzburg, W. Soergel, Koszul duality patterns in representation theory, J. Amer. Math. Soc. 9 (1996), 473-527. MR1322847 (96k:17010)

[BEG] Yu. Berest, P. Etingof, V. Ginzburg, Cherednik algebras and differential operators on quasi-invariants. Duke Math. J. 118 (2003), 279-337. MR.1980996 (2004f:16039)

[BEG2] Yu. Berest, P. Etingof, V. Ginzburg, Finite-dimensional representations of rational Cherednik algebras. Int. Math. Res. Not. 2003, no. 19, 1053-1088. MR1961261 (2004h:16027)

[BK] R. Bezrukavnikov, D. Kaledin, MacKay equivalence for symplectic quotient resolutions of singularities, Proc. of the Steklov Inst. of Math. 246 (2004), 13-33. MR.2101282

[BMR] R. Bezrukavnikov, I. Mirković, D. Rumynin, Localization of modules for a semisimple Lie algebra in prime characteristic, Ann. Math. (2006). [arXiv:math.RT/0205144].

[BKR] T. Bridgeland, A. King, M. Reid, The McKay correspondence as an equivalence of derived categories. J. Amer. Math. Soc. 14 (2001), 535-554. MR1824990 (2002f:14023)

[Br] J.-L. Brylinski, A differential complex for Poisson manifolds. J. Diff. Geom. 28 (1988), 93-114. MR0950556 (89m:58006)

[CG] N. Chriss, V. Ginzburg, Representation theory and complex geometry. Birkhäuser Boston, 1997. MR 1433132 (98i:22021)

[Ch] I. Cherednik, Double affine Hecke algebras, Knizhnik-Zamolodchikov equations, and Macdonald operators, IMRN (Duke Math. J.) 9 (1992), 171-180. MR.1185831 (94b:17040)

[DO] C. Dunkl, E. Opdam, Dunkl operators for complex reflection groups. Proc. London Math. Soc. 86 (2003), 70-108. MR1971464 (2004d:20040)

[EG] P. Etingof, V. Ginzburg, Symplectic reflection algebras, Calogero-Moser space, and deformed Harish-Chandra homomorphism. Invent. Math. 147 (2002), 243-348. MR1881922 (2003b:16021)

[FG] M. Finkelberg, V. Ginzburg, Character sheaves for Cherednik algebras. (in preparation).

[Ga] O. Gabber, Some theorems on Azumaya algebras. The Brauer group (Sem., Les Plans-surBex, 1980), pp. 129-209, Lecture Notes in Math., 844, Springer, Berlin-New York, 1981. MR0611868 (83d:13004)

[GIT] D. Mumford, J. Fogarty, F. Kirwan, Geometric invariant theory. Third edition. Ergebnisse der Mathematik und ihrer Grenzgebiete 34 Springer-Verlag, Berlin, 1994. MR1304906 (95m:14012)

[GG] W. L. Gan, V. Ginzburg, Quantization of Slodowy slices. Int. Math. Res. Not. 2002, no. 5, 243-255. MR1876934 (2002m:53129)

[GG2] W. L. Gan, V. Ginzburg, Almost-commuting variety, D-modules, and Cherednik Algebras, Int. Math. Res. Publ. (2006). arXiv:math.RT/0409262.

[GS] I. Gordon, T. Stafford, Rational Cherednik algebras and Hilbert schemes of points. Adv. in Math. (2006). [arXiv:math.RA/0407516].

[Gr] A. Grothendieck, SGA 1, Lecture Notes in Mathematics, 224 (1971). MR0354651 (50:7129)

[H] M. Haiman, Vanishing theorems and character formulas for the Hilbert scheme of points in the plane, Invent. Math. 149 (2002), 371-407. MR1918676 (2003f:14006)

[Har] R. Hartshorne, Algebraic geometry. Graduate Texts in Mathematics, 52. Springer-Verlag, New York-Heidelberg, 1977. MR0463157 (57:3116)

[Ha] T. Hayashi, Sugawara operators and Kac-Kazhdan conjecture. Invent. Math. 94 (1988), 13-52. MR0958588 (90c:17035)

[J] N. Jacobson, Lie algebras. Interscience Tracts in Pure and Applied Mathematics, 10 Interscience Publishers (a division of John Wiley \& Sons), New York-London 1962. MR0143793 (26:1345) 
[Ja] J.C. Jantzen, Representations of Lie algebras in prime characteristic. Notes by Iain Gordon. NATO Adv. Sci. Inst. Ser. C Math. Phys. Sci., 514, Representation theories and algebraic geometry (Montreal, PQ, 1997), 185-235, Kluwer Acad. Publ., Dordrecht, 1998. MR:1649627(99h:17026)

[K] N. Katz, Nilpotent connections and the monodromy theorem: Applications of a result of Turrittin Publ. Math.IHES 39(1970). MR0291177 (45:271)

[KKS] D. Kazhdan, B. Kostant, and S. Sternberg, Hamiltonian group actions and dynamical systems of Calogero type, Comm. Pure Appl. Math., 31(1978), 481-507. MR 478225|(57:7711)

[KT] S. Kumar, J. F. Thomsen, Frobenius splitting of Hilbert schemes of points on surfaces. Math. Ann. 319 (2001), 797-808. MR.1825408 (2002d:14004)

[La] F. Latour, Representations of rational Cherednik algebras of rank 1 in positive characteristic, J. Pure Appl. Algebra 195 (2005), 97-112. MR.2100312

[MVK] V. B. Mehta, W. van der Kallen, On a Grauert-Riemenschneider vanishing theorem for Frobenius split varieties in characteristic p. Invent. Math. 108 (1992), 11-13. MR1156382 (93a:14017)

[Na1] H. Nakajima, Lectures on Hilbert schemes of points on surfaces. University Lecture Series, 18, American Mathematical Society, Providence, RI, 1999. MR.1711344 (2001b:14007)

[Na2] H. Nakajima, Quiver varieties and Kac-Moody algebras. Duke Math. J. 91 (1998), 515560. MR.1604167 (99b:17033)

[Ob] A. Oblomkov, Double affine Hecke algebras and Calogero-Moser spaces. Represent. Theory 8 (2004), 243-266. MR2077482 (2005e:20005)

[Pr] A. Premet, Special transverse slices and their enveloping algebras. Adv. Math. 170 (2002), 1-55. MR 1929302 (2003k:17014)

[PS] A. Premet, S. Skryabin, Representations of restricted Lie algebras and families of associative L-algebras. J. Reine Angew. Math. 507 (1999), 189-218. MR1670211|(99m:17026)

[Q] D. Quillen, On the endormorphism ring of a simple module over an enveloping algebra. Proc. Amer. Math. Soc. 211969 171-172. MR0238892 (39:252)

[Wi] G. Wilson, Collisions of Calogero-Moser particles and an adelic Grassmannian, Inv. Math. 133 (1998), 1-41. MR.1626461 (99f:58107)

Department of Mathematics, M.I.T., 77 Massachusetts Ave, Cambridge, Massachusetts 02139

E-mail address: bezrukav@math.mit.edu

Independent University of Moscow, 11 Bolshoy Vlasyevskiy Per., 119002 Moscow, Russia

E-mail address: fnklberg@mccme.ru

Department of Mathematics, University of Chicago, Chicago, Illinois 60637

E-mail address: ginzburg@math.uchicago.edu

Department of Mathematics, M.I.T., 77 Massachusetts Ave, Cambridge, Massachusetts 02139

E-mail address: etingof@math.mit.edu 